\title{
Investigation of Orbital Debris Situational Awareness with Constellation Design and Evaluation
}

\author{
Ethan B. Ohriner \\ Thesis submitted to the Faculty of the \\ Virginia Polytechnic Institute and State University \\ in partial fulfillment of the requirements for the degree of
}

Masters of Science

In

Aerospace Engineering

Jonathan T. Black, Chair

Kevin Shinpaugh

Matt VanDyke

11 December 2020

Blacksburg, Virginia

Keywords: orbital debris, laser debris removal, constellation design, space situational awareness

Copyright 2020, Ethan B. Ohriner 


\title{
Investigation of Orbital Debris Situational Awareness with Constellation Design and Evaluation
}

\author{
Ethan B. Ohriner
}

\begin{abstract}
Orbital debris is a current and growing threat to reliable space operations and new space vehicle traffic. As space traffic increases, so does the economic impact of orbital debris on the sustainability of systems that increasingly support national security and international commerce. Much of the debris collision risk is concentrated in specific high-density debris clusters in key regions of Low Earth Orbit (LEO). A potential long-term solution is to employ a constellation of observation satellites within these debris clusters to improve monitoring and characterization efforts, and engage in Laser Debris Removal (LDR) as means of collision mitigation. Here we adapted and improved a previous methodology for evaluating such designs. Further, we performed an analysis on the observer constellations' effectiveness over a range of circular, elliptical, and self-maneuvering designs. Our results show that increasingly complex designs result in improved performance of various criteria and that the proposed method of observation could significantly reduce the threat orbital debris poses to space operations and economic growth.
\end{abstract}




\title{
Investigation of Orbital Debris Situational Awareness with Constellation Design and Evaluation
}

\author{
Ethan B. Ohriner
}

\section{GENERAL AUDIENCE ABSTRACT}

Orbital debris is defined as all non-operational, man-made objects currently in space. US national space regulations require every new satellite to have a de-orbit plan to prevent the creation of new debris, but fails to address the thousands of derelict objects currently hindering space operations. As space traffic increases, so does the economic impact of orbital debris on the sustainability of systems that increasingly support national security and commercial growth. While orbital debris is usually assessed by looking at the full volume of space, most massive debris objects are concentrated in high-density clusters with a higher than normal probability for collision. A potential solution to the growing orbital debris problem is to place a group of observation satellites within these debris clusters to both improve monitoring capabilities and provide a means for preventing potential collisions by engaging with debris via Laser Debris Removal (LDR). This research presents a methodology for comparing and contrasting different observer satellite constellation designs. Our results show that increasingly complex orbit designs improve various performance criteria, but ultimately orbits that more closely match those of the debris objects provide the best coverage. The proposed method of observation and engagement could significantly reduce the threat orbital debris poses to space operations and economic growth. 


\section{Table of Contents}

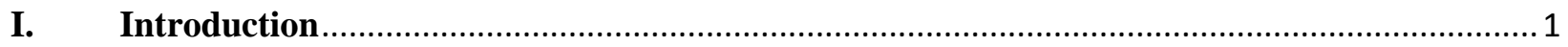

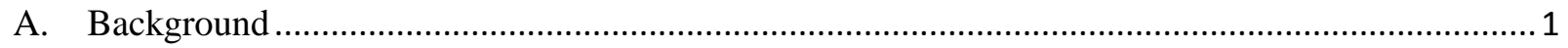

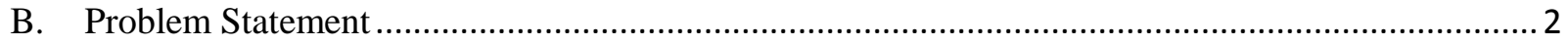

C. Research Objectives, Questions, and Hypothesis …................................................................. 4

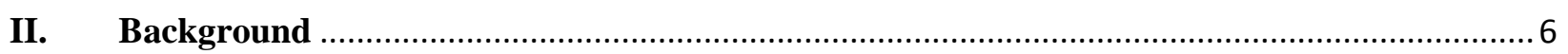

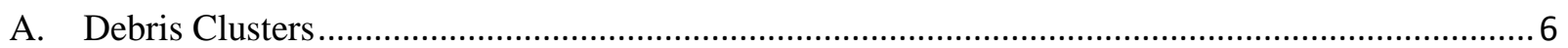

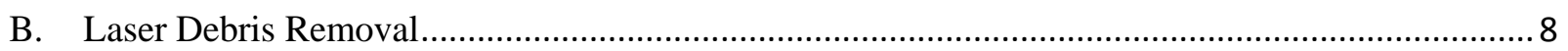

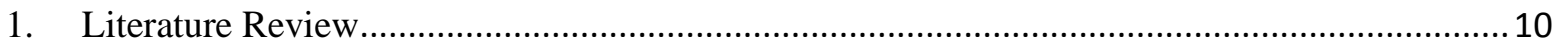

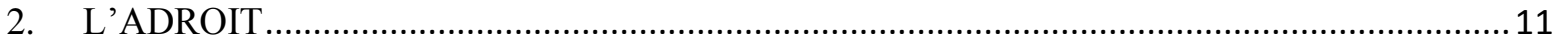

3. Spaceborne, Pulsed UV Laser System .............................................................................. 13

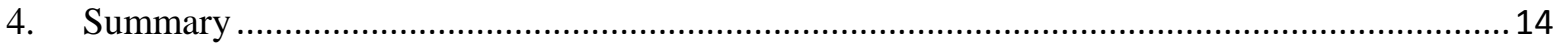

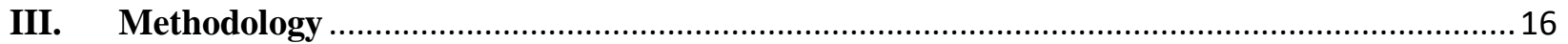

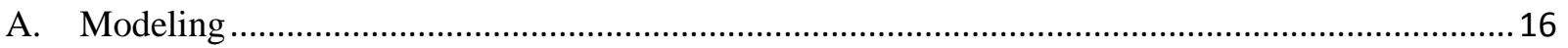

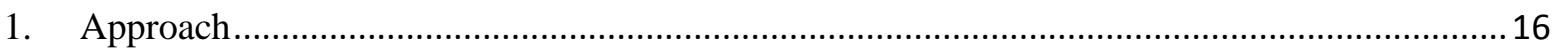

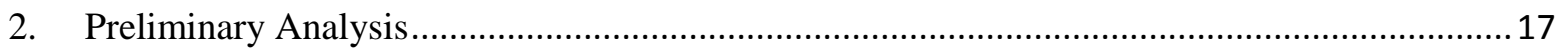

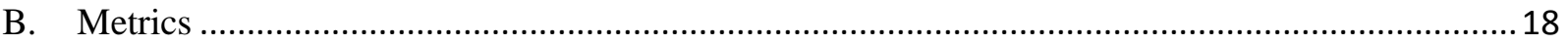

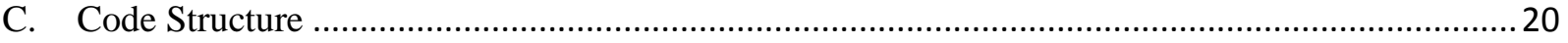

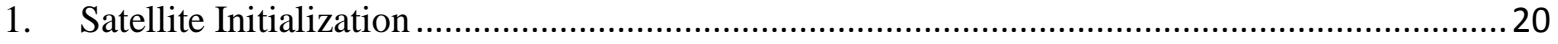

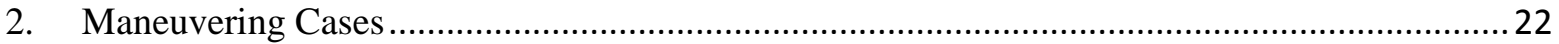

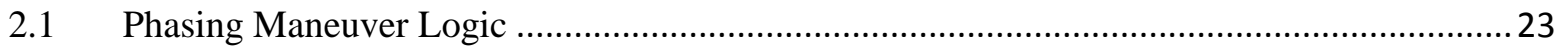

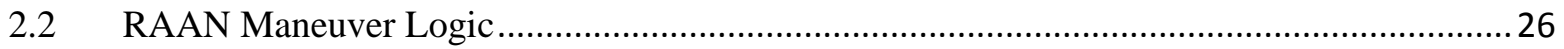

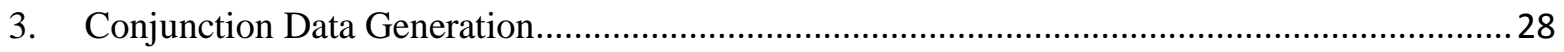

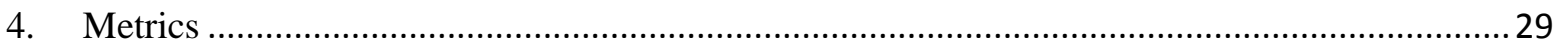

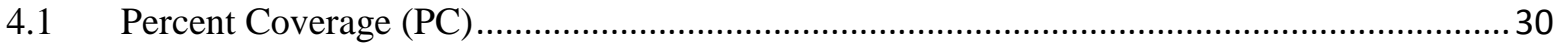

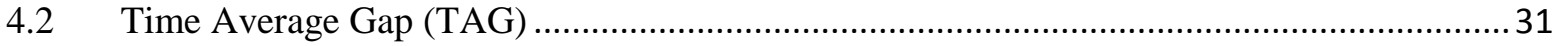

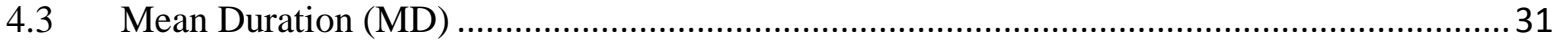

$4.4 \quad$ Mean Response Time (MRT) …................................................................................... 31

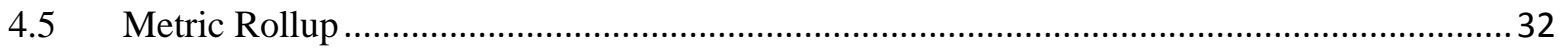

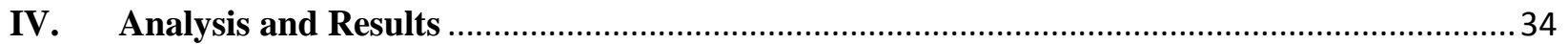

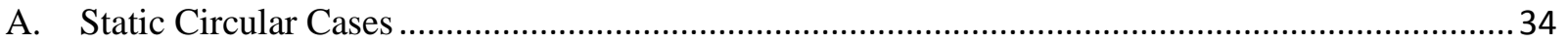

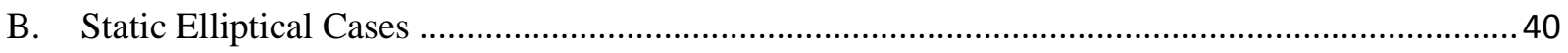




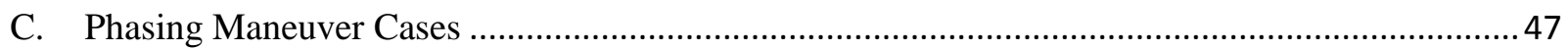

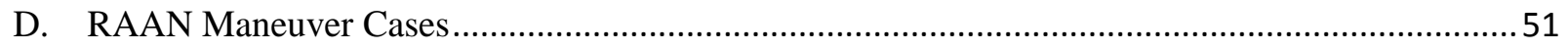

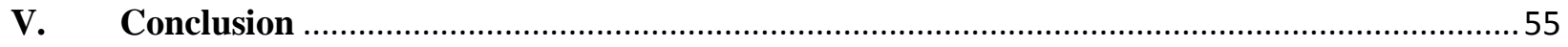

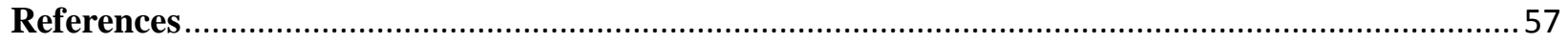

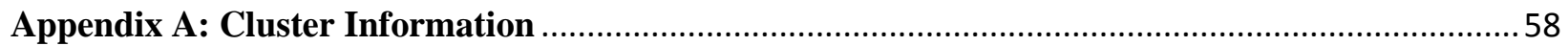

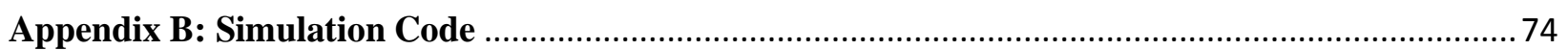




\section{List of Figures}

Fig. 1 Cluster Spatial Density and Location, with Total Cluster Mass [2] ......................................................6

Fig. 2 L'ADROIT LDR System for Orbital Debris Removal. [4] .................................................................11

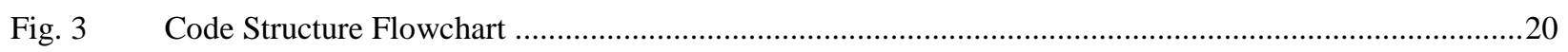

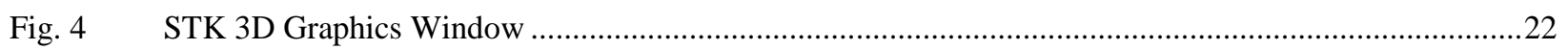

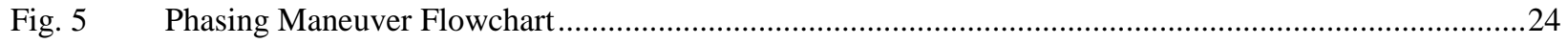

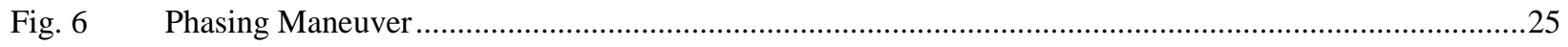

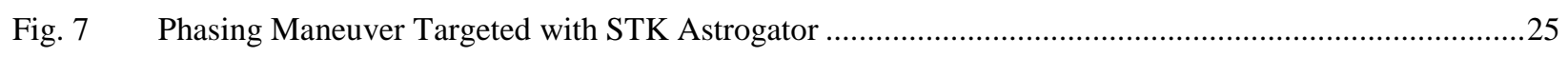

Fig. 8 RAAN Maneuver, Changing the Node [12] ......................................................................................22

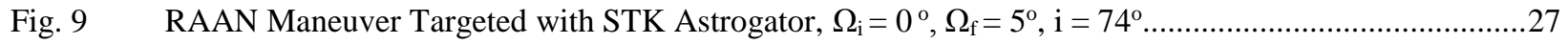

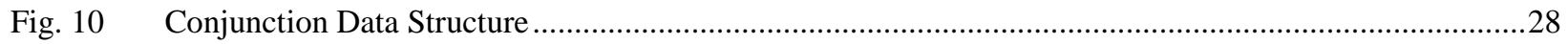

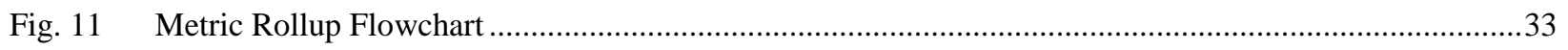

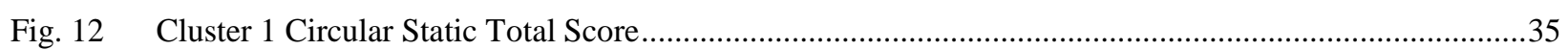

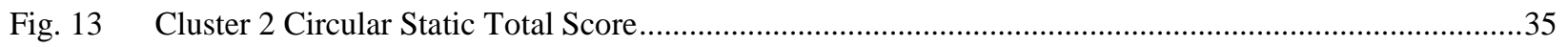

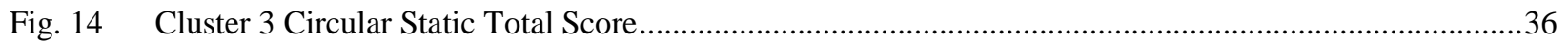

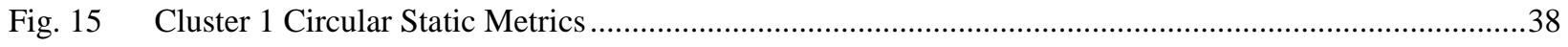

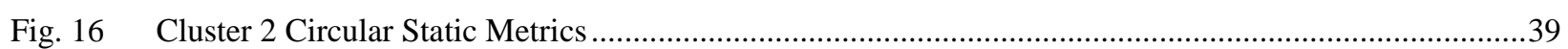

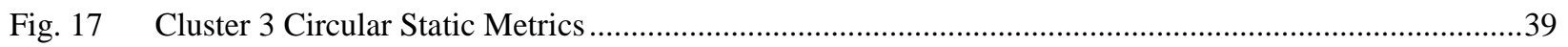

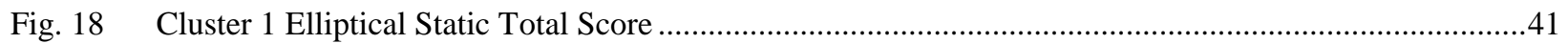

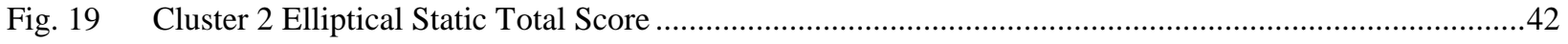

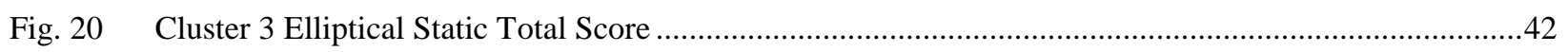

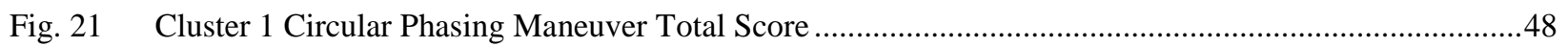

Fig. 22 Cluster 1 Elliptical Phasing Maneuver Total Score ……….................................................................48

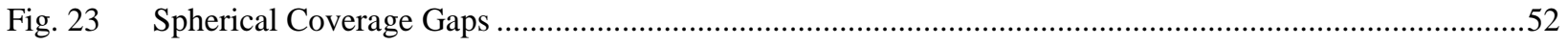

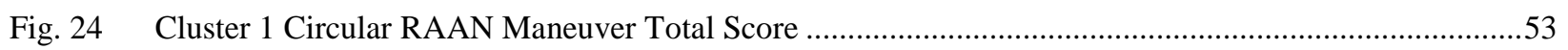

Fig. 25 Cluster 1 Elliptical RAAN Maneuver Total Score …................................................................................53 


\section{List of Tables}

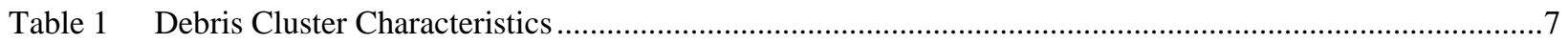

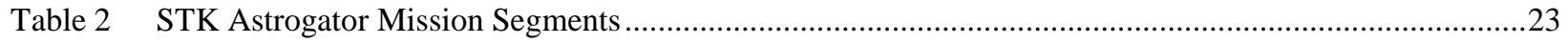

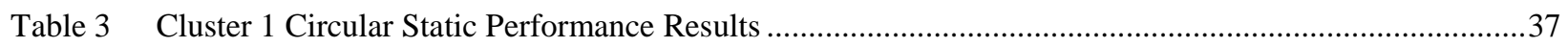

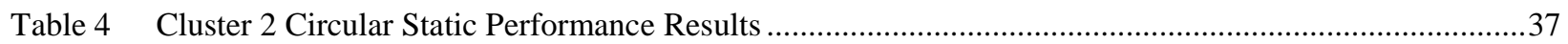

Table 5 Cluster 3 Circular Static Performance Results ......................................................................................38

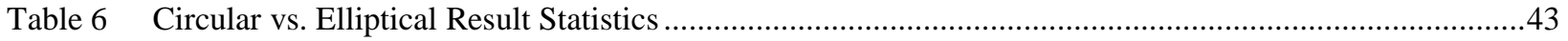

Table 7 Cluster 1 Elliptical Static Performance Results.........................................................................................44

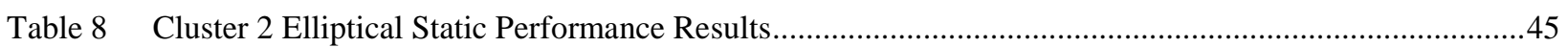

Table 9 Cluster 3 Elliptical Static Performance Results......................................................................................46

Table 10 Cluster 1 Circular Phasing Maneuver Performance Results ...............................................................49

Table 11 Cluster 1 Elliptical Phasing Maneuver Performance Results................................................................50

Table 12 Cluster 1 Circular RAAN Maneuver Performance Results ...................................................................54

Table 13 Cluster 1 Elliptical RAAN Maneuver Performance Results .................................................................54 


\section{Introduction}

\section{A. Background}

It is globally accepted that orbital debris is a growing hazard that threatens space operations and the future of space traffic management. In 2017, The United States Congress acknowledged as part of the NASA Transition Authorization Act, S.442*, that “... orbital debris poses serious risks to the operational space capabilities of the U.S.; an international commitment and integrated strategic plan are needed to mitigate the growth of orbital debris wherever possible." There have already been multiple documented events in which debris impact has resulted in the termination of an active satellite. Numerous other spacecraft anomalies are also suspected to have been caused by collisions with untracked debris.

A collision between space debris objects would have an immediate impact on space flight safety, causing a deterioration of payload operations and a reduction in the operational lifetimes of satellites. Past satellite collisions have already demonstrated the amount of additional debris that is generated from a high velocity impact. The Iridium-Cosmos collision in 2009 involved a total mass of $1600 \mathrm{~kg}$ and produced over 3000 trackable fragments and likely 30,000 non-trackable fragments.[1,2] Further, the 850kg Fengyun-1C destruction resulted in roughly 2,200 trackable fragments and likely over 25,000 non-trackable fragments.[2] The debris from theses collisions will remain in orbit for decades, creating an ongoing collision threat for objects in Low Earth Orbit (LEO).

The amount of debris created from a collision is directly proportional to the total mass involved, meaning increased attention should be placed on the most massive at-risk objects. While much of the orbital debris collision risk is usually assessed by looking at the full volume of LEO, most

\footnotetext{
${ }^{*}$ S.442 - National Aeronautics and Space Administration Transition Authorization Act of 2017. $115^{\text {th }}$ Congress. Public Law No: 115-10 (03/21/2017)
} 
massive debris objects are located in tightly clumped clusters with a higher probability for collision due to their overlapping altitudes and inclinations. These clusters are composed of mostly abandoned rocket bodies that are up to $8,300 \mathrm{~kg}$ each. Three of these clusters account for almost $30 \%$ of all derelict mass in LEO. The massive size of these objects combined with the increased probability of collision makes these clusters one of the most immediate risks posed by orbital debris.[2]

To properly address this looming threat, a new approach to space traffic management must be developed that can monitor and characterize these massive debris objects in their operational environment, while simultaneously focusing on preventing a catastrophic collision that could hinder space operations for decades.

\section{B. Problem Statement}

Ground based monitoring of these debris clusters is not adequate enough to properly mitigate their inherent risk. Space based sensors can improve Space Situational Awareness (SSA) capabilities beyond what is achievable with ground based sensors. Close up characterization can help identify if large derelicts have existing damage from collisions with non-trackable debris, while monitoring for new damage in an effort to delineate the location of currently non-trackable debris. In addition to providing a higher level of monitoring and characterization, a space based SSA constellation integrated within the debris clusters allows for faster intervention in the event a potential collision is determined.

The risk of collision between the massive objects within these clusters can be properly mitigated with a system capable of reliably performing Just-In-Time Collision Avoidance (JCA). This method involves predicting potential collisions and intervening by nudging the orbit of one of the involved objects. Various methods of performing JCA have been explored for a number of years, 
such as deploying talcum powder or a cloud of gas in a satellites path via sounding rockets.[3] One of the more promising and economically viable options for JCA is Laser Debris Removal (LDR) in which a laser is used to heat up material on a derelict satellite, creating an ejection of plasma roughly perpendicular to the surface.[4] This ejection imparts momentum onto the debris, effectively raising or lowering its orbit. LDR can be performed over a wide scope of ranges, and could be effectively applied by a constellation of observer satellites.

One of the challenges with developing SSA capabilities is determining what level of coverage and interaction between the observer satellites (OBS) and the resident space objects (RSO) is adequate. Any constellation of observer satellites must be able to monitor every object within the cluster with enough quality and timeliness to accurately predict all potential collisions, but also provide a full scope LDR system capable of reliably intervening. The principal challenge for LDR systems operating in the LEO environment is the relative velocities between OBSs and RSOs, which make it difficult to maintain access for a long enough duration to fully re-position noncooperative debris.

Research reported in "Geostationary Orbit Development and Evaluation for Space Situational Awareness" [5] developed a methodology for evaluating near-GEO observation satellite constellations to optimize their SSA capabilities. We have adapted and improved this methodology to evaluate different constellation designs for the purpose of determining the optimal configuration capable of mitigating the collision risk within the debris clusters in LEO. Different approaches, including circular, eccentric, and self-maneuvering designs are considered.

This research focuses on analyzing the methods for evaluating the performance of LEO SSA and collision intervention LDR of space debris clusters. While specific focus is placed on three particular debris clusters, the intent is not to fully develop a sensor suite and LDR system. But 
rather, focus on a broad approach to highlight the achievable coverage of an observation constellation, demonstrate the collision mitigation potential, and further develop a methodology that can be applied to any SSA or interaction-based spacecraft constellation. Range, illumination, and line-of-sight based criteria will be used to assess periods of access between the OBS constellation and debris RSOs, derived from researched LDR operating constraints.

\section{Research Objectives, Questions, and Hypothesis}

The primary aim of this research is to zero in on a constellation architecture that can most effectively mitigate the collision risk within the debris clusters by providing timely, quality, and efficient coverage. To achieve this goal, a number of research questions need to be answered. Are there certain classes of constellation designs that outperform in any of the grading metrics? This will provide insight into niche designs that could be effectively employed for specific SSA needs, such as a cluster with highly inclined objects. Additionally, will maneuvering constellations improve performance in any metrics, and will those improvements justify their fuel and $\Delta V$ costs? Maneuvers can be costly, especially when constant maneuvering is expected. Even if maneuvering satellites improve the SSA coverage of a constellation, are the $\Delta V$ requirements too prohibitive to consider feasibly implementing?

We hypothesize that an investigation of constellation design capabilities will result in multiple options that are able to meaningfully impact the threat orbital debris poses to space operations and economic growth. Further, the investigation will demonstrate the range of applications that the tools and methodology showcased in this research have with regards to addressing SSA and space traffic management needs. Our research shows that increasingly complex designs result in improved performance for some grading metrics, but designs that more closely mirror the specific orbits of the objects being observed offer the best results. We expect designs that implement 
maneuvers will improve the overall coverage of the debris clusters, but that the improvements are tempered by the significant fuel costs. Cost and mission lifetime are important factors in a mission's ultimate success. Finally, while the same constellation architecture is expected to provide the best performance for all three clusters analyzed, it is hypothesized that the specific altitude and eccentricity that best meets the mission criteria will be different for each cluster, based on their differences in altitude, inclination, and derelict RSO dispersion. 


\section{Background}

\section{A. Debris Clusters}

For this research effort, the constellation design methodology will be applied to three independent debris clusters. Satellites are considered a 'cluster' when they orbit with small variations in their inclinations and altitudes. Analyses have shown that interaction between the members of these clusters is higher than typical satellite interaction statistics indicate.[4] The close proximity of the objects leads to a higher probability of collision, while the massive size of the objects magnifies the scientific and economic impact a potential collision would have on space operations in their vicinity.

Figure 1 shows the spatial density, total mass, and average altitude of each cluster. The combined mass of the clusters accounts for close to $30 \%$ of the total derelict mass in LEO.[2] Table 1 contains basic characteristics of the clusters being evaluated, including the probability of collision in one year, and the estimated debris produced from a collision. The clusters are comprised of mostly Russian rocket bodies, though other payloads also contribute to the high mass concentration. A detailed list of all objects included in each cluster is provided in Appendix A.

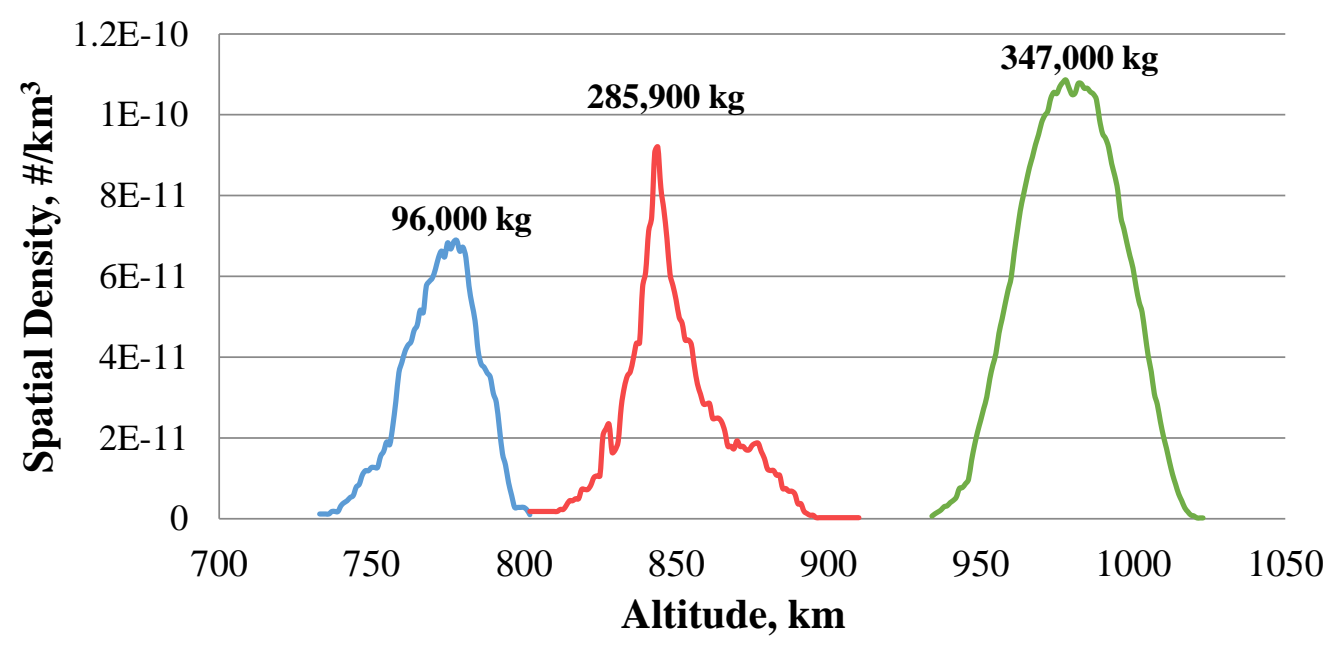

Fig. 1 Cluster Spatial Density and Location, with Total Cluster Mass [2] 
Table 1 Debris Cluster Characteristics

\begin{tabular}{|c|c|c|c|c|c|c|c|c|c|c|c|}
\hline & & & & & \multicolumn{2}{|c|}{ Altitude Extremes } & & \multicolumn{2}{|c|}{ Produced From a Collision $^{3}$} \\
\hline $\begin{array}{l}\text { Cluster } \\
\text { Altitude }\end{array}$ & Cluster & $\begin{array}{l}\text { Approximate } \\
\text { Mass }(\mathrm{kg})^{1}\end{array}$ & $\#$ & $\mathrm{~L} / \mathrm{D}(\mathrm{m})$ & High & Lowest & 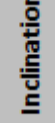 & $\begin{array}{c}\text { Mass Span } \\
(\mathrm{kg} / \mathrm{km})\end{array}$ & PC/Year ${ }^{2}$ & Cataloged & $\begin{array}{c}\text { Lethal, } \\
\text { Nontrackable } \\
\text { (LNT) }\end{array}$ \\
\hline \multirow{2}{*}{$775 \mathrm{~km}$} & SL-8 R/B & 1,434 & 44 & $7 / 2.4$ & 793 & 733 & 74 & 1052 & \multirow{2}{*}{$1 / 413$} & 2868 & 43020 \\
\hline & SL-8 Payloads & 850 & 44 & $3 / 2$ & 802 & 742 & 74 & 623 & & 3400 & 42500 \\
\hline \multirow{3}{*}{$850 \mathrm{~km}$} & SL-16 R/B & 8,300 & 18 & $11 / 3.9$ & 860 & 814 & 71 & 3248 & \multirow{3}{*}{$1 / 548$} & 16600 & 249000 \\
\hline & SL-16 Payloads & 3,250 & 18 & $4 / 2$ & 868 & 823 & 71 & 1300 & & 13000 & 162500 \\
\hline & Other Payloads & 2,000 & 39 & $4 / 2$ & 910 & 795 & 80 & 678 & & 8000 & 100000 \\
\hline \multirow{3}{*}{$975 \mathrm{~km}$} & SL-8 R/B & 1,434 & 144 & $7 / 2.4$ & 1020 & 935 & 83 & 2429 & \multirow{3}{*}{$1 / 92$} & 2868 & 43020 \\
\hline & SL-8 Payloads & 800 & 142 & $3 / 2$ & 1024 & 934 & 83 & 1262 & & 3200 & 40000 \\
\hline & Other Payloads & 1500 & 18 & $4 / 2$ & 997 & 905 & 64 & 293 & & 6000 & 75000 \\
\hline
\end{tabular}

1. Mass and size values are approximate as the variety of sources used did not have identical consensus - there is some uncertainty in these values. Where there are different mass objects, the approximate mass represents an average.

2. Probability of Collision (PC) is calculated using a 'Probability Calculator', based on number of objects, collision cross section, collision impact velocity, exposure time, volume of cluster, etc.[2]

3. Rocket Body (R/B) on R/B collisions use $1 \mathrm{x}$ mass for cataloged object production (but also $2 \mathrm{x}$ to capture both objects involved) and $15 \mathrm{x}$ mass for $\mathrm{LNT}$. Payload (P/L) on P/L uses $2 \mathrm{x} / 25 \mathrm{x}$ for catalogued/LNT and R/B on P/L uses 1.5x/20x. [2]

\section{A single collision between two $8300 \mathrm{~kg} \mathrm{SL-16}$ rocket bodies would produce an estimated 16,600}

cataloged objects, and an estimated 249,000 lethal non-trackable (LNT) objects in its vicinity.

Catalogued debris is any object greater than $10 \mathrm{~cm}$, while LNTs are any debris objects smaller than $10 \mathrm{~cm}$. This collision would have a much larger impact on space operations than any other recent collision event, as it would nearly double the current catalogued debris population, which is approximately 23,000 objects. The $975 \mathrm{~km}$ cluster has the highest probability for collision. With 304 objects, the cumulative probability of collision each year is $\sim 1 \%$. Debris created from a collision in either of these clusters will remain in orbit for decades. This much debris would impact 
all satellites near the collisions orbit, and create a hazard for any space vehicle traveling through the debris field. Due to the similar orbits of the objects within these clusters, a single collision could lead to a runaway effect causing further impact and generating even more debris.[6]

Despite the increased risk and consequence of a collision, no group is specifically monitoring collision potential within these clusters.[2] The Combined Space Operations Center (CSpOC) is tasked with monitoring possible collisions with all operational satellites, but no attention is currently given to collisions between derelict satellites despite only $6 \%$ of catalogued space debris being operational.[6] The potential impact on operational satellites from a collision between massive derelicts makes them just as important to monitor.

\section{B. Laser Debris Removal}

Laser ablation has been studied for the purpose of orbital debris mitigation for over 25 years. Materials, if heated above a specific fluence threshold $\left(\mathrm{J} / \mathrm{m}^{2}\right)$, will vaporize and eject plasma, imparting momentum onto the target.[7] From ground-based to space-based systems, lasers provide a promising and economic means for reducing orbital debris impact. ORION, a NASA ground-based concept validation study concluded that LDR is not only feasible, but is economically modest compared to alternative debris mitigation options, such as shielding or replacing high-value spacecraft.[8, 9] In a 2014 study, the International Coherent Amplifying Network (ICAN) developed a fiber based laser architecture to de-orbit small debris. They concluded that space-based operation of LDR systems is achievable and efficient.[10] Based on their success, ICAN researchers held the first workshop to discuss laser solutions for orbital debris in 2015 .

Space based LDR systems provide many advantages over ground based systems. The space environment provides a comparatively large target access rate, which in turn permits the system to 
use smaller and lighter optics while providing superior performance. Space also provides a black background, making for better target detection and acquisition. The main advantage a space based system offers, though, is in reducing the operating range between laser aperture and target. A shorter range reduces the average operating power, since the laser pulse energy is more efficient at producing momentum on the target.[7]

The measure of laser pulse energy efficiency is captured in the coupling factor $\left(\mathrm{C}_{\mathrm{m}}\right)$, Eq. (1), defined by laser radiation parameters including the irradiation $I\left(\mathrm{~W} / \mathrm{m}^{2}\right)$, pulse duration $\tau(\mathrm{s})$, and wavelength $\lambda(\mathrm{m}) .[7]$

$$
C_{m}=\frac{C_{m 0}}{(I \cdot \lambda \cdot \sqrt{\tau})^{1 / 4}}
$$

$\mathrm{C}_{\mathrm{m} 0}$ is a coefficient dependent on the target material, as a function of the average atomic mass and charge state in the ablated plasma.[7] While radiation parameter selection is crucial in the successful application of LDR systems, adjustments made on orbit mean a single system is capable of adapting to handle different sizes of debris and operating at different ranges. For larger targets, the same laser optics can be effective by increasing the pulse energy needed for smaller targets. Beam focal spot size can also be adjusted to generate the optimal fluence for any specific target.

The coupling factor is a direct measure of how effective the laser optics are at changing the targets velocity. Based on this value, Eq. (2) can be used to calculate $\Delta V$ capabilities of the optics; $\mu\left(\mathrm{kg} / \mathrm{m}^{2}\right)$ represents the targets mass density, $\Phi\left(\mathrm{J} / \mathrm{m}^{2}\right)$ represents fluence, and $\eta_{c}$ represents an impulse transfer efficiency term based on thrust direction and target orientation.[7] 


$$
\Delta V=\frac{\eta_{c} \cdot C_{m} \cdot \Phi}{\mu}
$$

\section{Literature Review}

The intention of this research is not to develop a fully designed LDR system, but rather to contrast different constellation designs to find the architecture that provides the best platform for space based SSA and LDR mitigation within debris clusters. Literature review is relied on to provide reference and context for expected operating ranges, laser engagement duration expectations, and $\Delta V$ capabilities for the high mass derelict objects within the clusters. These reference metrics will help guide the constellation judging criteria, and dictate access constraints such as range and illumination.

Two primary research articles were relied on as references with respect to space based orbital debris mitigation. First, "L'ADROIT - A spaceborne ultraviolet laser system for space debris clearing" [4] by Phipps and Bonnal develops a high fidelity LDR system capable of handling small and large debris. The detail of L'ADROIT (Laser Ablative Debris Removal by Orbital Impulse Transfer) incorporates realistic target detection rate, minimum and maximum operating ranges for

small and large debris, and per-object cost estimates for nudging and removal. Further, " $A$ spaceborne, pulsed UV laser system for re-entering or nudging LEO debris, and re-orbiting GEO debris, '[7] also by Phipps, provides an in-depth look at how the L'ADROIT system can be tweaked and applied to orbital debris mitigation on multiple levels. Various approaches to large derelict nudging are discussed, including LEO collision avoidance and GEO debris re-orbiting. Range and duration requirements for debris nudging on the scale of $10 \mathrm{~km}$ to $300 \mathrm{~km}$ are examined. 


\section{L'ADROIT}

In L'ADROIT, Phipps states that "pulsed laser active debris removal using laser ablation jets on target is the most cost-effective way to re-enter [debris]". LDR removed the need for orbit matching that most other debris removal methods require. Phipps claims that the L'ADROIT system would cost roughly $\$ 280,000$ to alter a 1 -ton object's orbit by $40 \mathrm{~km}$.[4]

L'ADROIT consists of two solar powered sensors; a wide field of view passive sensor for target acquisition and a narrow field of view active laser for tracking and firing. The acquisition sensor has a 60 degree field of view, with a $70 \mu \mathrm{rad} /$ pixel resolution that identifies targets with solar illumination. Once a target is acquired, the active laser will engage while progressively increasing its pulse energy. The laser pointing direction is guided via feedback based on plasma flashes on the target. Target position along the beam can be determined to $\pm 7.5 \mathrm{~mm}$, giving feedback as to when a target has had its orbit altered by the desired amount.[4] Figure 2 shows the components of the L'ADROIT system, and how the two sensors work together. The zoom mirror helps focus the beam focal spot size to optimize target material ablation.

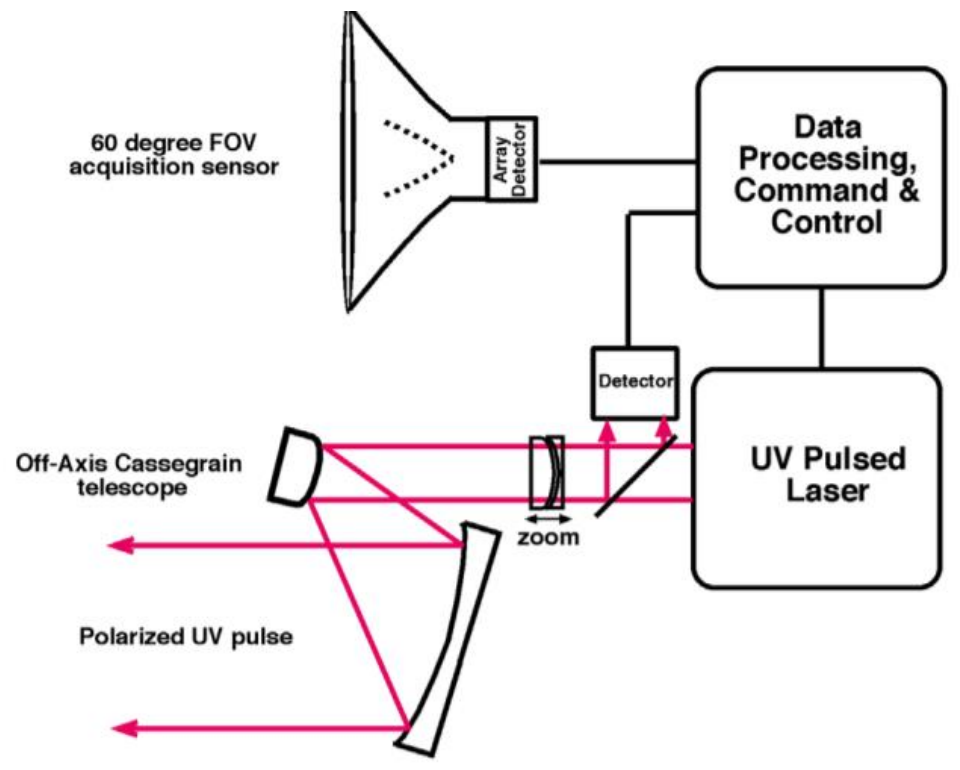

Fig. 2 L'ADROIT LDR System for Orbital Debris Removal. [4] 
A number of factors are considered when determining the optimal operating range for the system. Reasonable pulse energy based on power consumption combined with appropriate laser spot diameter are the key factors. Good acquisition rate based on relative velocities is also important, considering transverse angular rates to maintain line of sight with the target. With all of these factors, the range for large targets is set at $600 \pm 300 \mathrm{~km}$. The lower end of the operating range is chosen primarily based on avoiding transverse angular rates, assuming a relative velocity of $15 \mathrm{~km} / \mathrm{s}$. Though reasonable, this range is chosen based on optics intended for addressing small debris as well. The operating range for small targets is chosen as $250 \pm 75 \mathrm{~km}$. Without the added constraint of optimizing the optics for both small and large debris, the optics could be more finetuned for large debris at longer ranges.[4]

For our research, a minimum range is not used to limit target access intervals, since improving general observation and SSA is also an objective. It is assumed LDR operation will occur during reasonable ranges and windows of operation. While the LDR operating windows may be shorter than recorded object access durations, the primary goal is to contrast the performance of various constellation designs, and longer duration access still implies more robust LDR operation capabilities.

The expected performance for large orbital debris intervention using L'ADROIT is estimated with a typical target $\Delta V$ of $8.3 \mathrm{~cm} / \mathrm{s}$ per 40 s duration of laser interaction with a $1000 \mathrm{~kg}$ target.[4] This means 625 interactions are needed to achieve $43 \mathrm{~m} / \mathrm{s} \Delta V$, which is the target $\Delta V$ needed to reorbit the objects by $40 \mathrm{~km}$. Phipps estimates it would take roughly 4 years to complete this process on 2000 one ton objects. Many of the derelict objects within the evaluated clusters in our research are around $1000 \mathrm{~kg}$ with relative velocities at or below $15 \mathrm{~km} / \mathrm{s}$, making the performance expectations for L'ADROIT an appropriate metric for reference. However, $40 \mathrm{~km}$ is conservative 
when considering the degree of orbit adjust necessary for collision avoidance. Shorter durations and fewer interactions could still be effective in collision mitigation.

\section{Spaceborne, Pulsed UV Laser System}

In this research, Phipps addresses the variety of impacts orbital debris have on space operations. Collisions between massive derelicts threaten space flight safety, small debris are hard to track but still pose a high risk, and large GEO derelicts occupy limited orbital slot availability. The system outlined in L'ADROIT defines an architecture for laser debris removal that can be tuned and adapted to address each unique threat orbital debris poses. Phipps explores specific, conservative radiation parameters for a variety of optics that optimize LDR effectiveness in each area.

Parameter tuning is based around implementation on a space based system, with consideration to typical derelict materials and expected operating ranges. Optimal ranges can be determined based on Eq. (3), defined by the pulse energy $E_{L}(\mathrm{~J})$, optimal fluence $\Phi_{\text {opt }}\left(\mathrm{J} / \mathrm{m}^{2}\right)$, mirror diameter $D(\mathrm{~m})$, wavelength $\lambda(\mathrm{m})$, and non-dimensional beam quality $M^{2}$ of the laser optics. A beam quality of $\mathrm{M}^{2}=1$ is the best achievable, though values closer to 2 are more realistic.[7]

$$
L_{M A X}=\sqrt{\frac{\pi \cdot E_{L}}{\Phi_{o p t}}} \cdot \frac{D}{2 \cdot M^{2} \cdot \lambda}
$$

For the GEO debris removal scenario, it is estimated that large derelicts can be adjusted by 100 $\mathrm{km}$ in roughly 75 days. Within this time frame, however, multiple targets can be engaged, meaning 75 days per derelict re-orbit is a highly conservative time estimate. This includes orbit circularization for any target maneuvered into a graveyard orbit. This scenario considers a $3 \mathrm{~m}$ mirror capable of up to $6.8 \mathrm{~kJ}$ energy pulses, operating at a maximum range of $2400 \mathrm{~km}$.[7] 
For LEO derelict nudging, Phipps considers $10 \mathrm{~km}$ of orbit adjustment sufficient to ensure successful intervention in a potential collision. Engaging the debris from an average distance of $1600 \mathrm{~km}$, nudging a 1000kg object would require 2000 pulses of $3.2 \mathrm{~kJ}$. With the optics described, this would be achievable over 830 seconds of laser engagement. It is worth noting that a single engagement of 830 seconds is not necessary, and that the re-orbiting could be accomplished over multiple passes by the same observer satellite, or multiple engagements from different satellites. For a $1000 \mathrm{~kg}$ object at a $760 \mathrm{~km}$ altitude, the corresponding $\Delta V$ to nudge by $10 \mathrm{~km}$ equals 0.52 $\mathrm{m} / \mathrm{s} .[7]$

\section{Summary}

Some aspects of LDR require further investigation before a fully developed system could be launched. While additional research is necessary though, these obstacles do not threaten the core concept. A key aspect of LDR is how effective the plasma ablation is at imparting momentum onto the target. The plasma ejection needs to be perpendicular to the target, meaning orientation of the vehicle is an important consideration. However, studies have shown that orientation errors and non-orthogonal plasma ejection can be considered as an overall momentum efficiency loss. Even with an efficiency loss, impulses in a counter-velocity direction will still lead to a semi-major axis reduction. Another important factor with object orientation is negating all angular movement to prevent tumbling of the target. This can be accomplished with closed-loop laser tracker control based on observed movement to guide impact zone selection. A common criticism of space laser technology is the difficulty in distinguishing the power beam from weaponry. It is assumed that as long as LDR systems are presented as clearly commercial and are operated in a suitable manner, weapon distinctions will not be a serious concern. Finally, further research is needed into the 
ablation of target materials, such as MLI blankets and thermal protections. Without successful ablation of the target materials, momentum transfer is uncertain.[7]

Implementation of a space based LDR system with an observer satellite constellation inside of high risk debris clusters is feasible, practical, and economical. Though specifics for each cluster are not being determined, such as precise $\Delta V$ requirements, laser duration statistics, and valid operating ranges, the reference metrics stand as appropriate benchmarks from which to extrapolate similar metrics. Three tiers of access ranges are based on the laser engagement estimates discussed in the literature review: $2400 \mathrm{~km}, 600 \mathrm{~km}$, and $100 \mathrm{~km}$. Though $100 \mathrm{~km}$ is less than the low end of the ranges discussed, it still provides a benchmark for close range laser interaction and will better characterize general satellite observation opportunities. Illumination will also be a constraint for target access, only using line-of-sight when the RSO is in direct sunlight or penumbra. Average access duration statistics for each RSO will be evaluated based on total duration time and average duration gap, as a way of ensuring engagements with large time gaps are weighted lower than constellations that provide more consistent coverage. 


\section{Methodology}

\section{A. Modeling}

A combination of Matlab and Systems Tool Kit (STK) is used for the simulation and modeling environment for this research. STK is a software package that allows for complex analysis of satellite systems, and is commercially available and integrates well with other analysis software. In determining the analysis method for this investigation, software tools were chosen that could effectively and accurately model the space environment and output all necessary data. STK is also useful in loading the debris object ephemerides by using TLE sets for any defined simulation timeframe. The TLE sets allow a constellation to be easily evaluated over multiple scenario durations and at any point in the clusters life. Lastly, STK can be commanded and operated via Matlab scripts, which allows for automation of the analysis process and easier management of the resulting data. All data processing and data management was conducted using Matlab and Microsoft Excel.

\section{Approach}

The three-tiered approach to the range constraint for this analysis was chosen to align with the methodology adapted from Ref. 5. Comparing the constellation designs based on their overall performance across all three access ranges ensures the robustness of the constellation is appropriately captured. Each subsequent Tier represents a benchmark for ensuring more efficient LDR capabilities and increasingly exquisite target characterization. The Tiers are easily configurable within the simulation, and can be altered to assess their impact on the success of a constellation design. 


\section{Preliminary Analysis}

When first creating the simulation environment, a number of design options were considered. The first step involved creating randomly generated clusters to refine the metrics and data collection aspect of the simulation. This work was beneficial in achieving a better understanding of the orbital motion of objects within clusters of similar altitudes and inclinations. This preliminary work was also useful for developing the maneuvers used in the constellation designs by exploring what maneuvers would be possible to implement, and which could be most effective at improving constellation performance.

The preliminary analysis stage also involved taking one of the clusters, and varying a number of core simulation parameters to assess their impact on the accuracy of the results. First, the number of observer satellites in the constellation was varied to assess the impact on cluster coverage. The typical constellation evaluated in this research has eight evenly spaced satellites, but every option down to four OBS constellations were considered and compared. The number of satellites in the constellation ultimately had no effect on the relative performance between different cases, but as expected adding observer satellites to the constellation enhances performance across the board. While the number of satellites will have an impact on the performance metrics, it will not impact how cases compare to each other. An assessment of the impact of simulation length was also conducted, comparing constellation performance when run for three, six, and 12 months. Longer scenario timeframes increase the simulation runtime drastically, so reducing the scenario time while maintaining accuracy is important. Results were consistent for all scenario lengths analyzed, indicating constellation performance will not change when examined over a three month time frame versus a 12 month time frame. Finally, the impact of Tier selection was tested to ensure different ranges wouldn't produce wildly different results. Though based on specific ranges 
derived from the literature review, the Tiers are by no means absolute ranges at which each constellation can be considered effective or not, so ensuring constellations would perform relatively similar with alternate Tiers is important.

\section{B. Metrics}

A core aspect of this research is how well the grading metrics capture the performance of the constellations. The metrics characterize the scope of the analysis, so it's important each metric captures a key part of the mission goal and that the relative weighing of the metrics is successfully comprehensive. The intention of this research is to determine the constellation architecture that is best suited for space based SSA and LDR collision mitigation of massive debris clusters. In this sense, the most important design choice is the range to target that defines each Tier. The closer an optical sensor is to a target, the better the characterization is regardless of sensor selection. The same is true for LDR in that regardless of laser parameters, the closer the system is to a target the more efficient the transfer of momentum will be. The range selection for each Tier combined with the selection of metrics must encompass the scope of the mission. A strong performance across all metrics is required for a successful constellation architecture. The four metrics, equally weighed, are Percent Coverage (PC), Time Average Gap (TAG), Mean Response Time (MRT), and Mean Duration (MD).

Percent Coverage is a measure of the overall time-based coverage between the constellation and the debris cluster. The total access durations for each RSO are summed and divided by the total simulation time. Maintaining contact between the RSOs and OBSs is a key aspect of SSA and LDR. A lower percent coverage metric indicates a constellation will have less constant coverage of the debris clusters and overall poorer characterization. 
Time Average Gap is a measure of the mean gap duration averaged over time for each RSO. It is "the average length of the gap we would find if we randomly sampled the system".[11] While percent coverage provides a measure of the total engagement time, TAG makes sure there is consistent coverage throughout the simulation. A constellation with large gaps in RSO coverage is not robust enough to fully mitigate the risk of debris collision. If a predicted collision were to occur during a gap in coverage for both RSOs, JCA would not be possible without maneuvering at least one of the observer satellites.

Mean Response Time "takes into account both coverage and gap statistics in trying to determine the whole systems responsiveness"[11]. MRT is a measure of how effectively a constellation is able to respond to instantaneous requests for LDR or SSA engagement.

Mean Duration is the average of every access interval for each RSO. This metric is particularly important for LDR due to the lengthy total engagement times needed to nudge large derelicts successfully into new orbits. Although PC already acts as a way of comparing the total duration times, longer individual durations means fewer conjunctions are needed to nudge a debris object which leads to overall shorter total engagement time.

These metrics can be updated and shaped to fit the goals of any constellation design analysis. For example, if exquisite characterization of RSOs is a primary mission goal, minimizing the range at closest approach could be prioritized by adding a metric that averages the closest conjunction distances for each RSO. By fitting the metrics for the mission, the methodology used in this research could be applied to any satellite grouping that would benefit from space based coverage to ensure the optimal design is found. Equations defining these metrics are found in section IV.C.4. 


\section{Code Structure}

The primary code is a Matlab script that uses the actxserver function to connect to STK, build the scenario, run the full simulation, and calculate all grading metrics. Data is extracted from STK during the simulation and stored in a data structure with metrics for each RSO in the scenario. The script is set up to easily change the structure of the scenario based on user inputs, including scenario length, cluster selection, maneuver flags, and constellation parameters. Once the scenario is loaded, each RSO records every conjunction with an observer satellite that meets the range, illumination, and line-of-site access criteria over the full scenario time frame. Figure 3 below outlines the flow of information for the simulation process. Each component of the analysis is explained in further detail in the following sections.

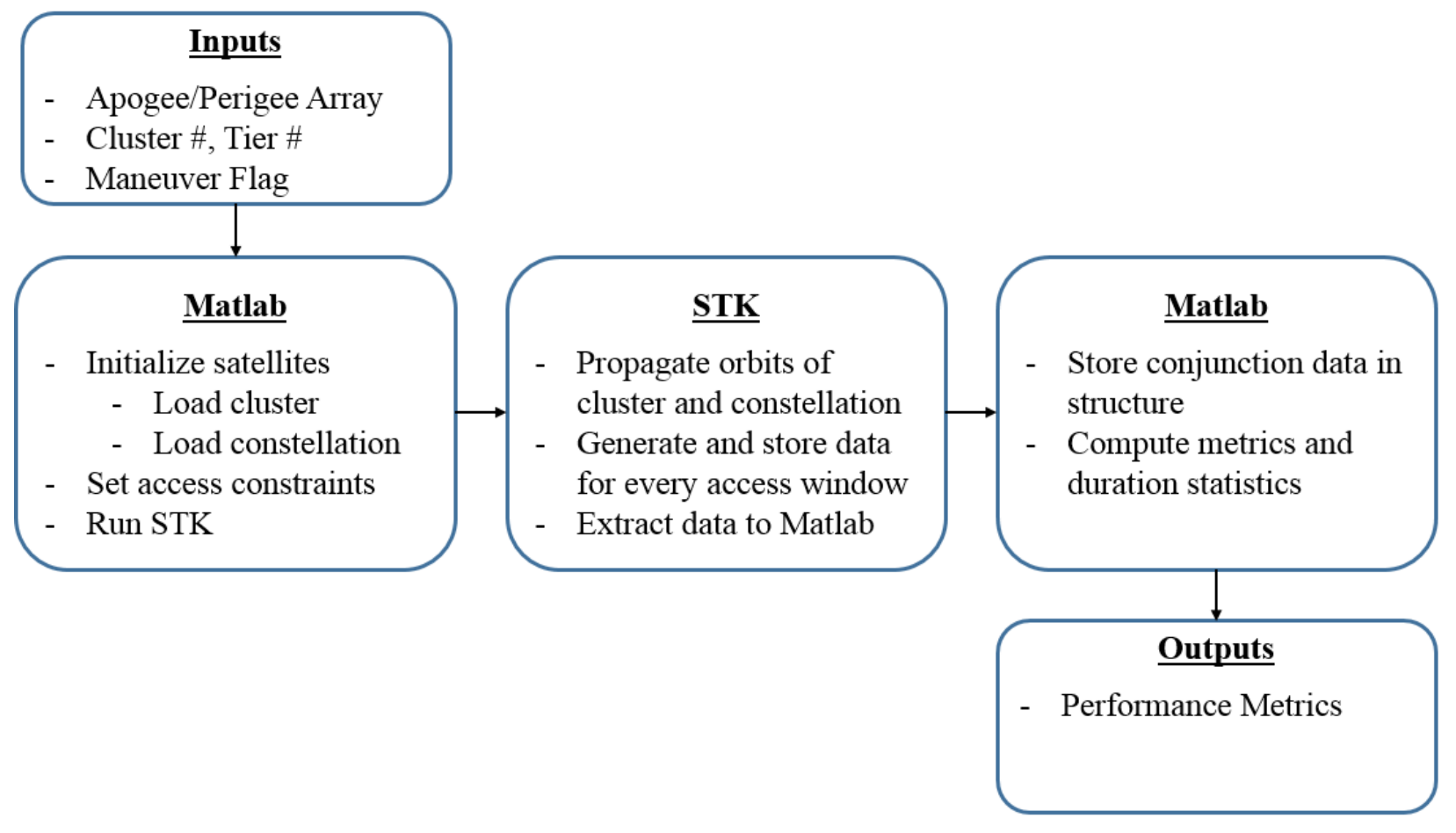

Fig. 3 Code Structure Flowchart

\section{Satellite Initialization}

The first section of the code initializes the selected debris cluster by loading an array of fivedigit satellite catalog numbers into STK. The Simplified General Perturbations (SGP4) propagator 
in STK uses two-line element (TLE) sets to propagate each debris object by generating ephemerides based on the epoch of each TLE. SGP4 considers perturbations due to Earth oblateness, solar and lunar gravitational effects, gravitational resonance effects, and drag induced orbital decay. After initializing in STK, each RSO is configured with range and illumination constraints to define conditions for access intervals with OBSs. The range constraint is set with a maximum distance dependent on the Tier. The illumination constraint is set to only consider a conjunction if the RSO is in either direct sunlight or penumbra, as lighting is a crucial aspect of both LDR engagement and optics based observation.

The observer satellites are then initialized in the simulation based on if they are maneuvering or non-maneuvering. Maneuvering satellites build their trajectories using the STK Astrogator module and will be discussed in more detail in the following section, while non-maneuvering satellites are set up by defining their classical orbital elements (COE) and using the high precision orbit propagator (HPOP). HPOP is a numerical integration propagator that is derived using full algorithms, correct ephemerides, and is the highest fidelity propagator included in STK. Each OBS orbit is defined by an apogee, perigee, inclination, and true anomaly. All satellites in the constellation are evenly spaced around the Earth to most effectively cover the debris cluster. A range constraint is also set to ensure RSO access is limited based on the Tier. Illumination constraints are not necessary since the only lighting requirement is that the debris itself is illuminated from the perspective of the OBS. Figure 4 shows the STK 3D graphics window with Cluster 3 and an 8-object circular constellation loaded and propagated. The 304 debris objects in the cluster have their orbits shown in white, while the OBS constellation has the orbits of its 8 satellites shown in color. The constellation has an inclination of 83 degrees and a central altitude of $7346 \mathrm{~km}$. 


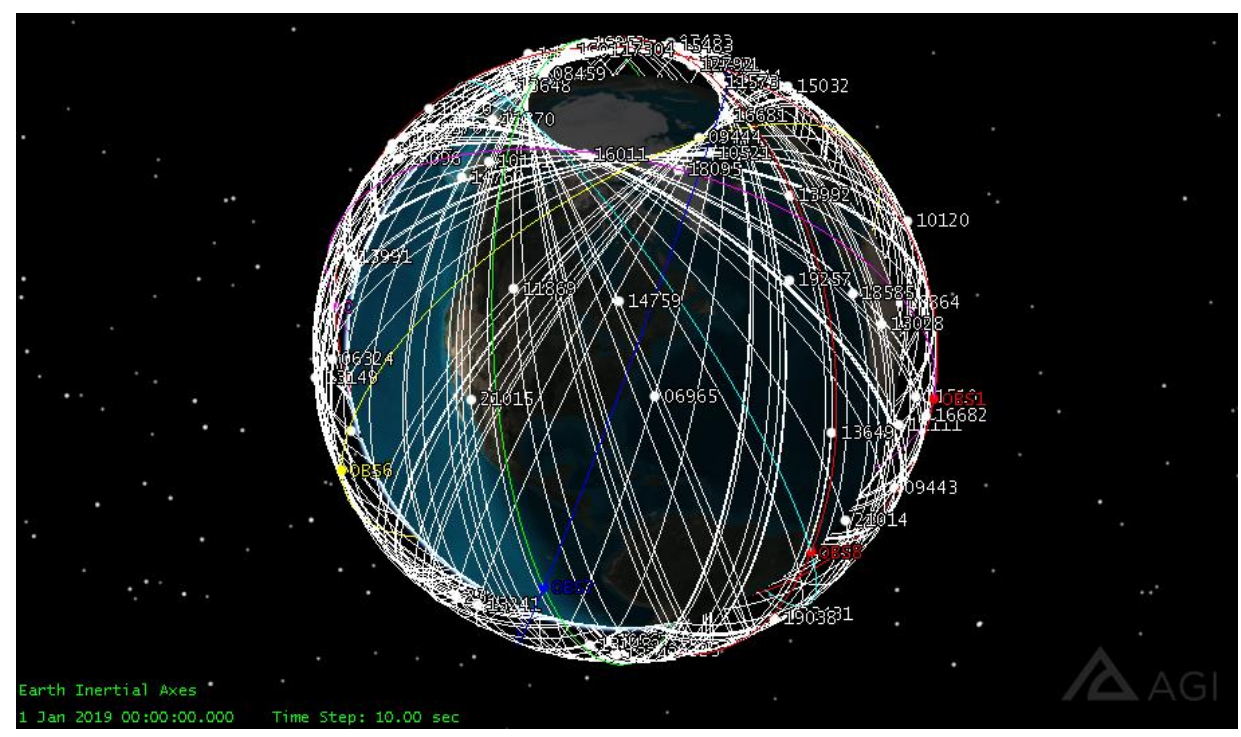

Fig. 4 STK 3D Graphics Window

\section{Maneuvering Cases}

Maneuvering satellites use the STK Astrogator tool, which aids with interactive orbit maneuvering and trajectory design. Astrogator supports a wide variety of options for modeling and targeting satellite trajectories, including impulsive and finite burns, and utilizes HPOP for propagation around the trajectory design. The maneuvering satellites are initialized with COE, propagated to configured stopping conditions, determine the required $\Delta V$ maneuver, and then repeat the process for the duration of the scenario. Maneuvering satellites attempt to improve their engagement with the debris clusters by adjusting their orbits to better interact with the debris. To be effective, however, the performance improvements must justify the $\Delta V$ and fuel usage. Propellant is often a substantial portion of the mass budget for a satellite, and reducing $\Delta V$ requirements extends the life of the mission.

Two types of maneuvers are considered in this research, an in-plane perigee-shift phasing maneuver to adjust the satellites position within their orbital plane and an out-of-plane Right Ascension of the Ascending Node (RAAN) maneuver to rotate the orbital plane. Table 2 identifies 
all of the mission segments for STK's Astrogator tool. These segments are used to create a mission profile for any satellite, and are capable of coordinating conditional maneuvers with a targeting sequence. All setup and commanding for maneuvering cases is handled via the Matlab interface.

Table 2 STK Astrogator Mission Segments

\begin{tabular}{|c|c|c|c|}
\hline Mission Segments & Icon & Definition & Options \\
\hline Initial State & & $\begin{array}{l}\text { Define the initial conditions of the } \\
\text { mission sequence. }\end{array}$ & $\begin{array}{l}\text { Coordinate Frame, Initial } \\
\text { Conditions, Orbit Epoch, Fuel } \\
\text { Tank Parameters, Perturbations }\end{array}$ \\
\hline Launch & $\mathscr{B}$ & $\begin{array}{l}\text { Model a simple spacecraft launch } \\
\text { from Earth or another central body. }\end{array}$ & $\begin{array}{l}\text { Central Body, Ascent Type, } \\
\text { Launch location, Fuel Tank } \\
\text { Parameters, Perturbations }\end{array}$ \\
\hline Follow & & $\begin{array}{l}\text { Set the spacecraft to follow another } \\
\text { vehicle at a specified offset. }\end{array}$ & $\begin{array}{l}\text { Offset, Join and Separation } \\
\text { Parameters, Fuel Tank } \\
\text { Parameters, Perturbations }\end{array}$ \\
\hline Maneuver & & Model a spacecraft maneuver. & $\begin{array}{l}\text { Attitude Control and Orientation, } \\
\Delta V \text { Magnitude, Impulsive vs. } \\
\text { Finite, Engine Type, Fuel Usage }\end{array}$ \\
\hline Propagate & $\theta$ & $\begin{array}{l}\text { Model the movement of the } \\
\text { spacecraft along its current trajectory } \\
\text { until meeting specified stopping } \\
\text { conditions. }\end{array}$ & $\begin{array}{l}\text { Propagator (HPOP default), } \\
\text { Stopping Conditions, Tolerance, } \\
\text { Duration }\end{array}$ \\
\hline Target Sequence & 9 & $\begin{array}{l}\text { Run targeting profiles to converge on } \\
\text { specified satellite conditions. Useful } \\
\text { when stopping conditions are not } \\
\text { easily defined. }\end{array}$ & $\begin{array}{l}\text { Convergence Parameters, } \\
\text { Iterations, Tolerances, Targeting } \\
\text { Parameters }\end{array}$ \\
\hline
\end{tabular}

\subsection{Phasing Maneuver Logic}

The intention of the phasing maneuver is to reposition each satellite in the optimal position within its orbit. Phasing maneuvers are typically used in an attempt to rendezvous with another spacecraft, but are utilized in this research to improve overall performance metrics by repositioning the spacecraft to more reliably conjunct with the debris objects. The observer satellites executing the phasing maneuver will phase their orbit by 10 degrees, orbit for 10 days, and then conduct an analysis to conclude if performance has improved. On the condition performance has improved, it will orbit for another 10 days and then repeat the analysis process. On the condition that 
performance has worsened, it will first perform another 10 degree phasing maneuver and then repeat the process. Over a yearlong simulation, this gives each observer satellite the chance to phase over a full 360 degrees and determine the optimal position within its orbit. Performance over each 10 day period is compared to a running average of the performance up until that point. Any satellite that executes a phasing maneuver is doing so because its performance over the 10 day propagation period has lowered its overall performance metrics. Figure 5 shows an algorithmic representation of the phasing maneuver in a flowchart.

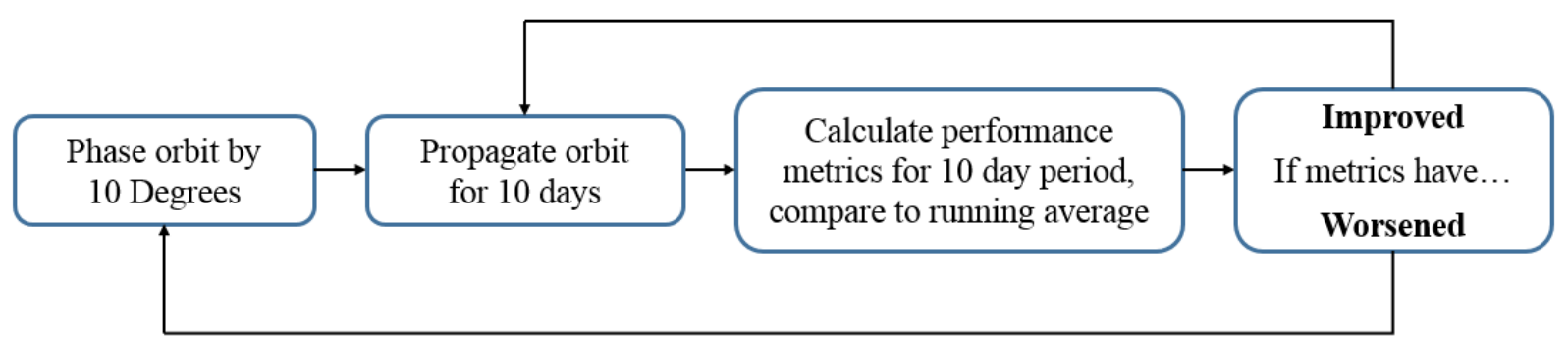

Fig. 5 Phasing Maneuver Flowchart

The phasing maneuver is executed using STK Astrogator's targeting sequence function. Each maneuver involves two burns at apogee that shift the satellites perigee, one to place the satellite in a phasing orbit for a single period and a second to return the satellite to its original orbit. The semimajor axis (SMA) of the phasing orbit is determined by the desired change in period $(T)$. Astrogator's targeting sequence is able to target a desired SMA, and converge on the required $\Delta V$. For the second burn, the original SMA is targeted. Eqs. (4-5) are used to calculate the SMA of the phasing orbit, which is used to target the maneuver.

$$
\begin{gathered}
T_{\text {phasing }}=T_{\text {original }} \cdot\left(\frac{360-\phi}{360}\right) \\
S M A_{\text {phasing }}=\sqrt[3]{\mu_{E} \cdot \frac{T_{\text {phasing }}^{2}}{2 \pi}}
\end{gathered}
$$


In Eq. (4), $\phi$ equals the phasing angle and $\mathrm{T}_{\text {original }}$ is solved for using the original orbits SMA. Figure 6 illustrates a typical phasing maneuver intended to reposition the satellite further along its orbit. Figure 7 shows an example of the maneuver being targeted with STK.

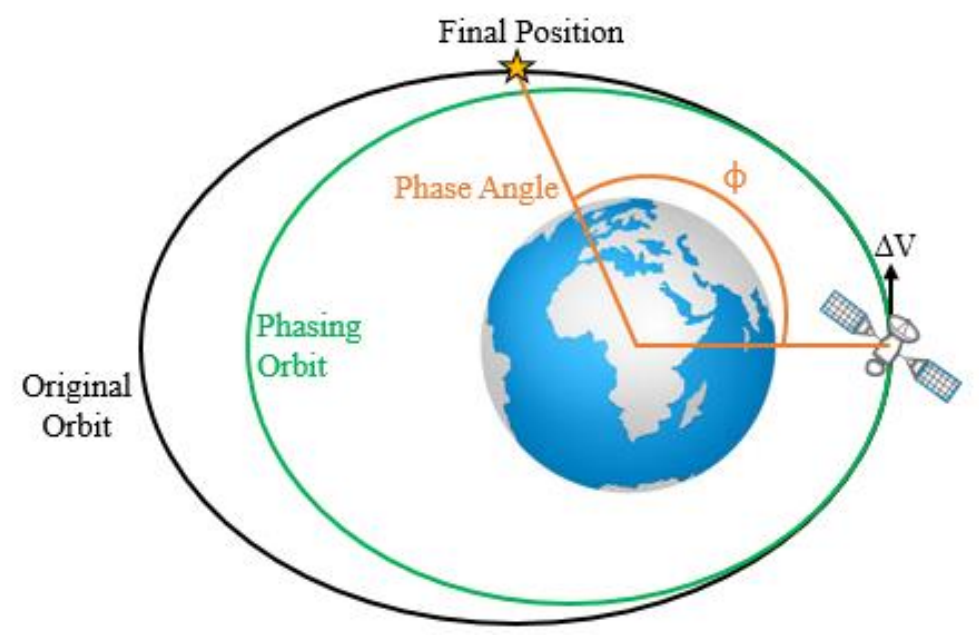

Fig. 6 Phasing Maneuver

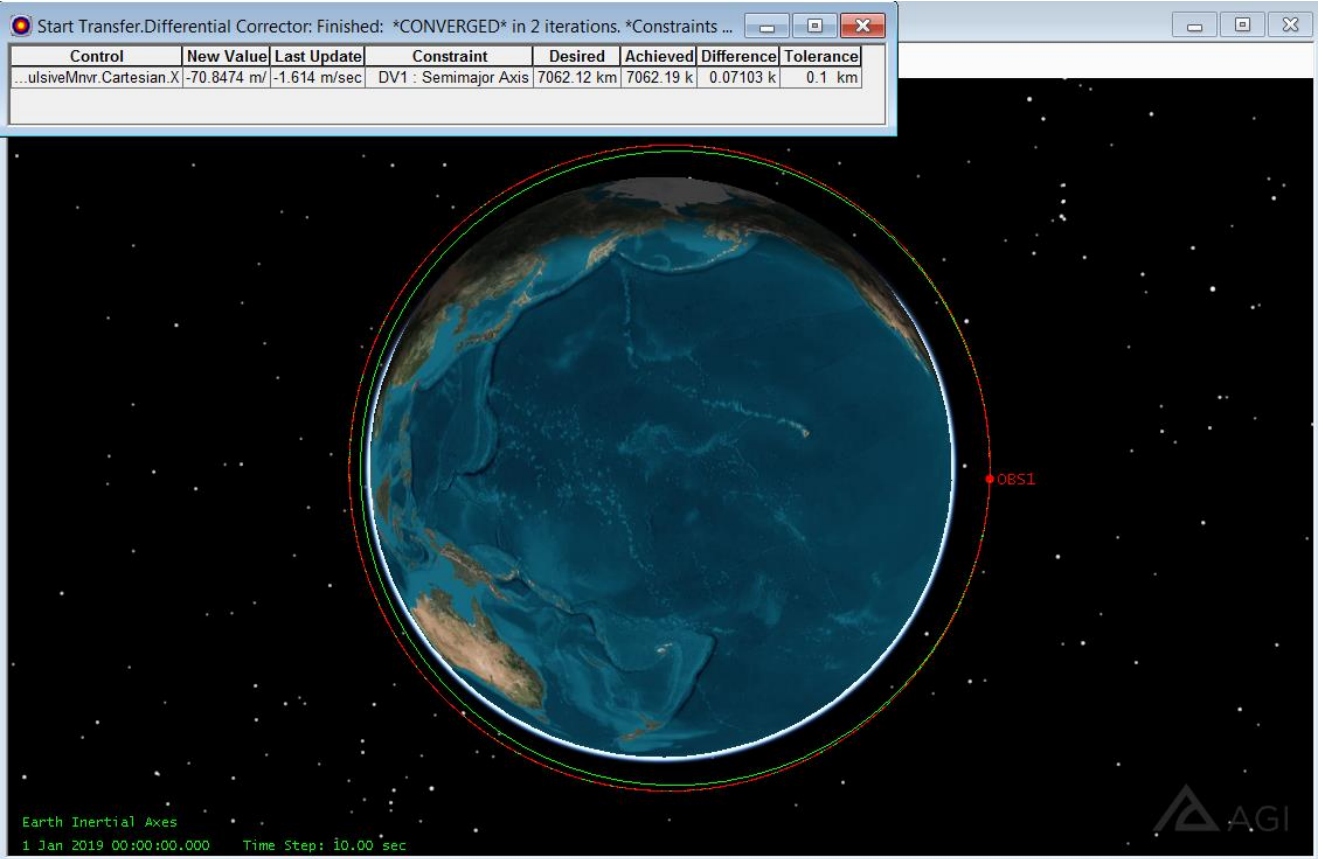

Fig. 7 Phasing Maneuver Targeted with STK Astrogator 


\subsection{RAAN Maneuver Logic}

The intention of the RAAN $(\Omega)$ maneuver is to achieve more comprehensive spherical coverage of the debris clusters. The debris objects in each cluster are spread over a large range of RAANs, requiring out of plane maneuvers to ensure complete coverage. With observer satellites evenly spaced by their RAAN, there are large areas of each cluster that are unmonitored. By continually increasing the RAAN of each satellite by a small amount, the cluster should be able to provide full coverage of the debris clusters. Plane change maneuvers are one of the more costly maneuvers for spacecraft to perform, but necessary to improve spherical coverage of the debris clusters.

Plane change maneuvers can only be executed at the two points of intersection between the original plane and the desired plane. Applying a change in velocity at any point other than the common points will change both the inclination and the RAAN. First, an observer satellite is propagated to the initial argument of latitude $\left(\mu_{i}\right)$ as determined by Eqs. (6-8) [12], based on the inclination $(i)$ and desired RAAN change angle $(\Delta \Omega)$. The Astrogator tool is then used to target the new argument of latitude $\left(\mu_{f}\right)$, with the result converging on the appropriate $\Delta V$ in the out-of-plane direction. For circular orbits, this singular burn results in a pure RAAN maneuver. For elliptical orbits, this maneuver additionally alters the perigee and requires a second burn to then correct back to the initial constellation shape.

$$
\begin{gathered}
\cos (v)=\cos ^{2}(i)+\sin ^{2}(i) \cos (\Delta \Omega) \\
\cos \left(\mu_{i}\right)=\tan (i)\left(\frac{\cos (\Delta \Omega)-\cos (v)}{\sin (v)}\right) \\
\cos \left(\mu_{f}\right)=\cos (i) \sin (i)\left(\frac{1-\cos (\Delta \Omega)}{\sin (v)}\right)
\end{gathered}
$$

In Eqs. (6-8), $v$ represents the angle through which the orbital plane must rotate, and $\mu$ represents the argument of latitude at the point of intersection before and after the maneuver. Eq. (6) is first 
used to solve for $v$, and then Eqs. (7-8) are used to solve for $\mu_{\mathrm{i}}$ and $\mu_{\mathrm{f}}$. Figure 8 illustrates a RAAN change maneuver, and Figure 9 shows the RAAN maneuver being targeted with STK.

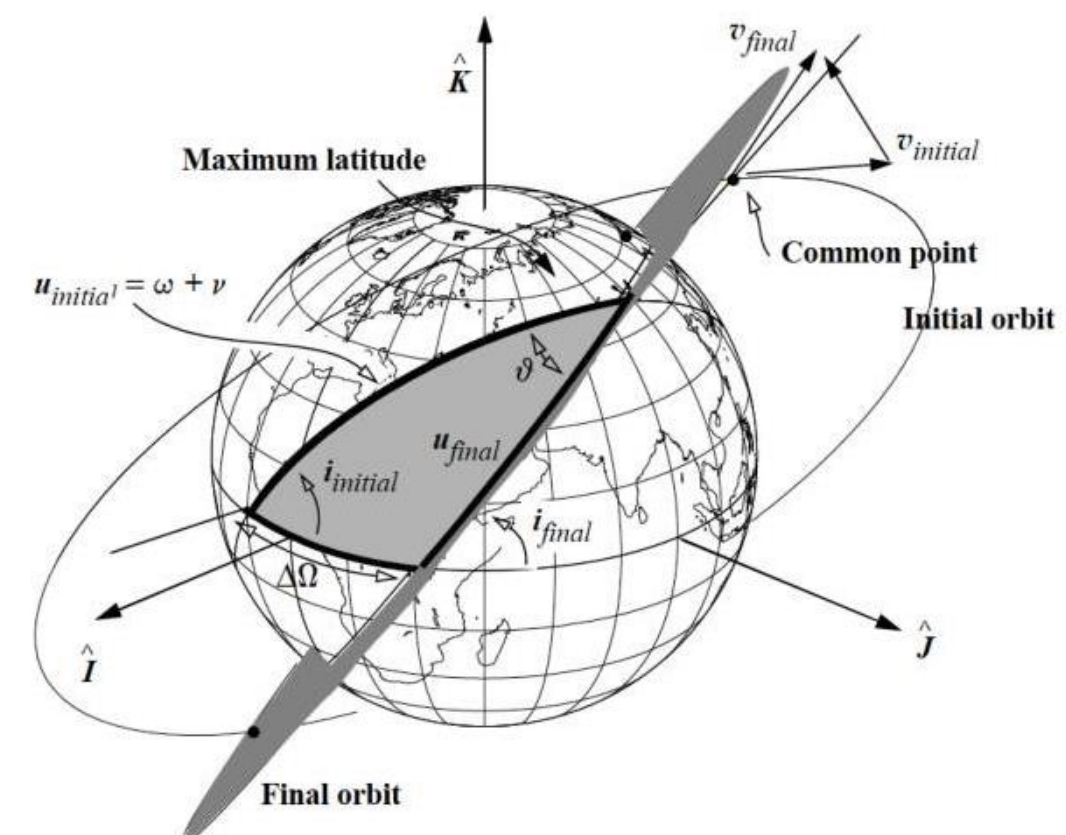

Fig. 8 RAAN Maneuver, Changing the Node [12]

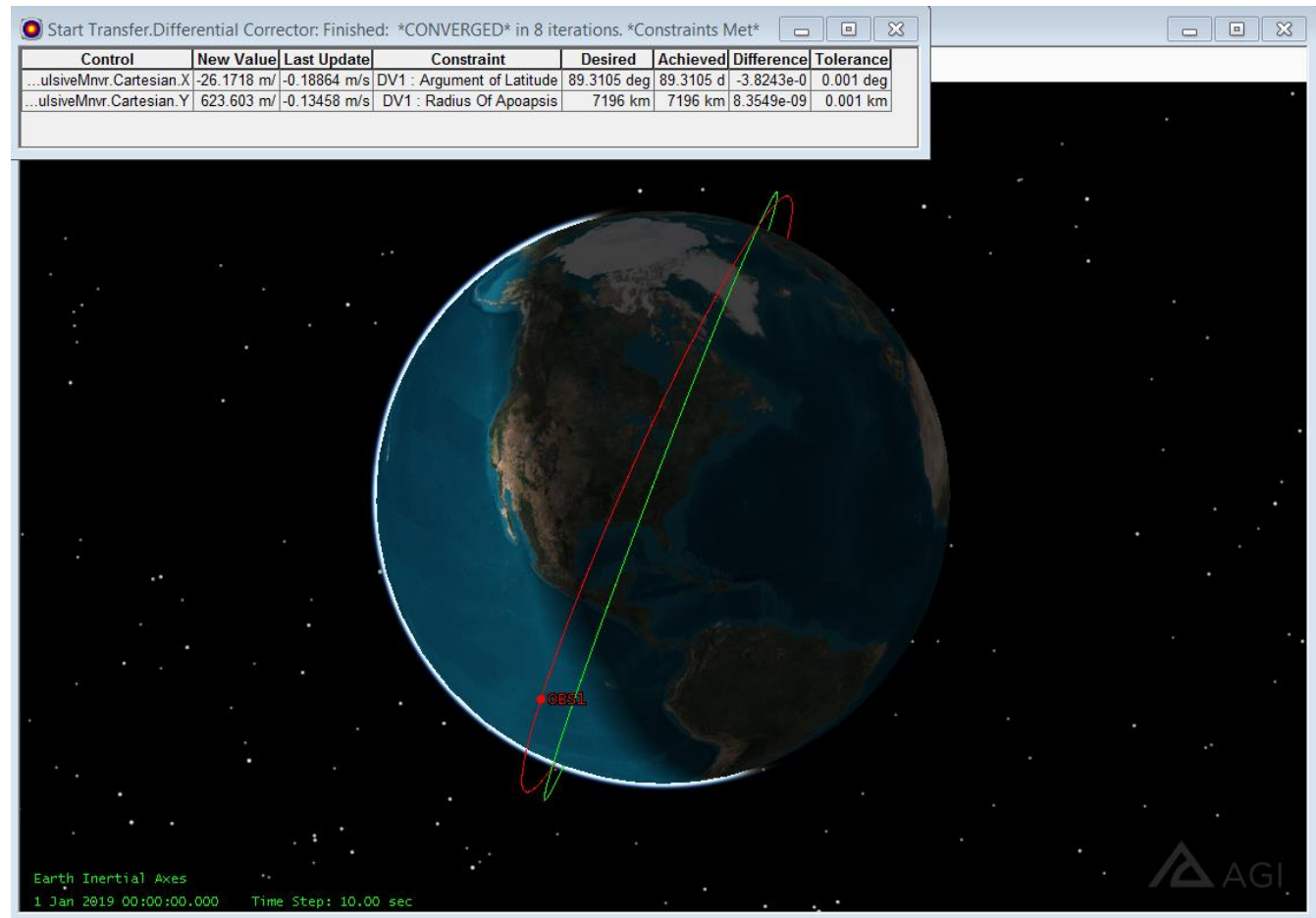

Fig. 9 RAAN Maneuver Targeted with STK Astrogator, $\Omega_{i}=0^{\circ}, \Omega_{f}=5^{\circ}, i=7^{\circ}$ 


\section{Conjunction Data Generation}

The Conjunction Data Generation section loops through each RSO in the debris cluster and computes its access to every OBS in the constellation. This is achieved by creating a displacement vector between the two satellites in the Radial, In-Track, Cross-Track (RIC) frame and corresponding vector magnitude calculation to determine the times and ranges of each access interval. A velocity vector is then created by taking the derivative of the position vector in the Earth-Centered-Earth-Fixed (ECEF) and RIC frames. A wide range of data is collected for each access interval, including total duration, time of closest contact, position, and velocity broken into RIC frame components. The longitude, latitude, and altitude of the RSO at the time of closest approach is also recorded. Figure 10 outlines the data structure used in the analysis.

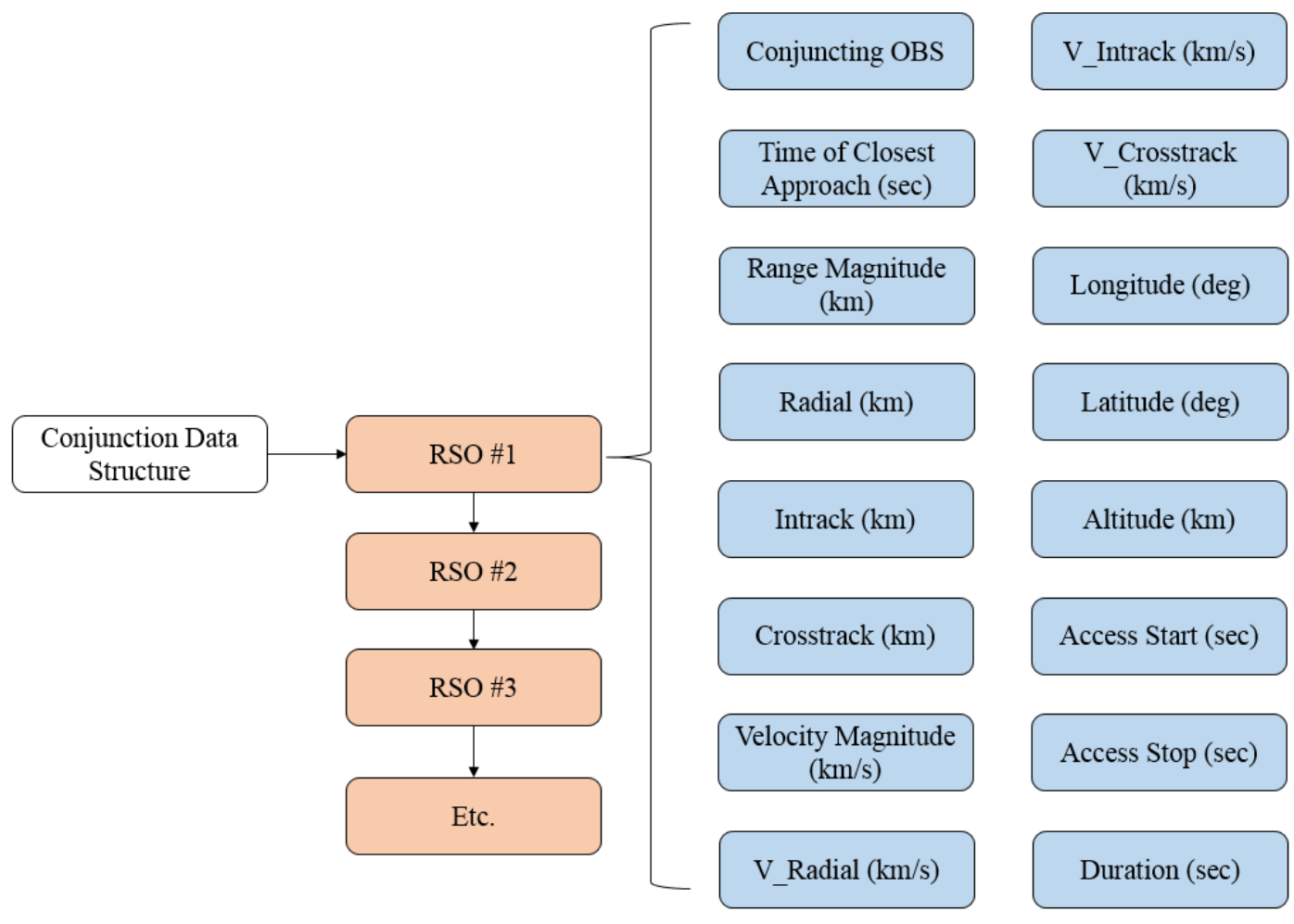

Fig. 10 Conjunction Data Structure 


\section{Metrics}

Once all of the conjunction data has been exported from STK into Matlab, access intervals are sorted in order of occurrence to make it easier to calculate accurate metric statistics. It is common for multiple observer satellites to be in conjunction with the same RSO at the same time, leading to double or triple counting portions of access durations in the percent coverage metric. As conjunctions overlap with each other, they are combined and counted as a single duration for that RSO. While multiple OBSs could theoretically engage an RSO at the same time, it is unlikely that would be part of normal operating procedure. Multiple LDR systems engaging a target at the same time would make it difficult to coordinate beam placement on the RSO so as to avoid canceling out each other's momentum or tumbling the target. In the context of SSA, multiple angles of observation lead to improved RSO characterization, so overlapping conjunctions are indicative of good constellation performance and are not discounted in the other metrics. Mean duration in particular would be skewed if overlapping conjunctions were merged into a single, long access interval. If the final conjunction ends before the end of the simulation, the remaining simulation time is considered a single duration gap. In this way, an RSO with no conjunctions is considered to have a single, long duration gap that equals the full simulation time.

Each constellation is evaluated based on its performance as a whole, and not according to the performance of individual observation satellites. Metrics are first calculated from the perspective of each RSO, and are then aggregated into overall system statistics that represent the performance of the constellation. Statistical analysis of each constellations performance in each metric are also recorded.

Additional metrics for judging the performance of each constellation are calculated, but not included in the official weighing scheme. Metrics that do not contribute to the relative grading 
scheme, but are still important to meet a minimum level of performance. It is crucial that every RSO has some form of consistent access with at least one of the observer satellites. If a constellation does not provide coverage for every RSO in the cluster, it would not meet the requirements of a successful design, even if it were to outweigh other designs in some of the metrics. A cluster conjunction (CC) percentage is calculated and included with the results to capture this behavior. It is also important to remove constellation designs that may be constrained by onboard sensors ability to track an RSO. Relative angular rates between the OBS and RSO are recorded to ensure slew rates are not too large.

The method for calculating each metric is described in the following sections. Most of the metrics are based on those used in the methodology adapted from Ref. 5, and are further referenced in Space Mission Analysis and Design (SMAD). [11] Yates and Spanbauer used an iteration based simulation model for their analysis, so some of the calculations had to be modified from versions used in their research. For scenarios where there was no contact between the RSOs and the OBSs, such as cases where the semi major axes are greater than the range constraint, default metrics are used. Percent Coverage is calculated as $0 \%$, Mean Duration is calculated as $0 \mathrm{sec}$, and Time Average Gap and Mean Response Time are both calculated as the total simulation time.

\subsection{Percent Coverage (PC)}

Percent Coverage is the percentage of total simulation time that each RSO is in contact with at least 1 OBS. Higher values for percent coverage are associated with a stronger constellation performance, but can be associated with lower revisit rates. Equation 9 includes the AccessDuration set, which is an array of each conjunction duration in order or occurrence. 


$$
P C=\frac{\sum(\text { AccessDuration })}{\text { TotalSimTime }}
$$

\subsection{Time Average Gap (TAG)}

Time average gap is "the average length of the gap we would find if we randomly sampled the system".[11] It's calculated by assembling an array of all the coverage gap durations, and then taking the sum of the squares. The sum of the squares value is then divided by the total simulation time to produce an average time gap metric.

$$
T A G=\frac{\sum\left(\text { DurationGaps }^{2}\right)}{\text { TotalSimTime }}
$$

\subsection{Mean Duration (MD)}

The mean duration is a simple calculation that captures a different aspect of the duration intervals than percent coverage. An array of all the access intervals is averaged to find the mean length of access for each RSO. The value for each RSO is then averaged to get a single value to represent the mean duration for each constellation.

$$
M D=\frac{\sum \text { AccessDuration }}{\# \text { Durations }}
$$

\subsection{Mean Response Time (MRT)}

Two values are required for computing MRT, a response time counter and response summation. In a simulation based on timesteps, for each timestep that an RSO is not in contact with an OBS, the response summation is incremented by the full value of the current response time. For each 
conjunction gap, the response time counter keeps track of a growing average that weighs incremental time. For a simulation not based on timesteps, the nth triangle number is used to compute the summation of the current response time.

Mean response time focuses on how long it takes the observer satellites to reengage the RSO by incrementing a response time variable by the full duration of the gap every second. This is accomplished by using a numerical summation equation to solve for the response summation, and dividing by the full simulation time. The MRTsum is calculated by taking the sigma summation of each component of the DurationGaps array. Each gap interval has the nth triangle number computed and added to the MRTsum array, which is ultimately summed and divided by the total simulation time. Eq. (12) below is used to compute the MRTsum array, while Eq. (13) is used to calculate the final metric.

$$
\begin{gathered}
\sum_{N=1}^{K} N=\frac{K(K+1)}{2} \\
M R T=\frac{\sum M R T \text { sum }}{\text { TotalSimTime }}
\end{gathered}
$$

\subsection{Metric Rollup}

To directly compare and contrast the performances of the different constellations, a method of generating a single, succinct score was developed that encapsulates the performance of a constellation in each metric across all 3 Tiers. The total score represents the average performance of every RSO in the constellation with respect to all four grading metrics.

Within each Tier, the results for each metric are normalized by the best score, and then summed for each constellation for a best possible score of 4 . A 4 is achieved by a single case having the 
best performance in all four metrics. The scores for each Tier are then averaged together for a single rating for each constellation design. Every case analyzed for a cluster is normalized to the same values, making the scoring consistent across each category of cases. Figure 11 illustrates the metric rollup process. The normalization process occurs uniformly across all constellation types within a cluster, but is done independently for each cluster. Table 3 in Section IV.A shows the results for Cluster 1 circular cases, and showcases the metric rollup used as a comparison tool.

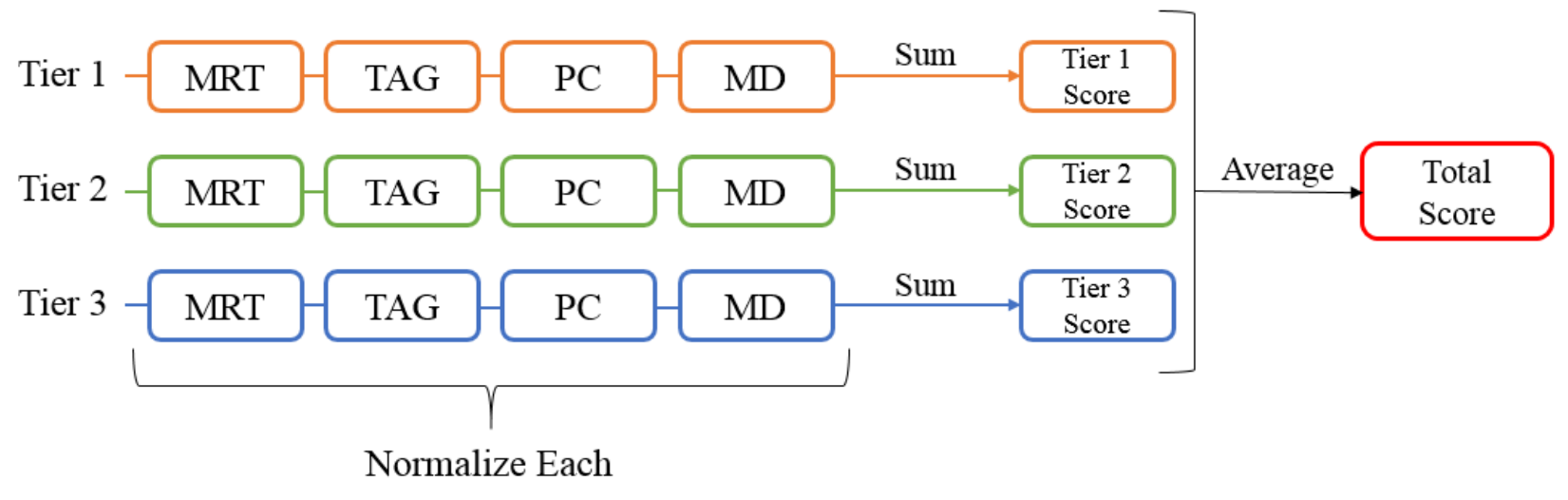

Fig. 11 Metric Rollup Flowchart

The scoring is intended to make the comparison process objective and enable a fair judgement to be made across all considered constellations. There are many different factors that go into deciding if a constellation design will be effective, and reducing each designs' performance into a single score is intended to provide an objective way to determine the optimal design. A well performing constellation based on these metrics is certain to provide adequate coverage based on the mission's scope. A constellation that is successful in only one Tier is unlikely to have a favorable final score, just as a constellation that performs great in a single metric but poorly in other metrics is also unable to achieve a relatively high final score. 


\section{Analysis and Results}

This research focuses on comparing and contrasting constellation designs for the purpose of orbital debris mitigation through LDR and SSA observation. The evaluation breaks the analysis into three maximum-range Tiers representing $100 \mathrm{~km}, 600 \mathrm{~km}$, and $2400 \mathrm{~km}$. Each constellation has a separate simulation run for each Tier, with different set conjunction constraints. Results from each Tier are normalized and combined into a single value that represents the performance of each case, with each Tier having equal impact on the total score. The principal metrics of interest used to evaluate the different constellation designs are MRT, TAG, PC, and MD. Color coding is used in all tables and graphics to make comparison easier, with Tier 1 results shown in orange, Tier 2 results shown in green, and Tier 3 results shown in blue.

It was hypothesized that ideal LDR constellation designs would have different orbital parameters for each RSO cluster, given the differences in inclination and altitude. All three clusters, however, produced remarkably similar results, with the best performing case for each being the non-maneuvering, circular, $-50 \mathrm{~km}$ offset constellation. The results also show the same trends in metrics and constellation performance, with mostly symmetrical behavior. Based on the similarity between clusters in static cases, maneuvering cases were only analyzed for Cluster 1 .

\section{A. Static Circular Cases}

The first set of constellations evaluated were static, circular orbits with varying offsets from the central altitudes of each cluster. All constellations evaluated contain eight satellites evenly distributed around the Earth by RAAN. Figures 12-14 depict the total score of each case for each cluster, providing a summary of the performance of each constellation. Tables 3-5 show the full performance results for each cluster, while Figures 15-17 plot each of the metrics at all three Tiers. Tables 3-5 display all metrics for each case, and include a cluster conjunction (CC) percentage 
which represents the percentage of RSOs that had a conjunction. It was determined early on in the analysis that any constellations with offsets greater than the Tier 2 maximum conjunction distance perform significantly worse than other cases. As a result, the range of cases analyzed includes a large distribution of altitudes between $-550 \mathrm{~km}$ and $+550 \mathrm{~km}$. Offsets greater than the Tier 1 maximum conjunction distance also perform worse overall, since they engage in no conjunctions in Tier 1 . All of the best performing circular scenarios have altitude offsets less than $100 \mathrm{~km}$, but including larger altitude offset cases is important for comparing how the metrics improved with higher Tiers.

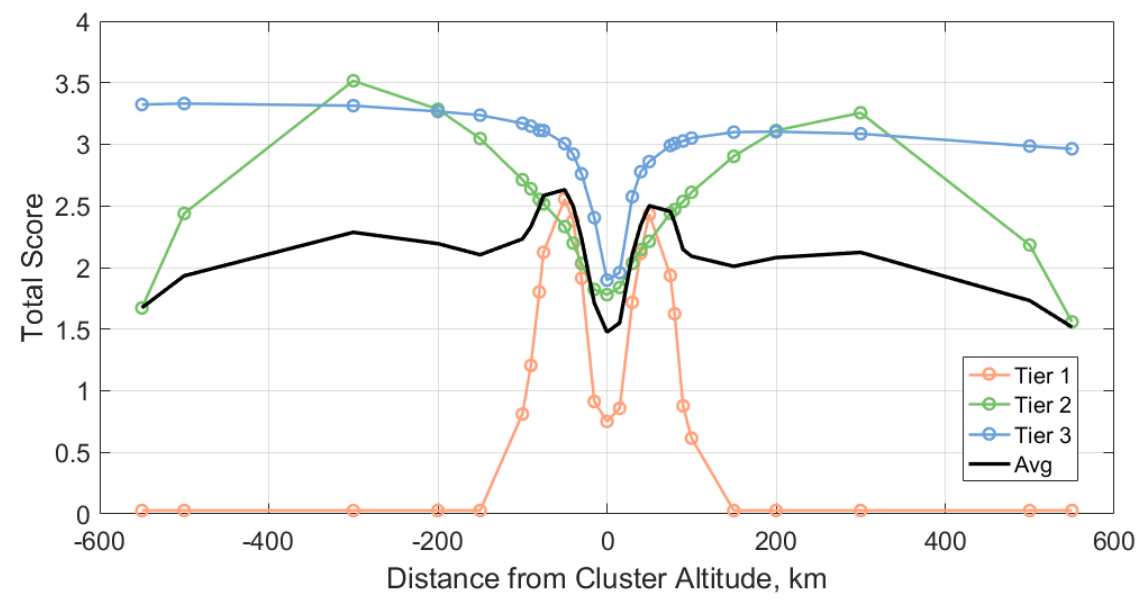

Fig. 12 Cluster 1 Circular Static Total Score

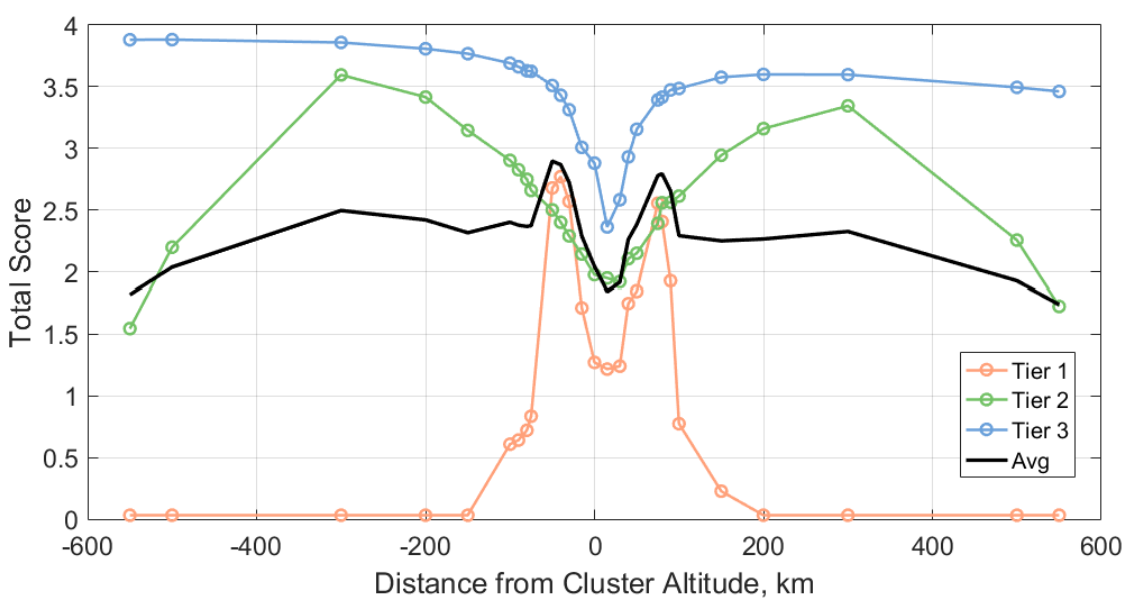

Fig. 13 Cluster 2 Circular Static Total Score 


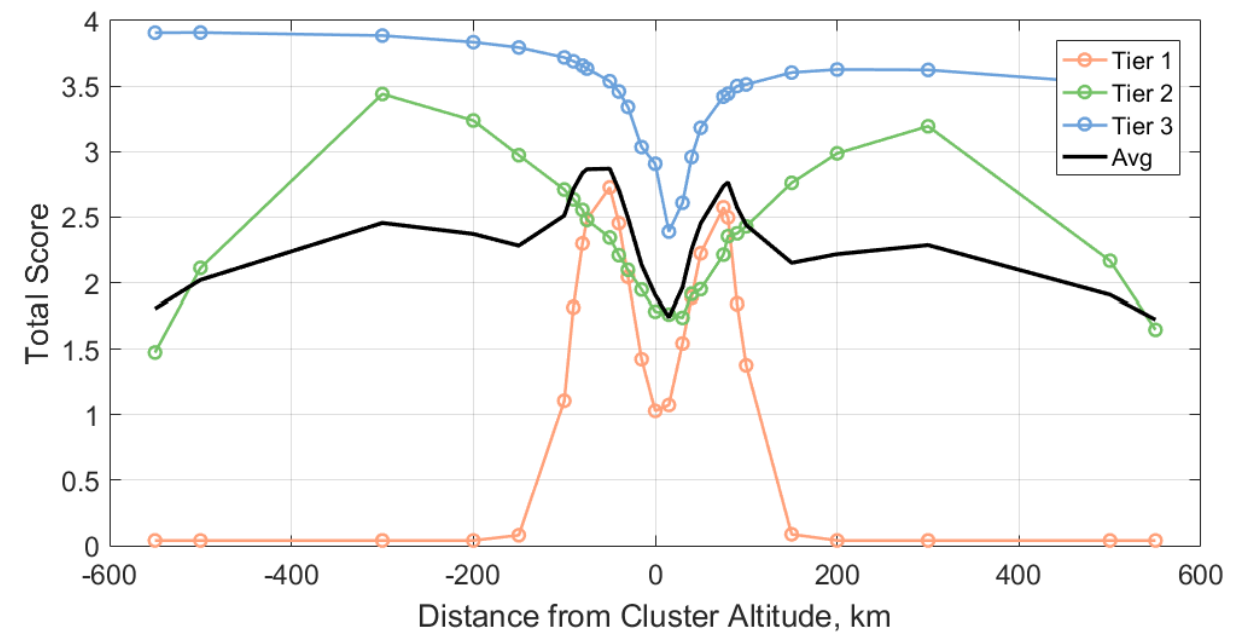

Fig. 14 Cluster 3 Circular Static Total Score

The relationships are roughly symmetric for all three clusters, with peaks in constellation performance at $50 \%$ of maximum range for Tiers 1 and 2, and fairly steady performance in Tier 3 . Cases with larger offsets perform well in Tier 3, but as expected provide no capability at the lower Tier levels. Also as expected, higher Tiers provide exceptionally better performance for every case examined as this results in longer conjunction intervals.

Almost all cases perform well in Tier 3, with MRTs and TAGs under an hour and PCs of about 14\%. Tier 3 also shows an average conjunction duration of almost 9 minutes. The Tier 2 metrics have much more variation in the performance of each case, with the $\pm 300 \mathrm{~km}$ cases performing the best. Constellations centered exactly at the debris cluster altitude perform the worst. This is expected since no altitude offset means a lower drift rate and less relative movement between the OBSs and RSOs within the cluster, leading to less interaction between the satellites. The best performing cases within Tier 2 have MRTs and TAGs around 5 hours and PCs under 2\%. Tier 1 metrics show the most variation in performance as well as the worst performing metrics. All MDs were lower than 1 minute, but the best performing cases in Tier 1 still have favorable MRTs and TAGs of under 2 days. 
Table 3 Cluster 1 Circular Static Performance Results

\begin{tabular}{|c|c|c|c|c|c|c|c|c|c|c|c|c|c|c|c|c|c|c|c|}
\hline \multirow[b]{2}{*}{ Case } & \multicolumn{6}{|c|}{ Tier 1: $100 \mathrm{~km}$} & \multicolumn{6}{|c|}{ Tier 2: $600 \mathrm{~km}$} & \multicolumn{6}{|c|}{ Tier 3: $2400 \mathrm{~km}$} & \multirow{2}{*}{$\begin{array}{l}\text { Total } \\
\text { Score }\end{array}$} \\
\hline & $\begin{array}{c}\text { MRT } \\
\text { (days) }\end{array}$ & $\begin{array}{l}\text { TAG } \\
\text { (days) }\end{array}$ & PC (\%) & $\begin{array}{c}\text { MeanD } \\
(\mathrm{min})\end{array}$ & CC (\%) & Score & $\begin{array}{l}\text { MRT } \\
\text { (days) }\end{array}$ & $\begin{array}{c}\text { TAG } \\
\text { (days) }\end{array}$ & PC (\%) & $\begin{array}{c}\text { MeanD } \\
(\mathrm{min})\end{array}$ & CC (\%) & Score & $\begin{array}{l}\text { MRT } \\
\text { (days) }\end{array}$ & $\begin{array}{c}\text { TAG } \\
\text { (days) }\end{array}$ & PC (\%) & $\begin{array}{c}\text { MeanD } \\
(\mathrm{min})\end{array}$ & $\mathrm{CC}(\%)$ & Score & \\
\hline circ $+550 \mathrm{~km}$ 8obj 0 Man & 90.00 & 90.00 & 0.00 & 0.00 & 0 & 0.028 & 0.306 & 0.523 & 0.685 & 1.243 & 100 & 1.559 & 0.025 & 0.048 & 12.511 & 8.503 & 100 & 2.964 & 1.517 \\
\hline circ $+500 \mathrm{~km} 8$ obj $0 \mathrm{Man}$ & 90.00 & 90.00 & 0.00 & 0.00 & 0 & 0.028 & 0.194 & 0.353 & 0.777 & 1.671 & 100 & 2.183 & 0.025 & 0.048 & 12.717 & 8.543 & 100 & 2.986 & 1.732 \\
\hline circ $+300 \mathrm{~km} 8$ obj 0Man & 90.00 & 90.00 & 0.00 & 0.00 & 0 & 0.028 & 0.137 & 0.256 & 1.414 & 2.510 & 100 & 3.256 & 0.024 & 0.046 & 13.375 & 8.662 & 100 & 3.086 & 2.123 \\
\hline circ $+200 \mathrm{~km}$ 8obj 0Man & 90.00 & 90.00 & 0.00 & 0.00 & 0 & 0.028 & 0.166 & 0.310 & 1.621 & 2.715 & 100 & 3.112 & 0.024 & 0.046 & 13.703 & 8.690 & 100 & 3.103 & 2.081 \\
\hline circ $+150 \mathrm{~km}$ 8obj 0Man & 90.00 & 90.00 & 0.00 & 0.00 & 0 & 0.028 & 0.203 & 0.378 & 1.692 & 2.788 & 100 & 2.903 & 0.024 & 0.047 & 13.865 & 8.697 & 100 & 3.099 & 2.010 \\
\hline circ $+100 \mathrm{~km} 8$ obj $0 \mathrm{Man}$ & 14.163 & 18.391 & 5.323 & 0.256 & 100 & 0.615 & 0.278 & 0.522 & 1.742 & 2.831 & 100 & 2.611 & 0.025 & 0.048 & 14.035 & 8.696 & 100 & 3.051 & 2.092 \\
\hline circ $+90 \mathrm{~km} 8 \mathrm{obj}$ 0Man & 4.539 & 6.704 & 3.485 & 0.305 & 100 & 0.878 & 0.305 & 0.571 & 1.737 & 2.843 & 100 & 2.537 & 0.026 & 0.049 & 14.095 & 8.706 & 100 & 3.027 & 2.147 \\
\hline circ $+80 \mathrm{~km}$ 8obj 0Man & 1.622 & 2.658 & 1.565 & 0.404 & 100 & 1.625 & 0.338 & 0.634 & 1.769 & 2.837 & 100 & 2.471 & 0.026 & 0.050 & 14.083 & 8.705 & 100 & 3.007 & 2.368 \\
\hline circ $+75 \mathrm{~km}$ 8obj 0Man & 1.286 & 2.121 & 1.465 & 0.417 & 100 & 1.935 & 0.358 & 0.672 & 1.781 & 2.852 & 100 & 2.441 & 0.026 & 0.050 & 14.140 & 8.705 & 100 & 2.990 & 2.455 \\
\hline circ $+50 \mathrm{~km} 8 \mathrm{obj} 0 \mathrm{Man}$ & 0.970 & 1.772 & 0.895 & 0.558 & 100 & 2.432 & 0.528 & 0.998 & 1.761 & 2.856 & 100 & 2.213 & 0.029 & 0.055 & 14.224 & 8.710 & 100 & 2.860 & 2.502 \\
\hline circ $+40 \mathrm{~km}$ 8obj 0Man & 1.182 & 2.141 & 0.450 & 0.587 & 100 & 2.116 & 0.681 & 1.267 & 1.812 & 2.883 & 100 & 2.145 & 0.031 & 0.058 & 14.184 & 8.686 & 100 & 2.778 & 2.346 \\
\hline circ $+30 \mathrm{~km} 8$ obj 0Man & 1.620 & 2.984 & 0.216 & 0.622 & 100 & 1.718 & 0.944 & 1.806 & 1.794 & 2.890 & 100 & 2.035 & 0.036 & 0.069 & 14.231 & 8.723 & 100 & 2.575 & 2.109 \\
\hline circ $+15 \mathrm{~km}$ 8obj 0Man & 9.412 & 9.455 & 0.214 & 0.599 & 100 & 0.857 & 5.926 & 6.028 & 1.816 & 2.859 & 100 & 1.838 & 0.092 & 0.156 & 14.542 & 8.709 & 100 & 1.959 & 1.551 \\
\hline circ +0km 8obj OMan & 15.629 & 15.190 & 0.130 & 0.599 & 100 & 0.751 & 6.283 & 9.027 & 1.848 & 2.680 & 100 & 1.780 & 0.088 & 0.157 & 14.123 & 8.204 & 100 & 1.898 & 1.476 \\
\hline circ - $15 \mathrm{~km}$ 8obj 0Man & 11.969 & 6.172 & 0.207 & 0.588 & 100 & 0.913 & 9.711 & 3.310 & 1.750 & 2.833 & 100 & 1.822 & 0.047 & 0.073 & 14.174 & 8.546 & 100 & 2.405 & 1.713 \\
\hline circ -30km 8obj 0Man & 1.426 & 2.424 & 0.452 & 0.606 & 100 & 1.914 & 1.117 & 1.424 & 1.793 & 2.844 & 100 & 2.033 & 0.032 & 0.057 & 14.288 & 8.677 & 100 & 2.760 & 2.235 \\
\hline circ $-40 \mathrm{~km}$ 8obj 0Man & 1.022 & 1.819 & 0.732 & 0.587 & 100 & 2.382 & 0.566 & 1.057 & 1.807 & 2.832 & 100 & 2.198 & 0.028 & 0.053 & 14.355 & 8.651 & 100 & 2.920 & 2.500 \\
\hline circ - $50 \mathrm{~km}$ 8obj 0Man & 0.938 & 1.573 & 0.905 & 0.534 & 100 & 2.556 & 0.454 & 0.857 & 1.865 & 2.849 & 100 & 2.333 & 0.026 & 0.050 & 14.408 & 8.669 & 100 & 3.006 & 2.632 \\
\hline circ $-75 \mathrm{~km}$ 8obj 0Man & 1.169 & 1.865 & 1.204 & 0.443 & 100 & 2.124 & 0.319 & 0.600 & 1.787 & 2.826 & 100 & 2.517 & 0.024 & 0.047 & 14.334 & 8.637 & 100 & 3.111 & 2.584 \\
\hline circ -80km 8obj 0Man & 1.448 & 2.244 & 1.638 & 0.399 & 100 & 1.802 & 0.305 & 0.569 & 1.787 & 2.817 & 100 & 2.553 & 0.024 & 0.046 & 14.328 & 8.634 & 100 & 3.114 & 2.490 \\
\hline circ $-90 \mathrm{~km}$ 8obj OMan & 2.638 & 4.063 & 2.871 & 0.358 & 100 & 1.206 & 0.274 & 0.515 & 1.788 & 2.815 & 100 & 2.639 & 0.024 & 0.046 & 14.377 & 8.635 & 100 & 3.148 & 2.331 \\
\hline circ $-100 \mathrm{~km}$ 8obj 0Man & 6.758 & 10.719 & 6.222 & 0.282 & 100 & 0.811 & 0.250 & 0.472 & 1.778 & 2.799 & 100 & 2.713 & 0.023 & 0.045 & $\mid 14.377$ & 8.624 & 100 & 3.171 & 2.232 \\
\hline circ $-150 \mathrm{~km}$ 8obj 0Man & 90.00 & 90.00 & 0.00 & 0.00 & 0 & 0.028 & 0.184 & 0.343 & 1.769 & 2.747 & 100 & 3.047 & 0.022 & 0.043 & 14.364 & 8.576 & 100 & 3.237 & 2.104 \\
\hline circ-200km 8obj 0Man & 90.00 & 90.00 & 0.00 & 0.00 & 0 & 0.028 & 0.150 & 0.280 & 1.699 & 2.656 & 100 & 3.285 & 0.022 & 0.042 & 14.324 & 8.533 & 100 & 3.267 & 2.194 \\
\hline circ-300km 8obj 0Man & 90.00 & 90.00 & 0.00 & 0.00 & 0 & 0.028 & 0.123 & 0.226 & 1.533 & 2.454 & 100 & 3.516 & 0.021 & 0.041 & 14.294 & 8.426 & 100 & 3.314 & 2.286 \\
\hline circ $-500 \mathrm{~km}$ 8obj 0Man & 90.00 & 90.00 & 0.00 & 0.00 & 0 & 0.028 & 0.161 & 0.288 & 0.779 & 1.616 & 100 & 2.439 & 0.021 & 0.040 & 14.090 & 8.160 & 100 & 3.331 & 1.933 \\
\hline circ $-550 \mathrm{~km}$ 8obj 0Man & 90.00 & 90.00 & 0.00 & 0.00 & 0 & 0.028 & 0.253 & 0.426 & 0.550 & 1.218 & 100 & 1.672 & 0.021 & 0.039 & 13.964 & 8.073 & 100 & 3.323 & 1.674 \\
\hline
\end{tabular}

Table 4 Cluster 2 Circular Static Performance Results

\begin{tabular}{|c|c|c|c|c|c|c|c|c|c|c|c|c|c|c|c|c|c|c|c|}
\hline \multirow[b]{2}{*}{ Case } & \multicolumn{6}{|c|}{ Tier 1: $100 \mathrm{~km}$} & \multicolumn{6}{|c|}{ Tier 2: $600 \mathrm{~km}$} & \multicolumn{6}{|c|}{ Tier 3: $2400 \mathrm{~km}$} & \multirow{2}{*}{$\begin{array}{l}\text { Total } \\
\text { Score }\end{array}$} \\
\hline & $\begin{array}{c}\text { MRT } \\
\text { (days) }\end{array}$ & $\begin{array}{c}\text { TAG } \\
\text { (days) }\end{array}$ & PC (\%) & $\begin{array}{c}\text { MeanD } \\
(\mathrm{min})\end{array}$ & CC (\%) & Score & $\begin{array}{l}\text { MRT } \\
\text { (days) }\end{array}$ & $\begin{array}{c}\text { TAG } \\
\text { (days) }\end{array}$ & PC (\%) & $\begin{array}{c}\text { MeanD } \\
(\min )\end{array}$ & $\mathrm{CC}(\%)$ & Score & $\begin{array}{c}\text { MRT } \\
\text { (days) }\end{array}$ & $\begin{array}{c}\text { TAG } \\
\text { (days) }\end{array}$ & PC (\%) & $\begin{array}{c}\text { MeanD } \\
(\min )\end{array}$ & CC (\%) & Score & \\
\hline circ $+550 \mathrm{~km}$ 8obj 0Man & 90.00 & 90.00 & 0.00 & 0.00 & 0 & 0.033 & 0.300 & 0.547 & 0.628 & 1.148 & 100 & 1.726 & 0.026 & 0.049 & 12.287 & 8.194 & 100 & 3.461 & 1.740 \\
\hline circ $+500 \mathrm{~km}$ 8obj 0Man & 90.00 & 90.00 & 0.00 & 0.00 & 0 & 0.033 & 0.215 & 0.395 & 0.712 & 1.475 & 100 & 2.255 & 0.025 & 0.048 & 12.431 & 8.230 & 100 & 3.494 & 1.927 \\
\hline circ $+300 \mathrm{~km}$ 8obj 0Man & 90.00 & 90.00 & 0.00 & 0.00 & 0 & 0.033 & 0.152 & 0.279 & 1.212 & 2.159 & 100 & 3.344 & 0.025 & 0.047 & 12.999 & 8.347 & 100 & 3.597 & 2.325 \\
\hline circ $+200 \mathrm{~km}$ 8obj 0Man & 90.00 & 90.00 & 0.00 & 0.00 & 0 & 0.033 & 0.194 & 0.352 & 1.397 & 2.337 & 100 & 3.159 & 0.025 & 0.047 & 13.262 & 8.370 & 100 & 3.599 & 2.264 \\
\hline circ $+150 \mathrm{~km}$ 8obj 0Man & 76.353 & 79.441 & 2.354 & 0.019 & 100 & 0.228 & 0.241 & 0.442 & 1.458 & 2.390 & 100 & 2.943 & 0.025 & 0.048 & 13.425 & 8.376 & 100 & 3.576 & 2.249 \\
\hline circ $+100 \mathrm{~km}$ 8obj 0Man & 8.790 & 11.512 & 0.691 & 0.240 & 100 & 0.775 & 0.356 & 0.648 & 1.473 & 2.418 & 100 & 2.612 & 0.027 & 0.051 & 13.469 & 8.370 & 100 & 3.485 & 2.291 \\
\hline circ +90km 8obj 0Man & 1.689 & 2.698 & 0.435 & 0.300 & 100 & 1.927 & 0.392 & 0.723 & 1.498 & 2.438 & 100 & 2.562 & 0.027 & 0.052 & 13.578 & 8.382 & 100 & 3.473 & 2.654 \\
\hline circ $+80 \mathrm{~km}$ 8obj 0Man & 1.286 & 2.086 & 0.318 & 0.343 & 100 & 2.406 & 0.453 & 0.827 & 1.636 & 2.436 & 100 & 2.558 & 0.028 & 0.054 & 13.569 & 8.373 & 100 & 3.414 & 2.793 \\
\hline circ $+75 \mathrm{~km}$ 8obj 0Man & 1.217 & 1.989 & 0.248 & 0.376 & 100 & 2.554 & 0.479 & 0.901 & 1.429 & 2.435 & 100 & 2.391 & 0.029 & 0.055 & 13.575 & 8.376 & 100 & 3.392 & 2.779 \\
\hline circ $+50 \mathrm{~km} 8 \mathrm{obj} 0 \mathrm{Man}$ & 2.097 & 3.623 & 0.146 & 0.431 & 100 & 1.845 & 1.305 & 2.399 & 1.587 & 2.447 & 100 & 2.149 & 0.035 & 0.065 & 13.598 & 8.363 & 100 & 3.154 & 2.382 \\
\hline circ $+40 \mathrm{~km}$ 8obj 0Man & 2.385 & 4.506 & 0.126 & 0.469 & 100 & 1.748 & 1.320 & 2.479 & 1.517 & 2.455 & 100 & 2.105 & 0.043 & 0.083 & 13.803 & 8.418 & 100 & 2.930 & 2.261 \\
\hline circ $+30 \mathrm{~km}$ 8obj 0Man & 7.255 & 9.050 & 0.094 & 0.476 & 100 & 1.242 & 3.680 & 5.258 & 1.455 & 2.377 & 100 & 1.920 & 0.059 & 0.111 & 13.059 & 8.097 & 100 & 2.582 & 1.915 \\
\hline circ $+15 \mathrm{~km} 8 \mathrm{obj}$ OMan & 13.230 & 14.421 & 0.070 & 0.543 & 100 & 1.218 & 7.516 & 8.676 & 1.498 & 2.475 & 100 & 1.949 & 0.113 & 0.147 & 13.466 & 8.136 & 100 & 2.362 & 1.843 \\
\hline circ $+0 \mathrm{~km}$ 8obj 0Man & 6.323 & 8.659 & 0.084 & 0.476 & 100 & 1.272 & 4.599 & 4.583 & 1.525 & 2.411 & 100 & 1.976 & 0.046 & 0.080 & 13.629 & 8.223 & 100 & 2.879 & 2.042 \\
\hline circ -15km 8obj 0Man & 2.566 & 4.426 & 0.139 & 0.463 & 100 & 1.713 & 1.160 & 2.168 & 1.557 & 2.417 & 100 & 2.142 & 0.040 & 0.075 & 13.733 & 8.331 & 100 & 3.007 & 2.287 \\
\hline circ -30km 8obj 0Man & 1.233 & 2.177 & 0.275 & 0.434 & 100 & 2.569 & 0.679 & 1.251 & 1.534 & 2.411 & 100 & 2.289 & 0.031 & 0.059 & 13.852 & 8.367 & 100 & 3.312 & 2.723 \\
\hline circ-40km 8obj 0Man & 1.090 & 1.891 & 0.277 & 0.408 & 100 & 2.770 & 0.539 & 0.991 & 1.537 & 2.431 & 100 & 2.400 & 0.028 & 0.054 & 13.740 & 8.324 & 100 & 3.430 & 2.867 \\
\hline circ -50km 8obj 0Man & 1.157 & 1.937 & 0.310 & 0.402 & 100 & 2.678 & 0.450 & 0.826 & 1.547 & 2.422 & 100 & 2.500 & 0.027 & 0.051 & 13.818 & 8.343 & 100 & 3.509 & 2.896 \\
\hline circ $-75 \mathrm{~km}$ 8obj 0Man & 12.530 & 14.262 & 0.907 & 0.302 & 100 & 0.836 & 0.322 & 0.607 & 1.451 & 2.408 & 100 & 2.658 & 0.025 & 0.048 & 13.831 & 8.325 & 100 & 3.624 & 2.373 \\
\hline circ -80km 8obj 0Man & 17.990 & 19.816 & 0.692 & 0.282 & 100 & 0.722 & 0.314 & 0.575 & 1.546 & 2.405 & 100 & 2.748 & 0.025 & 0.047 & 13.800 & 8.316 & 100 & 3.628 & 2.366 \\
\hline circ -90km 8obj 0Man & 27.874 & 32.555 & 1.664 & 0.236 & 100 & 0.642 & 0.286 & 0.525 & 1.536 & 2.411 & 100 & 2.826 & 0.024 & 0.047 & 13.838 & 8.322 & 100 & 3.661 & 2.376 \\
\hline circ -100km 8obj 0Man & 71.509 & 85.902 & 3.717 & 0.178 & 100 & 0.609 & 0.261 & 0.485 & 1.541 & 2.386 & 100 & 2.902 & 0.024 & 0.046 & 13.833 & 8.311 & 100 & 3.690 & 2.400 \\
\hline circ $-150 \mathrm{~km}$ 8obj 0Man & 90.00 & 90.00 & 0.00 & 0.00 & 0 & 0.033 & 0.191 & 0.362 & 1.396 & 2.329 & 100 & 3.145 & 0.023 & 0.044 & 13.845 & 8.271 & 100 & 3.767 & 2.315 \\
\hline circ -200km 8obj 0Man & 90.00 & 90.00 & 0.00 & 0.00 & 0 & 0.033 & 0.161 & 0.297 & 1.430 & 2.265 & 100 & 3.415 & 0.022 & 0.043 & 13.815 & 8.225 & 100 & 3.807 & 2.419 \\
\hline circ-300km 8obj 0Man & 90.00 & 90.00 & 0.00 & 0.00 & 0 & 0.033 & 0.132 & 0.243 & 1.255 & 2.074 & 100 & 3.595 & 0.022 & 0.041 & 13.824 & 8.118 & 100 & 3.858 & 2.495 \\
\hline circ-500km 8obj 0Man & 90.00 & 90.00 & 0.00 & 0.00 & 0 & 0.033 & 0.225 & 0.359 & 0.680 & 1.292 & 100 & 2.197 & 0.021 & 0.040 & 13.750 & 7.873 & 100 & 3.881 & 2.037 \\
\hline circ -550km 8obj 0Man & 90.00 & 90.00 & 0.00 & 0.00 & 0 & 0.033 & 0.335 & 0.559 & 0.570 & 0.924 & 100 & 1.546 & 0.021 & 0.040 & 13.661 & 7.800 & 100 & 3.880 & 1.820 \\
\hline
\end{tabular}


Table 5 Cluster 3 Circular Static Performance Results

\begin{tabular}{|c|c|c|c|c|c|c|c|c|c|c|c|c|c|c|c|c|c|c|c|}
\hline & \multicolumn{6}{|c|}{ Tier 1: $100 \mathrm{~km}$} & \multicolumn{6}{|c|}{ Tier 2: $600 \mathrm{~km}$} & \multicolumn{6}{|c|}{ Tier 3: $2400 \mathrm{~km}$} & \multirow{2}{*}{$\begin{array}{l}\text { Total } \\
\text { Score }\end{array}$} \\
\hline Case & $\begin{array}{c}\text { MRT } \\
\text { (days) }\end{array}$ & $\begin{array}{l}\text { TAG } \\
\text { (days) }\end{array}$ & PC (\%) & $\begin{array}{c}\text { MeanD } \\
(\mathrm{min})\end{array}$ & CC (\%) & Score & $\begin{array}{c}\text { MRT } \\
\text { (days) }\end{array}$ & $\begin{array}{l}\text { TAG } \\
\text { (days) }\end{array}$ & PC (\%) & \begin{tabular}{|c|} 
MeanD \\
$(\mathrm{min})$
\end{tabular} & CC (\%) & Score & $\begin{array}{c}\text { MRT } \\
\text { (days) }\end{array}$ & $\begin{array}{l}\text { TAG } \\
\text { (days) }\end{array}$ & PC (\%) & \begin{tabular}{|c|} 
MeanD \\
$(\mathrm{min})$
\end{tabular} & CC (\%) & Score & \\
\hline $\mathrm{circ}+550 \mathrm{~km}$ 8obj 0Man & 90.00 & 90.00 & 0.00 & 0.00 & 0 & 0.041 & 0.300 & 0.547 & 0.628 & 1.148 & 100 & 1.648 & 0.026 & 0.049 & 12.287 & 8.194 & 100 & 3.486 & 1.725 \\
\hline circ $+500 \mathrm{~km} 8$ obj $0 \mathrm{Man}$ & 90.00 & 90.00 & 0.00 & 0.00 & 0 & 0.041 & 0.215 & 0.395 & 0.712 & 1.475 & 100 & 2.167 & 0.025 & 0.048 & 12.431 & 8.230 & 100 & 3.519 & 1.909 \\
\hline circ $+300 \mathrm{~km} 8$ obj 0 Man & 90.00 & 90.00 & 0.00 & 0.00 & 0 & 0.041 & 0.152 & 0.279 & 1.212 & 2.159 & 100 & 3.193 & 0.025 & 0.047 & 12.999 & 8.347 & 100 & 3.623 & 2.286 \\
\hline circ $+200 \mathrm{~km}$ 8obj 0Man & 90.00 & 90.00 & 0.00 & 0.00 & 0 & .041 & 0.194 & 0.352 & 1.397 & 2.337 & 100 & 2.986 & 0.025 & 0.047 & 13.262 & 8.370 & 100 & 3.626 & 2.217 \\
\hline circ $+150 \mathrm{~km}$ 8obj 0Man & 88.979 & 89.028 & 0.658 & 0.005 & 91 & 088 & 0.241 & 0.442 & 1.458 & 2.390 & 100 & 2.761 & 0.025 & 0.048 & 13.425 & 8.376 & 100 & 3.603 & .151 \\
\hline circ $+100 \mathrm{~km}$ 8obj 0Man & 4.499 & 5.746 & 2.660 & 0.289 & 100 & 377 & 0.356 & 0.648 & 1.473 & 2.418 & 100 & 2.429 & 0.027 & 0.051 & 13.469 & 8.370 & 100 & 3.512 & .439 \\
\hline circ $+90 \mathrm{~km}$ 8obj 0Man & 2.560 & 3.556 & 1.508 & 0.313 & 100 & 842 & 0.392 & 0.723 & .498 & 2.438 & 10 & 376 & 0.027 & 0.052 & 13.578 & 8.382 & 100 & 00 & 73 \\
\hline $\mathrm{circ}+80 \mathrm{~km}$ 8obj 0Man & 1.615 & 2.499 & 0.970 & 0.360 & 100 & .497 & 0.453 & 0.827 & 1.636 & 2.436 & 100 & 2.354 & 0.028 & 0.054 & 3.569 & 8.373 & 100 & 3.441 & .764 \\
\hline circ $+75 \mathrm{~km}$ 8obj 0Man & 1.533 & 2.395 & 0.932 & 0.355 & 100 & 2.574 & 0.479 & 0.901 & 1.429 & 2.435 & 100 & 2.213 & 0.029 & 0.055 & 13.575 & 8.376 & 100 & 3.419 & 2.736 \\
\hline circ +50km 8obj 0Man & 1.914 & 3.352 & 0.276 & 0.436 & 100 & 2.224 & 1.305 & 2.399 & 1.587 & 2.447 & 100 & 1.951 & 0.035 & 0.065 & 13.598 & 8.363 & 100 & 3.181 & 2.452 \\
\hline circ $+40 \mathrm{~km}$ 8obj 0Man & 2.589 & 4.429 & 0.222 & 0.455 & 100 & & 1.320 & 2.479 & 1.517 & 2.455 & 10 & & 0.043 & 0.083 & 13.803 & 8.418 & 100 & & 2.255 \\
\hline circ $+30 \mathrm{~km}$ 8obj 0Man & 3.889 & 6.937 & 0.208 & 0.469 & 100 & .544 & 3.680 & 5.258 & 1.455 & 2.377 & 100 & 1.739 & 0.059 & 0.111 & 13.059 & 8.097 & 100 & 2.609 & 1.964 \\
\hline circ $+15 \mathrm{~km}$ 8obj 0Man & 25.010 & 22.323 & 0.208 & 0.510 & 10 & 1.075 & 7.516 & 8.676 & 1.498 & 2.475 & 10 & 1.763 & 0.113 & 0.147 & 13.466 & 8.136 & 100 & 2.389 & 1.742 \\
\hline circ +0km 8obj 0Man & 14.687 & 15.027 & 0.263 & 0.432 & 100 & 1.030 & 4.599 & 4.583 & 1.525 & 2.411 & 10 & 1.786 & 0.046 & 0.080 & 13.629 & 8.223 & 100 & 2.907 & 1.907 \\
\hline circ - $15 \mathrm{~km}$ 8obj 0Man & 7.178 & 5.792 & 0.155 & 0.465 & 100 & 1.424 & 1.160 & 2.168 & 1.557 & 2.417 & 100 & 1.948 & 0.040 & 0.075 & 13.733 & 8.331 & 100 & 3.034 & 2.136 \\
\hline circ -30km 8obj 0Man & 2.328 & 3.686 & 0.199 & 0.449 & 100 & 2.045 & 0.679 & 1.251 & 1.534 & 2.411 & 100 & 2.098 & 0.031 & 0.059 & 13.852 & 8.367 & 100 & 3.340 & 2.494 \\
\hline circ -40km 8obj 0Man & 1.695 & 2.765 & 0.217 & 0.432 & 100 & 2.454 & 0.539 & 0.991 & 1.537 & 2.431 & 100 & 2.209 & 0.028 & 0.054 & 13.740 & 8.324 & 100 & 3.458 & 2.707 \\
\hline circ - $50 \mathrm{~km}$ 8obj 0Man & 1.469 & 2.314 & 0.580 & 0.409 & 100 & 2.726 & 0.410 & 0.806 & 1.547 & 2.422 & 10 & & 0.027 & 0.051 & 13.818 & 8.343 & 100 & & 2.869 \\
\hline circ $-75 \mathrm{~km}$ 8obj 0Man & 1.625 & 2.496 & 1.051 & 0.352 & 10 & 2.484 & 0.322 & 0.607 & 1.451 & 2.408 & 100 & 2.478 & 0.025 & 0.049 & 13.811 & 8.325 & 100 & 3.633 & 2.865 \\
\hline circ -80km 8obj 0Man & 1.929 & 2.576 & 1.334 & 0.335 & 10 & 2.299 & 0.314 & 0.575 & 1.546 & 2.405 & 10 & 2.5 & 0.025 & 0.047 & 13.800 & 8.316 & 0 & 3.6 & 2.837 \\
\hline circ $-90 \mathrm{~km}$ 8obj 0Man & 2.710 & 3.748 & 2.508 & 0.303 & & 1.8 & 0.286 & 0.525 & 1.536 & 2.411 & 10 & & 0.024 & 0.047 & 13.838 & 8.322 & & & 2.713 \\
\hline circ $-100 \mathrm{~km}$ 8obj 0Man & 7.603 & 11.098 & 4.547 & 0.258 & 99.333 & 1.10 & 0.261 & 0.485 & 1.541 & 2.386 & 10 & 2.710 & 0.024 & 0.046 & 13.833 & 8.311 & 100 & 3.718 & 2.511 \\
\hline circ -150km 8obj 0Man & 87.091 & 87.374 & 0.540 & 0.004 & 99 & 0.081 & 0.191 & 0.362 & 1.396 & 2.329 & 100 & 2.972 & 0.023 & 0.044 & 13.845 & 8.271 & 100 & 3.794 & 2.282 \\
\hline circ -200km 8obj 0Man & 90.00 & 90.00 & 0.00 & 0.00 & 0 & 0.041 & 0.161 & 0.297 & 1.430 & 2.265 & 100 & 3.237 & 0.022 & 0.043 & 13.815 & 8.225 & 100 & 3.835 & 2.371 \\
\hline circ-300km 8obj 0Man & 90.00 & 90.00 & 0.00 & 0.00 & 0 & 0.041 & 0.132 & 0.243 & 1.255 & 2.074 & 100 & 3.439 & 0.022 & 0.041 & 13.824 & 8.118 & 100 & 3.885 & 2.455 \\
\hline circ $-500 \mathrm{~km}$ 8obj 0Man & 90.00 & 90.00 & 0.00 & 0.00 & 0 & 0.041 & 0.225 & 0.359 & 0.680 & 1.292 & 100 & 2.112 & 0.021 & 0.040 & 13.750 & 7.873 & 100 & 3.908 & 2.021 \\
\hline circ $-550 \mathrm{~km}$ 8obj 0Man & 90.00 & 90.00 & 0.00 & 0.00 & 0 & 0.041 & 0.335 & 0.559 & 0.570 & 0.924 & 100 & 1.475 & 0.021 & 0.040 & 13.661 & 7.800 & 100 & 3.907 & 1.808 \\
\hline
\end{tabular}
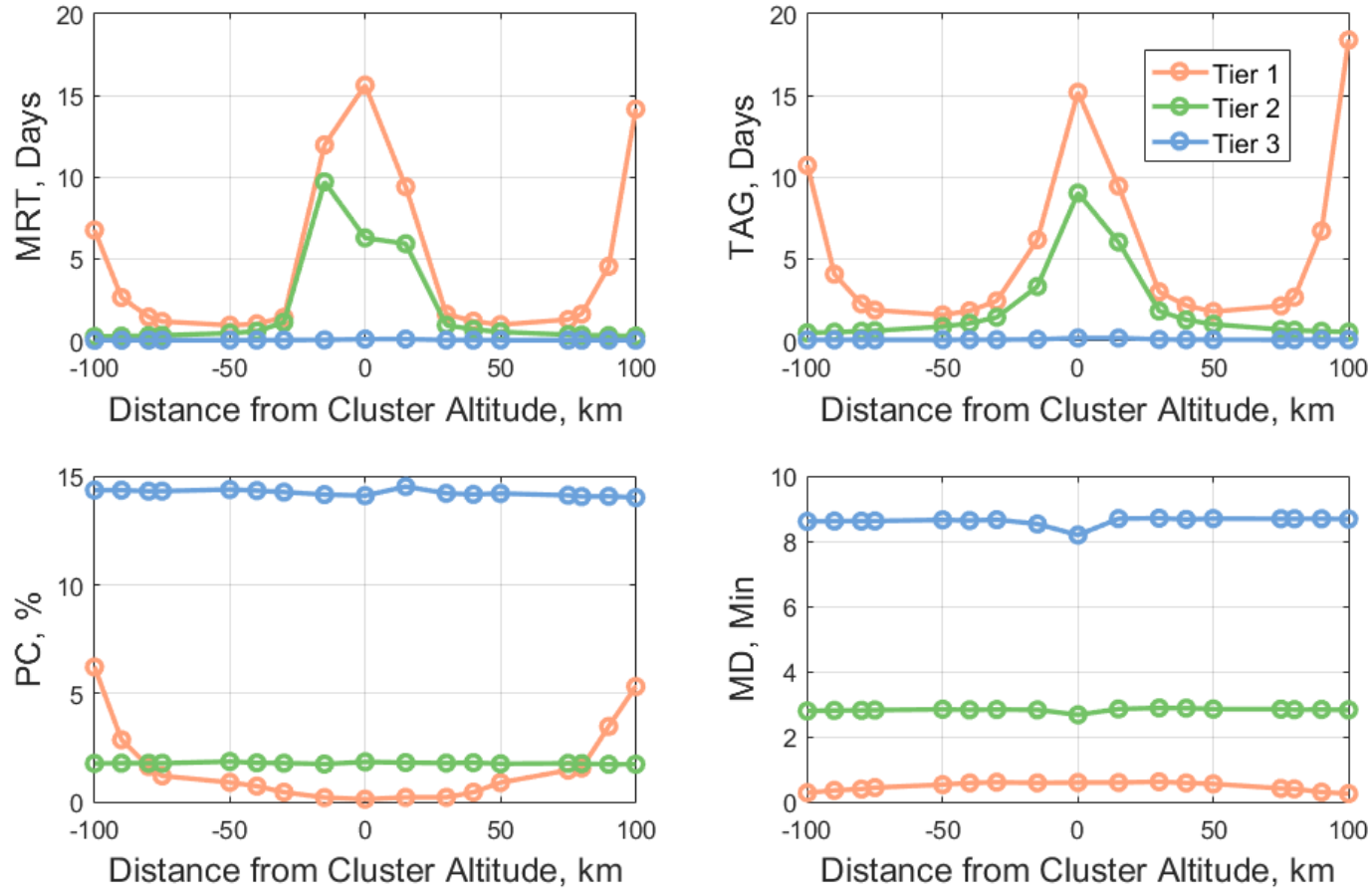

Fig. 15 Cluster 1 Circular Static Metrics 

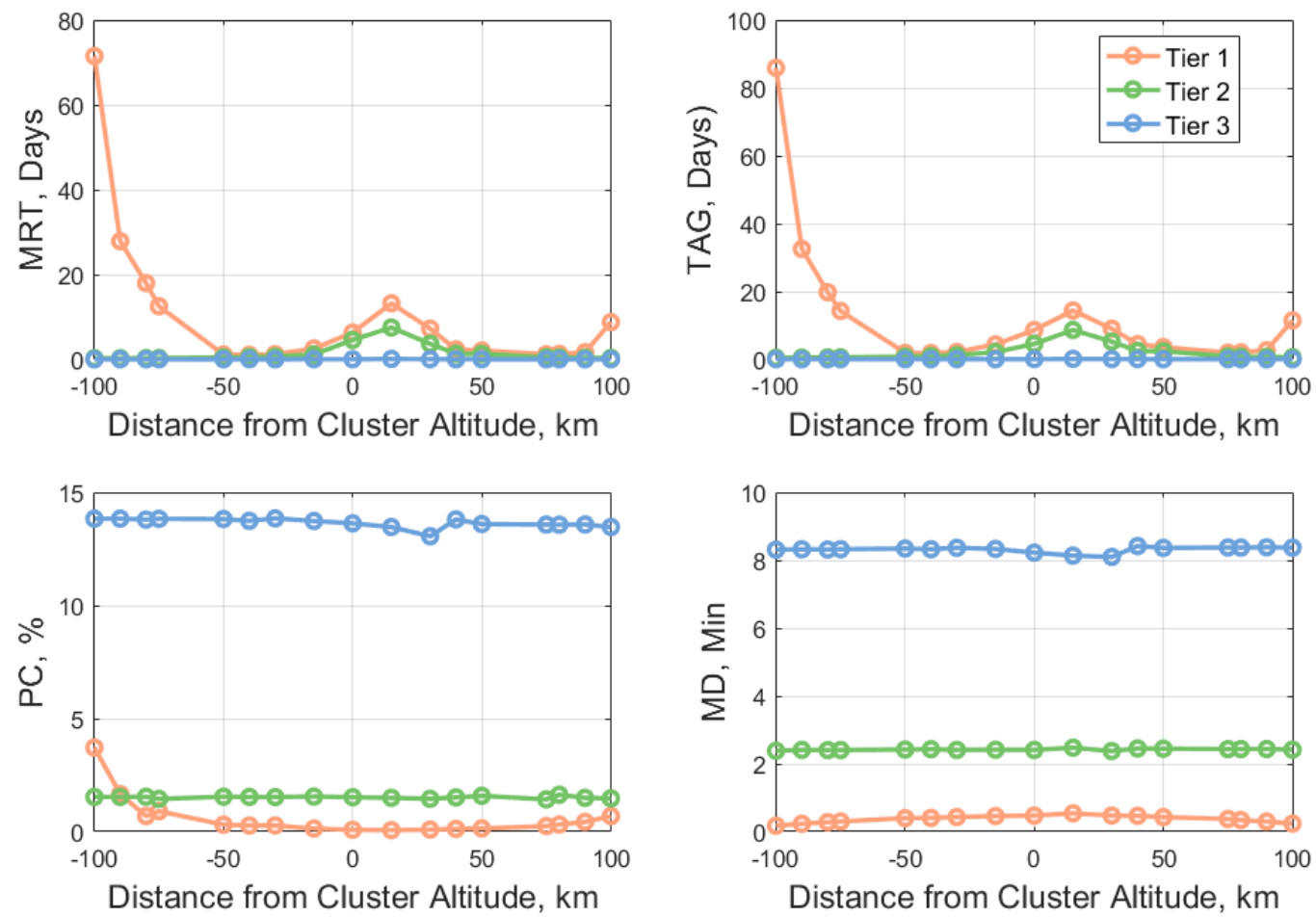

Fig. 16 Cluster 2 Circular Static Metrics
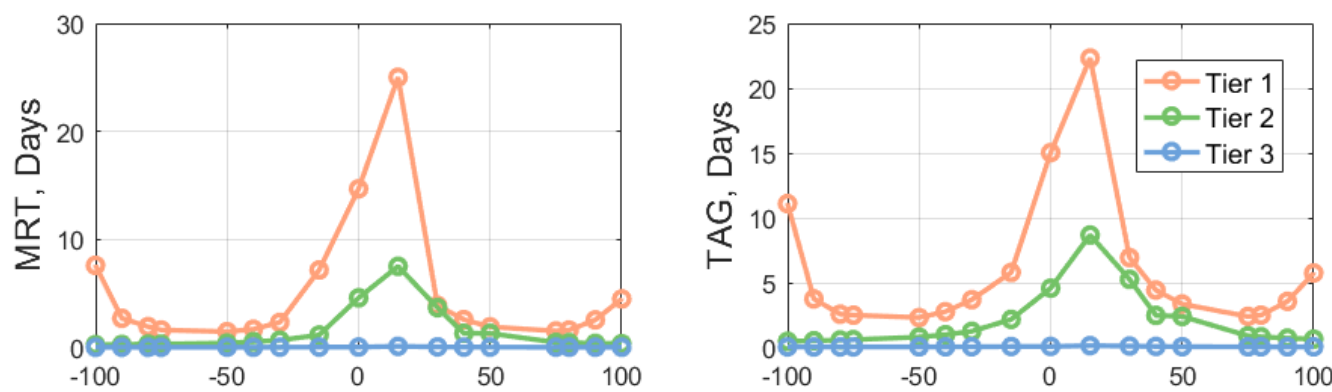

Distance from Cluster Altitude, $\mathrm{km}$
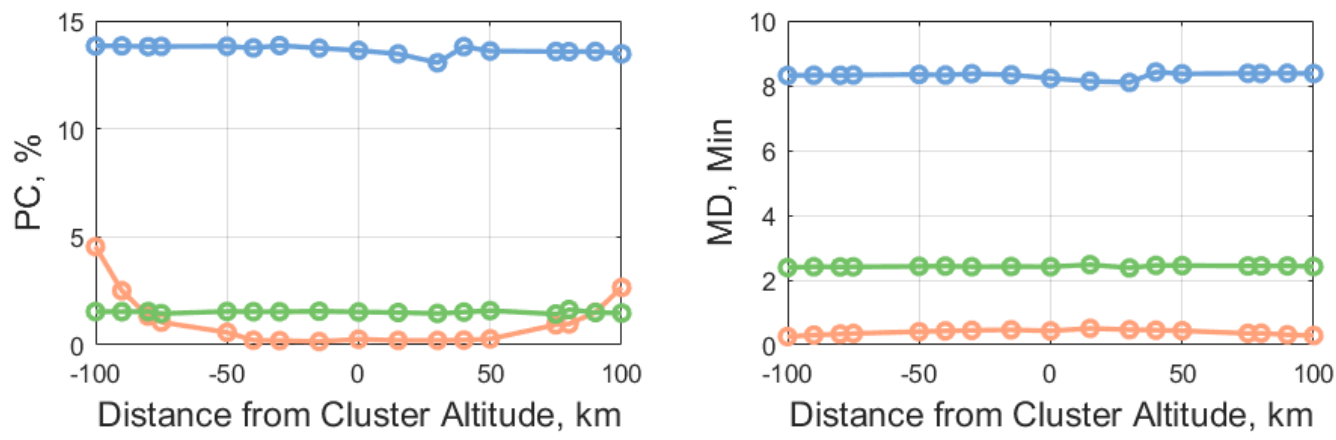

Fig. 17 Cluster 3 Circular Static Metrics 
Figures 15-17 indicate that the MRT and TAG metrics show the most variation, and therefore have the largest impact on total score. PC and MD are relatively steady across all cases, with the exception of Tier $1 \mathrm{PC}$, which begins to increase at the edge of the Tier 1 range constraint. The metric figures only display cases for offsets up to $\pm 100 \mathrm{~km}$ to highlight the region of interest. Offsets larger than 100 have no conjunctions in Tier 1, as seen in Tables 3-5, and negatively affect the scale of the figures. Tier $1 \mathrm{MRT}$ and TAG performance is best in the $\pm 50 \mathrm{~km}$ cases, which is driving the overall results. The results indicate that constellation performance is more dependent on the access focused metrics than the coverage focused metrics. With substantial relative movement between RSOs and OBSs, the access metrics are much more dependent on relative altitude.

Based solely on the circular constellation results, a constellation of observer satellites placed at the ideal altitude offset would have highly effective coverage of the debris cluster. On average, each RSO would have a Tier 3 level conjunction every hour with a long enough duration to engage in meaningful LDR, and a Tier 1 conjunction every day capable of close range SSA observation and characterization.

\section{B. Static Elliptical Cases}

Elliptical constellations increase the OBS to RSO relative movement within the debris clusters, and allow for more frequent conjunctions with the debris objects. Generally, elliptical cases do improve performance compared to the circular cases. However while the results show that on average the elliptical cases perform better, the top performing circular case for each cluster out performed every single elliptical case analyzed.

For Cluster 1, the average performance of all circular constellations was 2.13 with a standard deviation of 0.33 , while the elliptical constellations have an average performance of 2.29 with a 
standard deviation of 0.18 . The top performing circular case, $-50 \mathrm{~km}$ offset, has a score of 2.632 , while the top performing elliptical case, $-50 \mathrm{~km}$ apogee / $-100 \mathrm{~km}$ perigee offset, has a score of 2.545. There is only a small difference between these two cases, but the main distinction is in their Tier 1 performance, where the circular case outperforms the elliptical case in every metric. The elliptical constellations test set includes apogee/perigee combinations over the full range of circular cases analyzed. One area where elliptical constellations do perform better than circular cases though, is more frequent close conjunctions. The grading metrics are geared towards constellations that offer prolonged contact with the debris, but for a mission where achieving the closest possible approach is desired, the best performing case may be elliptical.

Figures 18-20 depict the total score of each case for each cluster, providing a summary of the performance for each constellation. Tables 7-9 show the full performance results for each cluster. The best performing circular case is included in the elliptical results tables as a reference for comparison.

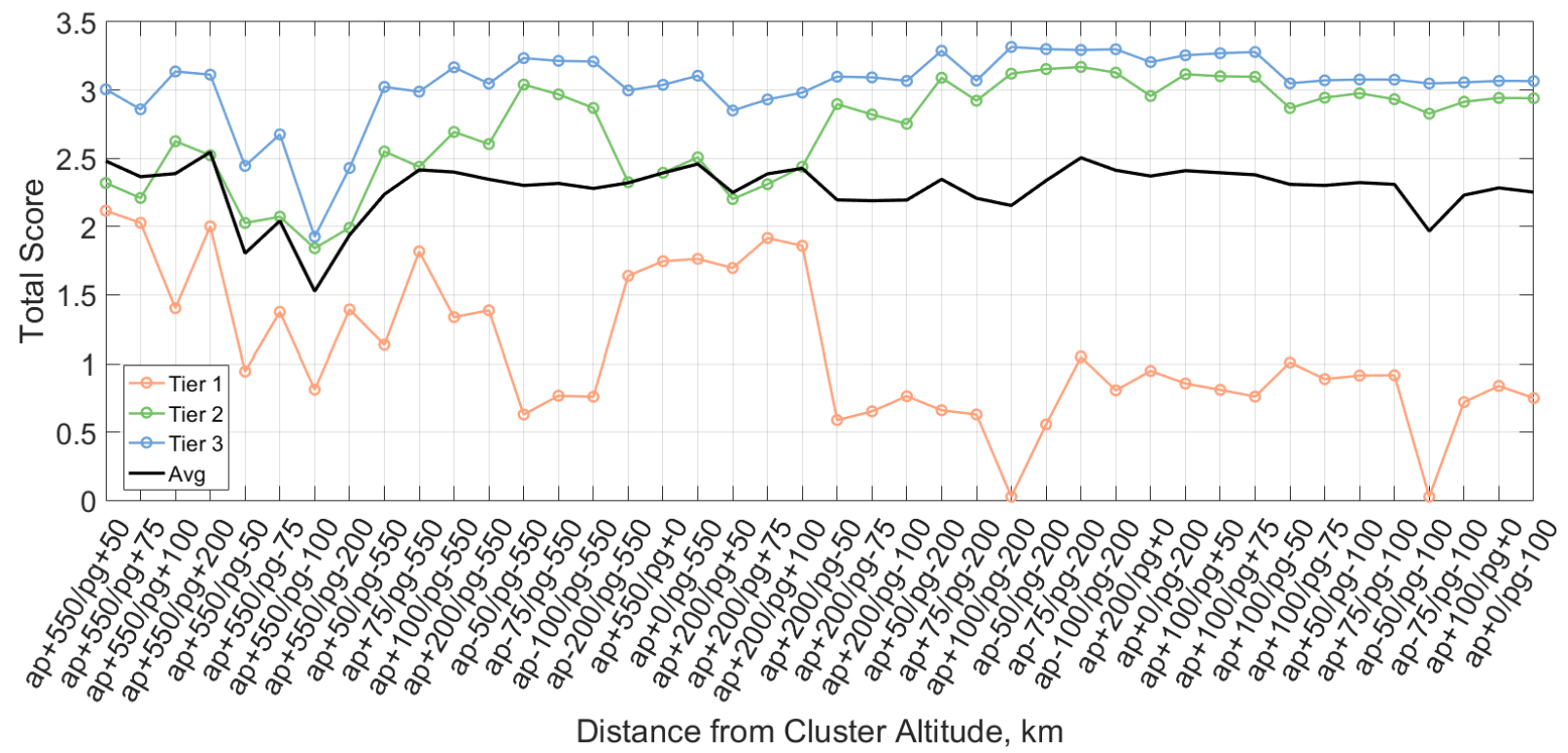

Fig. 18 Cluster 1 Elliptical Static Total Score 

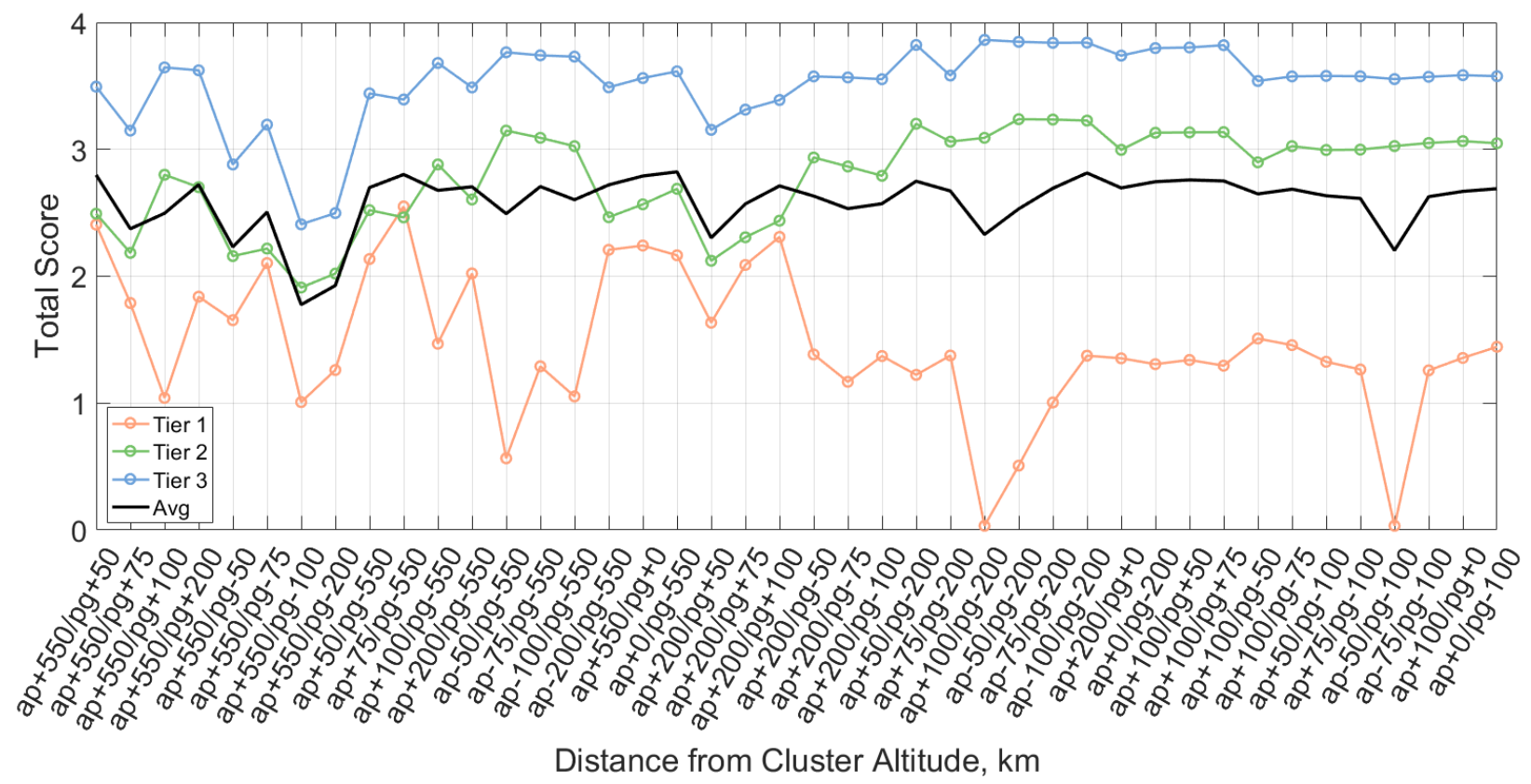

Fig. 19 Cluster 2 Elliptical Static Total Score

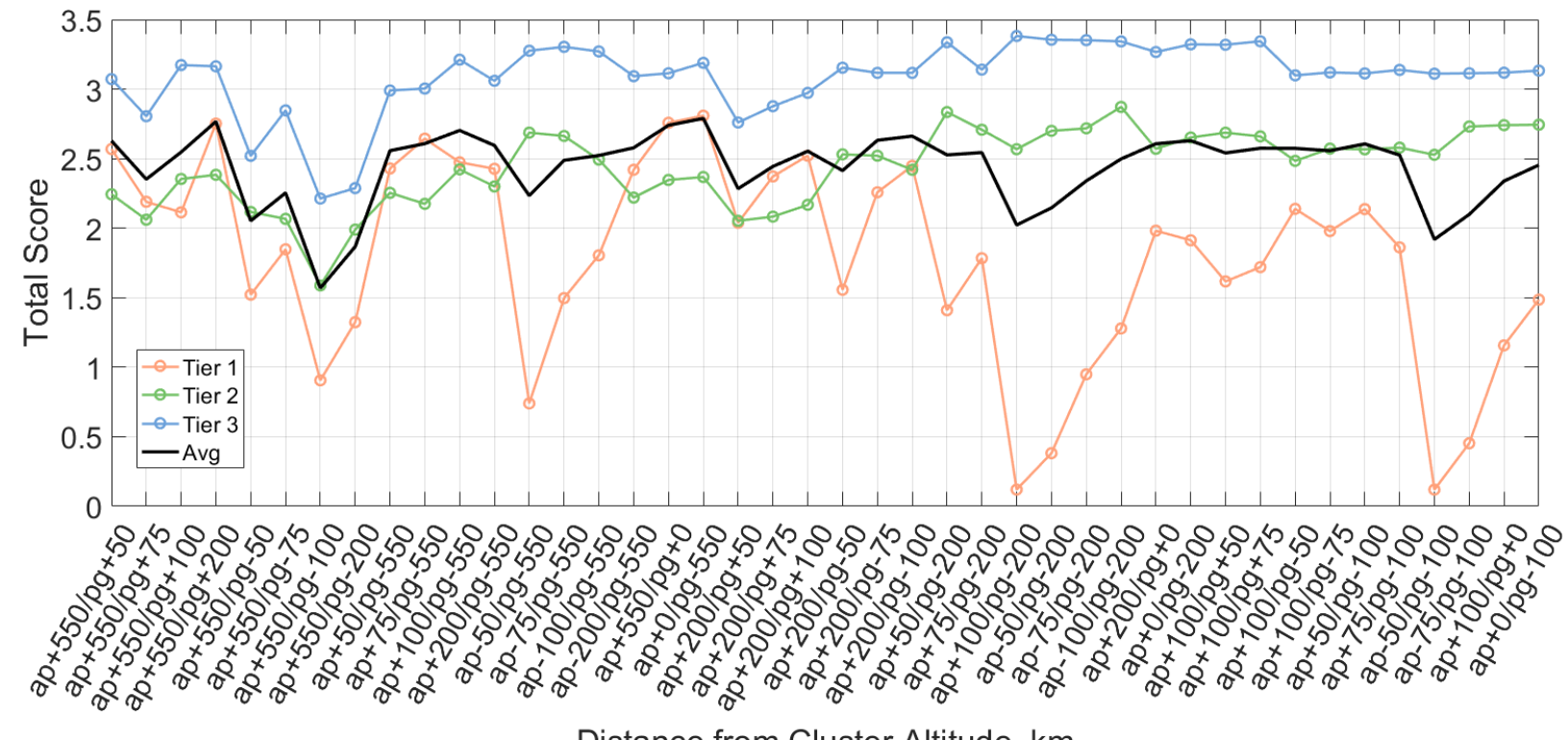

Distance from Cluster Altitude, $\mathrm{km}$

Fig. 20 Cluster 3 Elliptical Static Total Score 
Results for Clusters 2 and 3 closely resemble those for Cluster 1, with overall improved performance from elliptical cases, but the best performing case still being the $-50 \mathrm{~km}$ circular constellation. The best performing Cluster 2 and Cluster 3 elliptical constellation is the $50 \mathrm{~km}$ apogee / -200 km perigee case. The average performance of all Cluster 2 circular constellations is 2.33 with a standard deviation of 0.32 , while the elliptical constellations have an average performance score of 2.59 with a standard deviation of 0.22 . The average performance of all Cluster 3 circular constellations is 2.34 with a standard deviation of 0.35 , while the elliptical constellations have an average performance score of 2.44 with a standard deviation of 0.26 . The results are mostly consistent between all 3 clusters, and are compared in Table 6.

Table 6 Circular vs. Elliptical Result Statistics

\begin{tabular}{|c|c|c|c|c|}
\hline \multirow{2}{*}{ Cluster } & \multicolumn{2}{|c|}{ Circular } & \multicolumn{2}{c|}{ Elliptical } \\
\cline { 2 - 5 } & Total Score & 1 $\boldsymbol{\text { Std }}$ & Total Score & $\mathbf{1 \sigma}$ Std \\
\hline $\mathbf{1}$ & 2.13 & 0.33 & 2.29 & 0.18 \\
\hline $\mathbf{2}$ & 2.33 & 0.32 & 2.59 & 0.22 \\
\hline $\mathbf{3}$ & 2.34 & 0.35 & 2.44 & 0.26 \\
\hline
\end{tabular}

Given that the debris clusters themselves contain exclusively circular orbits, the circular designs more closely match the orbits of the debris objects. When positioned in an ideal offset from the cluster, circular cases offer more prolonged observation capabilities as a larger percentage of their time is spent within the conjunction distance of the debris objects. The metrics are geared towards finding the cases that best fit the mission parameters, and prolonged contact provides a better LDR operating environment. Comparatively though, elliptical constellations with large or poor performing altitude offsets still intersect the clusters and allow for conjunctions with the debris objects. Cases such as the elliptical $550 \mathrm{~km}$ apogee / -200 km perigee constellation perform better than either of its circular counterparts. 
Table 7 Cluster 1 Elliptical Static Performance Results

\begin{tabular}{|c|c|c|c|c|c|c|c|c|c|c|c|c|c|c|c|c|c|c|c|}
\hline & \multicolumn{6}{|c|}{ Tier 1: $100 \mathrm{~km}$} & \multicolumn{6}{|c|}{ Tier 2: $600 \mathrm{~km}$} & \multicolumn{6}{|c|}{ Tier 3: $2400 \mathrm{~km}$} & \multirow{2}{*}{$\begin{array}{l}\text { Total } \\
\text { Score }\end{array}$} \\
\hline Case & $\begin{array}{l}\text { MRT } \\
\text { (days) }\end{array}$ & $\begin{array}{c}\text { TAG } \\
\text { (days) }\end{array}$ & PC (\%) & $\begin{array}{c}\text { MeanD } \\
\text { (min) }\end{array}$ & $\mathrm{CC}(\%)$ & Score & $\begin{array}{l}\text { MRT } \\
\text { (days) }\end{array}$ & $\begin{array}{c}\text { TAG } \\
\text { (days) }\end{array}$ & PC (\%) & $\begin{array}{c}\text { MeanD } \\
(\min )\end{array}$ & $\mathrm{CC}(\%)$ & Score & $\begin{array}{l}\text { MRT } \\
\text { (days) }\end{array}$ & $\begin{array}{c}\text { TAG } \\
\text { (days) }\end{array}$ & PC (\%) & $\begin{array}{c}\text { MeanD } \\
(\mathrm{min})\end{array}$ & CC (\%) & Score & \\
\hline circ -50km 8obj 0Man & 0.938 & 1.573 & 0.905 & 0.534 & 100 & 2.556 & 0.454 & 0.857 & 1.865 & 2.849 & 100 & 2.333 & 0.026 & 0.050 & 14.408 & 8.669 & 100 & 3.006 & 2.632 \\
\hline ecc ap550 pg50 8obj 0Man & 16.09 & 22.04 & 8.58 & 0.28 & 100 & 0.750 & 0.171 & 0.301 & 1.373 & 2.537 & 100 & 2.940 & 0.024 & 0.047 & 13.463 & 8.629 & 100 & 3.066 & 2.252 \\
\hline ecc ap550 pg75 8obj 0Man & 23.18 & 30.07 & 15.64 & 0.12 & 100 & .839 & 0.165 & 0.299 & 1.352 & 2.485 & 100 & 2.942 & 0.024 & 0.047 & 13.366 & 8.627 & 100 & 3.067 & 2.283 \\
\hline ecc ap550 pg100 8obj 0Man & 62.35 & 64.45 & 13.04 & & 100 & 721 & 0.165 & 0.298 & 309 & 2.444 & 100 & & 0.025 & 0.047 & 13.366 & 8.631 & 100 & & 230 \\
\hline ecc ap550 pg200 8obj 0Man & 90.00 & 90.00 & 0.00 & 0.00 & 0 & 028 & 163 & 0.288 & 163 & 2.287 & 100 & 827 & 0.024 & 0.047 & 13.141 & 8.621 & 100 & .048 & 967 \\
\hline ecc ap550 pg-50 8obj 0Man & 5.952 & 9.119 & 6.187 & 0.347 & 100 & .917 & 0.174 & 0.316 & 432 & 2.588 & 100 & .933 & 0.024 & 0.047 & 13.673 & 8.623 & 100 & 3.076 & 309 \\
\hline ecc ap550 pg-75 8obj 0Man & 4.729 & 8.005 & 6.358 & 0.271 & 100 & 0.915 & 0.174 & 0.316 & 1.491 & 2.634 & 100 & 2.976 & 0.024 & 0.047 & 13.681 & 8.619 & 100 & 3.077 & .322 \\
\hline ecc ap550 pg-100 8obj 0Man & 5.512 & 8.458 & 5.793 & & 100 & .889 & .185 & 0.319 & 1.509 & & 100 & .944 & 0.024 & 0.047 & 13.724 & 8.619 & 100 & 3.071 & \\
\hline ecc ap $550 \mathrm{pg}-2$ & 3.907 & 6.292 & 3.310 & 398 & 100 & 010 & 196 & 0.360 & 1.568 & 2.696 & 10 & 8 & 0.025 & 0.047 & 13.831 & 611 & 100 & 3.049 & \\
\hline ecc ap50 pg-550 8obj 0Man & 7.263 & 10.517 & 5.258 & & 100 & & 155 & 0.291 & .505 & 529 & & 7 & 0.022 & 0.042 & 14.286 & 3.424 & & 279 & \\
\hline ecc ap75 pg-550 8obj 0Man & 5.539 & 9.147 & 4.628 & 0.291 & 100 & 0.811 & 0.156 & 0.294 & 1.529 & 2.546 & & & 0.022 & 0.042 & 14.305 & 8.432 & 100 & 3.270 & 394 \\
\hline ecc ap100 pg-550 8obj 0Man & 6.181 & 8.978 & 4.796 & & 100 & 0.857 & 0.156 & 0.298 & 1.566 & 2.577 & 100 & 3.116 & 0.022 & 0.042 & 14.292 & 8.439 & 100 & 3.255 & 2.409 \\
\hline ecc ap2 & 5.050 & 7.027 & 5.050 & & 100 & & 182 & 0.336 & 1.611 & 626 & 10 & & 0.023 & 0.043 & 14.289 & & 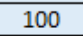 & & \\
\hline есc ap- $-50 \mathrm{pg}-5$ & 18.040 & 24.008 & 8.985 & & 100 & 0.807 & 146 & 0.274 & 434 & 2.435 & 10 & 8 & 0.021 & 0.041 & 4.197 & 396 & 100 & 299 & \\
\hline ecc ap- $75 \mathrm{pg}-55$ & .344 & .828 & 4.217 & 401 & 97.727 & .049 & .143 & 0.269 & 437 & 440 & & 99 & 0.021 & 0.041 & 4.146 & 387 & & .294 & 504 \\
\hline ecc ap-100 pg-550 8obj 0Man & 62.198 & 65.240 & 1.922 & 037 & 4.318 & 0.557 & 0.142 & 0.265 & 1.398 & 2.392 & 10 & .154 & 0.021 & 0.041 & 14.162 & 376 & & 3.300 & 337 \\
\hline ecc ap-2C & 90.000 & 90.000 & 0.000 & & & & & 0.255 & 1.264 & & & & 0.021 & 0.040 & & & & & \\
\hline ecc ap5 & 9.964 & 14.851 & 5.008 & & & 630 & 174 & 0.310 & 1.408 & 2.538 & & 2 & 0.025 & 0.047 & 13.594 & & & 69 & 207 \\
\hline ecc ap0 pg-550 8obj 0Man & .085 & .905 & 4.717 & & & 1 & 54 & 0.282 & 459 & & & & 0.022 & 0.041 & 1.254 & & & 88 & \\
\hline ecc ap200 pg50 8obj 0Man & 5.975 & 9.171 & 4.522 & 0.260 & 100 & 0.764 & 0.234 & 0.437 & 1.696 & 2.809 & 10 & 2.752 & 0.025 & 0.047 & 13.944 & 8.698 & 100 & 3.067 & 2.194 \\
\hline ecc ap200 pg75 8obj 0Man & 13.059 & 20.162 & 8.936 & & & 0.653 & 0.218 & 0.405 & 1.687 & & & & 0.024 & 0.047 & 13.943 & & & 3.093 & 2.189 \\
\hline ecc ap. & 55.241 & 57.742 & 12.051 & & & & & 0.378 & 1.700 & & & & 0.024 & 0.047 & & & & & \\
\hline ecc ap20 & 1.461 & 2.420 & 1.381 & & & 859 & 358 & 0.672 & 1.779 & 2.846 & & & 0.026 & 0.050 & 4.108 & 689 & 100 & 981 & 426 \\
\hline ecc ap200 pg-75 8obj 0Man & 469 & 2.479 & & & & & 425 & 0.795 & 1.750 & & & & 0.027 & 0.052 & 4.139 & & & 31 & 2.386 \\
\hline ecc ap200 pg-100 8obj 0Man & 1.895 & 3.081 & 1.386 & 654 & & 1.696 & 0.525 & 0.989 & 1.748 & 2.828 & 10 & 2.201 & 0.029 & 0.055 & 4.166 & 681 & 00 & .850 & 2.249 \\
\hline ecc ap: & 650 & 2.497 & & & & & & 0.6 & 1.7 & & & & 0.0 & 0.0 & & & & & \\
\hline ecc ap & 1.815 & 2.572 & 1 & & & 6 & 9 & 0.705 & 1.783 & & & & 0.026 & 0.048 & 397 & & & 38 & \\
\hline ecc ap100 pg-200 8obj 0Man & 1.915 & 3.224 & 1.135 & & & & & 0.852 & 1.862 & & & & 0.026 & 0.050 & 4.438 & 0 & 0 & 997 & 320 \\
\hline ecc ap-50 pg-200 8obj 0Man & 6.394 & 10.375 & 4.449 & 0.290 & & 0.760 & 0.212 & 0.397 & 1.747 & 2.778 & 100 & 2.869 & 0.023 & 0.044 & 14.317 & 8.597 & 100 & 3.209 & 2.279 \\
\hline ecc ap-75 pg-200 8obj 0Man & 18.399 & 18.044 & 9.855 & & & 0.767 & 0.196 & 0.368 & 1.773 & 2.764 & 100 & & 0.023 & 0.043 & 14.322 & 8.587 & 100 & 3.214 & 2.316 \\
\hline ecc ap-100 pg-20 & 55.832 & 55.588 & 11.320 & & 97.727 & & & 0.344 & & & & & 0.022 & 0.0 & & & & & \\
\hline ecc ap & 225 & 3.556 & 2.771 & & & & & 0.52 & 1.755 & 28 & & & 0.02 & 0.048 & 14.071 & 44 & & 47 & 2.346 \\
\hline есc ap0 pg-2 & 328 & 3.661 & 257 & & & & 33 & 0.475 & 1.758 & & & & 0.023 & 0.045 & 1.370 & 3 & 100 & .167 & 2.399 \\
\hline ecc ap100 pg50 8obj 0Man & 1.392 & 2.344 & 1.277 & 0.435 & & 1.818 & 0.361 & 0.672 & 1.780 & 2.854 & 100 & 2.438 & 0.026 & 0.050 & 14.102 & 8.705 & 100 & .988 & .415 \\
\hline ecc ap100 pg75 8obj 0Man & 2.590 & 4.264 & 2.541 & & & 1.134 & & 0.585 & 1.799 & 2.849 & & & 0.026 & 0.049 & 14.081 & 8.699 & & 3.023 & 236 \\
\hline ecc ap 100 & & 4.20 & & & & & & & & & & & 0.042 & 0.081 & & & & & \\
\hline ecc ap1 & 8.356 & 12.01 & & & & & & 7.10 & & & & & 0.0 & 0.1 & & & & 25 & 1.526 \\
\hline ecc ap50 pg-100 8obj 0Man & 3.271 & 3.427 & 0.606 & & 100 & 1.375 & 0.988 & 1.752 & 1.907 & 2.850 & 100 & & 0.035 & 0.062 & 14.596 & 8.705 & 100 & 2.675 & 2.041 \\
\hline ecc ap75 pg-100 8obj 0Man & 16.853 & 6.867 & 0.557 & 0.655 & 100 & 0.944 & 3.528 & 3.331 & 2.016 & 3.016 & 100 & 2.026 & 0.058 & 0.067 & 15.122 & 8.878 & 100 & 2.444 & 1.804 \\
\hline ecc ap-50 pg-100 8obj 0Man & 1.265 & 2.072 & 1.218 & & & 2.001 & 0.317 & 0.601 & 1.790 & 2.829 & 10 & 2 & 0.024 & 0.047 & 14.350 & 8.636 & 100 & 3.113 & 2.545 \\
\hline ecc ap-75 pg-100 8obj 0Man & 2.040 & 3.159 & 1.960 & & & & & 0.527 & 1.8 & & & & 0.024 & 0.046 & & & 100 & .136 & 2.388 \\
\hline & 1.289 & 2.243 & & & & & & 1.000 & 1.72 & & & & 0.029 & 0.055 & & & 100 & .859 & 2.365 \\
\hline ecc ap0 pg-100 8obj 0Man & 1.218 & 2.081 & 1.096 & 0.562 & 100 & 2.454 & 0.460 & 0.858 & 1.843 & 2.848 & 100 & 2.319 & 0.026 & 0.050 & 14.447 & 8.672 & 100 & 3.005 & 2.593 \\
\hline
\end{tabular}


Table 8 Cluster 2 Elliptical Static Performance Results

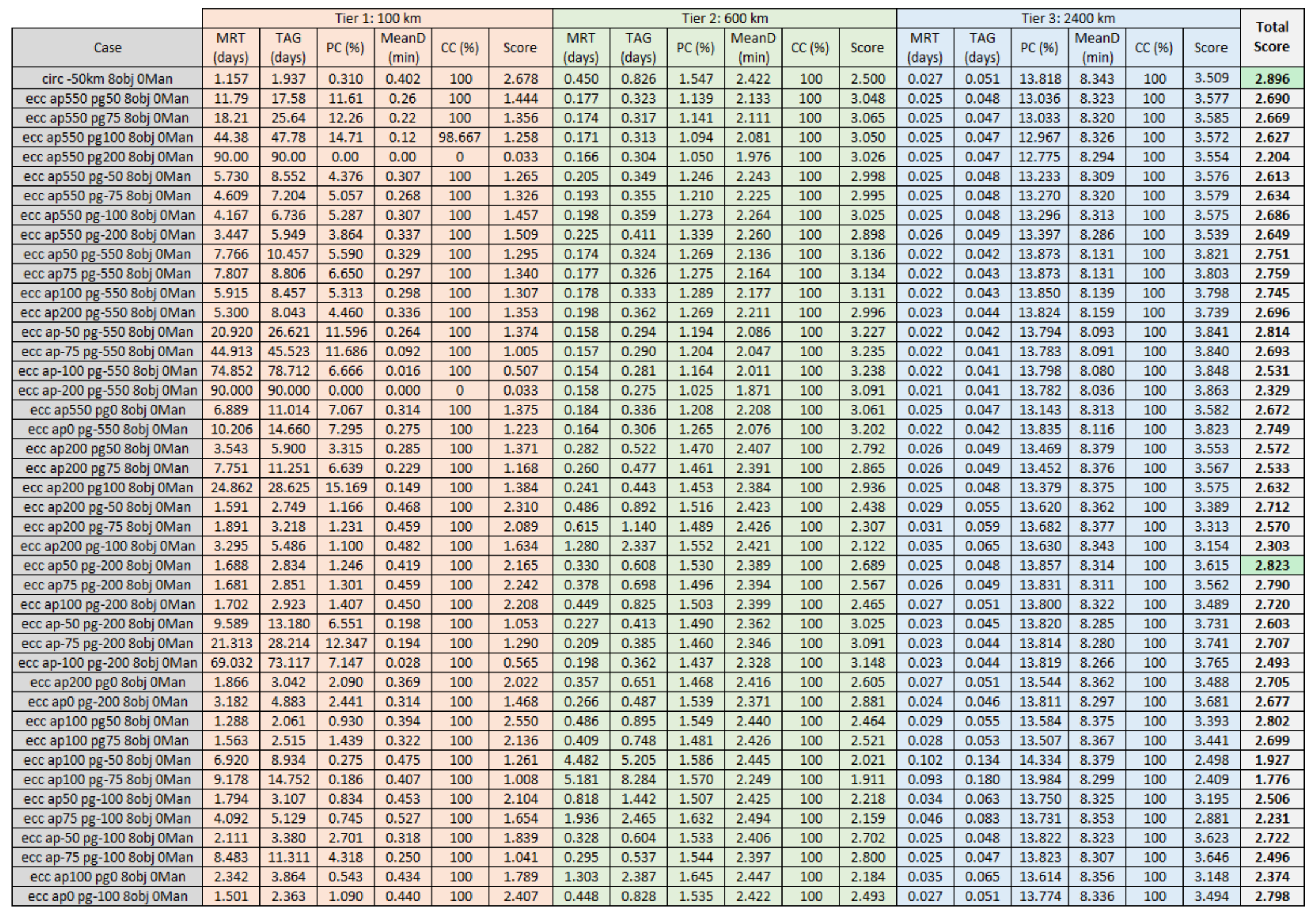




\section{Table 9 Cluster 3 Elliptical Static Performance Results}

\begin{tabular}{|c|c|c|c|c|c|c|c|c|c|c|c|c|c|c|c|c|c|c|c|}
\hline \multirow{3}{*}{ Case } & & & & & & & & & & & & & & & & & & & \\
\hline & \multicolumn{6}{|c|}{ Tier 1: $100 \mathrm{~km}$} & \multicolumn{6}{|c|}{ Tier 2: $600 \mathrm{~km}$} & \multicolumn{6}{|c|}{ Tier $3: 2400 \mathrm{~km}$} & \multirow{2}{*}{$\begin{array}{l}\text { Total } \\
\text { Score }\end{array}$} \\
\hline & $\begin{array}{l}\text { MRT } \\
\text { (days) }\end{array}$ & $\begin{array}{c}\text { TAG } \\
\text { (days) }\end{array}$ & PC (\%) & $\begin{array}{c}\text { MeanD } \\
\text { (min) }\end{array}$ & $\mathrm{CC}(\%)$ & Score & $\begin{array}{l}\text { MRT } \\
\text { (days) }\end{array}$ & $\begin{array}{c}\text { TAG } \\
\text { (days) }\end{array}$ & PC (\%) & \begin{tabular}{|c} 
Meand \\
$(\mathrm{min})$
\end{tabular} & $\mathrm{CC}(\%)$ & Score & $\begin{array}{l}\text { MRT } \\
\text { (days) }\end{array}$ & $\begin{array}{c}\text { TAG } \\
\text { (days) }\end{array}$ & PC (\%) & \begin{tabular}{|c|} 
Meand \\
$(\mathrm{min})$
\end{tabular} & $\mathrm{CC}(\%)$ & Score & \\
\hline circ -50km 8obj 0Man & 1.469 & 2.314 & 0.380 & 0.399 & 100 & 2.697 & 0.450 & 0.826 & 1.547 & 2.422 & 100 & 2.308 & 0.027 & 0.051 & 13.818 & 8.343 & 100 & 3.537 & 2.847 \\
\hline ecc ap550 pg50 8obj 0Man & 17.13 & 18.09 & 11.38 & 0.36 & 100 & 1.486 & 0.237 & 0.407 & 1.521 & 2.128 & 100 & 2.744 & 0.031 & 0.055 & 12.348 & 7.159 & 100 & 3.133 & 2.454 \\
\hline ecc ap550 pg75 8obj 0Man & 20.70 & 22.46 & 8.63 & 0.28 & 100 & 1.157 & 0.232 & 0.397 & 1.477 & 2.107 & 100 & 2.741 & 0.030 & 0.052 & 11.808 & 6.856 & 100 & 3.118 & 2.339 \\
\hline ecc ap550 pg100 8obj 0Man & 25.96 & 27.09 & 4.01 & 0.05 & 99.667 & 0.453 & 0.229 & 0.389 & 1.441 & 2.076 & 100 & 2.731 & 0.030 & 0.052 & 11.721 & 6.845 & 100 & 3.114 & 2.099 \\
\hline ecc ap550 pg200 8obj 0Man & 31.00 & 31.00 & 0.00 & 0.00 & 0 & 0.120 & 0.233 & 0.396 & 1.249 & 1.854 & 100 & 2.528 & 0.032 & 0.055 & 12.124 & 7.159 & 100 & 3.111 & 1.919 \\
\hline ecc ap550 pg-50 8obj 0Man & 6.569 & 8.810 & 11.351 & 0.421 & 100 & 1.862 & 0.300 & 0.473 & 1.543 & 2.191 & 100 & 2.579 & 0.032 & 0.057 & 12.869 & 7.349 & 100 & 3.138 & 2.526 \\
\hline ecc ap550 pg-75 8obj 0Man & 5.971 & 7.639 & 11.661 & 0.532 & 100 & 2.137 & 0.282 & 0.480 & 1.495 & 2.169 & 100 & 2.567 & 0.032 & 0.056 & 12.540 & 7.162 & 100 & 3.113 & 2.606 \\
\hline ecc ap550 pg-100 8obj 0Man & 5.647 & 7.278 & 13.099 & 0.381 & 100 & 1.979 & 0.275 & 0.462 & 1.496 & 2.100 & 100 & 2.572 & 0.030 & 0.053 & 12.114 & 6.888 & 100 & 3.120 & 2.557 \\
\hline ecc ap550 pg-200 8obj 0Man & 3.812 & 5.011 & 11.214 & 0.382 & 100 & 2.139 & 0.345 & 0.597 & 1.660 & 2.221 & 100 & 2.484 & 0.033 & 0.057 & 12.762 & 7.179 & 100 & 3.099 & 2.574 \\
\hline ecc ap50 pg-550 8obj 0Man & 5.138 & 7.095 & 10.851 & 0.287 & 100 & 1.720 & 0.250 & 0.431 & 1.543 & 2.050 & 100 & 2.660 & 0.028 & 0.048 & 13.085 & 6.975 & 100 & 3.344 & 2.575 \\
\hline ecc ap75 pg-550 8obj 0Man & 4.683 & 6.672 & 9.116 & 0.258 & 100 & 1.617 & 0.266 & 0.454 & 1.622 & 2.172 & 100 & 2.687 & 0.026 & 0.047 & 12.485 & 6.655 & 100 & 3.319 & 2.541 \\
\hline ecc ap100 pg-550 8obj 0Man & 4.214 & 6.012 & 10.538 & 0.339 & 100 & 1.913 & 0.252 & 0.436 & 1.543 & 2.056 & 100 & 2.652 & 0.027 & 0.049 & 12.890 & 6.889 & 100 & 3.322 & 2.629 \\
\hline ecc ap200 pg-550 8obj 0Man & 5.134 & 6.399 & 12.973 & 0.348 & 100 & 1.982 & 0.287 & 0.486 & 1.557 & 2.140 & 100 & 2.571 & 0.028 & 0.049 & 12.637 & 6.784 & 100 & 3.267 & 2.607 \\
\hline ecc ap-50 pg-550 8obj 0Man & 17.556 & 17.913 & 10.049 & 0.286 & 100 & 1.279 & 0.198 & 0.342 & 1.465 & 1.959 & 100 & 2.872 & 0.029 & 0.050 & 13.435 & 7.169 & 100 & 3.343 & 2.498 \\
\hline ecc ap-75 pg-550 8obj 0Man & 22.026 & 22.873 & 9.345 & 0.147 & 100 & 0.949 & 0.231 & 0.396 & 1.501 & 2.014 & 100 & 2.718 & 0.027 & 0.047 & 12.794 & 6.831 & 100 & 3.352 & 2.340 \\
\hline ecc ap-100 pg-550 8obj 0Man & 27.099 & 27.880 & 3.707 & 0.022 & 99.333 & 0.382 & 0.216 & 0.365 & 1.381 & 1.883 & 100 & 2.700 & 0.028 & 0.048 & 13.092 & 6.973 & 100 & 3.355 & 2.146 \\
\hline ecc ap-200 pg-550 8obj 0Man & 31.000 & 31.000 & 0.000 & 0.000 & 0 & 0.120 & 0.227 & 0.366 & 1.248 & 1.797 & 100 & 2.568 & 0.026 & 0.046 & 12.603 & 6.684 & 100 & 3.382 & 2.023 \\
\hline ecc ap550 pg0 8obj 0Man & 9.631 & 12.323 & 12.506 & 0.422 & 100 & 1.784 & 0.250 & 0.430 & 1.559 & 2.145 & 100 & 2.708 & 0.032 & 0.055 & 12.561 & 7.226 & 100 & 3.140 & 2.544 \\
\hline ecc ap0 pg-550 8obj 0Man & 9.435 & 11.315 & 11.792 & 0.222 & 100 & 1.410 & 0.210 & 0.362 & 1.519 & 1.994 & 100 & 2.835 & 0.027 & 0.047 & 12.779 & 6.818 & 100 & 3.337 & 2.527 \\
\hline ecc ap200 pg50 8obj 0Man & 4.047 & 5.494 & 12.070 & 0.564 & 100 & 2.448 & 0.436 & 0.759 & 1.799 & 2.311 & 100 & 2.420 & 0.033 & 0.058 & 12.939 & 7.321 & 100 & 3.117 & 2.662 \\
\hline ecc ap200 pg75 8obj 0Man & 8.455 & 10.209 & 17.822 & 0.488 & 100 & 2.258 & 0.376 & 0.638 & 1.786 & 2.306 & 100 & 2.521 & 0.033 & 0.058 & 12.968 & 7.344 & 100 & 3.117 & 2.632 \\
\hline ecc ap200 pg100 8obj 0Man & 17.896 & 19.567 & 13.355 & 0.344 & 99.667 & 1.557 & 0.334 & 0.569 & 1.704 & 2.205 & 100 & 2.531 & 0.032 & 0.057 & 12.972 & 7.385 & 100 & 3.154 & 2.414 \\
\hline ecc ap200 pg-50 8obj 0Man & 1.860 & 2.846 & 2.318 & 0.460 & 100 & 2.521 & 0.675 & 1.146 & 1.779 & 2.244 & 100 & 2.168 & 0.036 & 0.063 & 12.822 & 7.160 & 100 & 2.973 & 2.554 \\
\hline ecc ap200 pg-75 8obj 0Man & 2.030 & 3.291 & 1.790 & 0.489 & 100 & 2.372 & 0.863 & 1.482 & 1.767 & 2.272 & 100 & 2.083 & 0.037 & 0.065 & 12.406 & 6.908 & 100 & 2.877 & 2.444 \\
\hline ecc ap200 pg-100 8obj 0Man & 2.706 & 4.293 & 1.561 & 0.500 & 100 & 2.039 & 1.904 & 3.219 & 1.952 & 2.403 & 100 & 2.053 & 0.043 & 0.073 & 12.546 & 6.984 & 100 & 2.760 & 2.284 \\
\hline ecc ap50 pg-200 8obj 0Man & 1.530 & 2.292 & 3.037 & 0.396 & 100 & 2.809 & 0.463 & 0.790 & 1.815 & 2.237 & 100 & 2.368 & 0.033 & 0.058 & 13.652 & 7.450 & 100 & 3.189 & 2.789 \\
\hline ecc ap75 pg-200 8obj 0Man & 1.520 & 2.414 & 2.659 & 0.404 & 100 & 2.758 & 0.561 & 0.959 & 1.877 & 2.370 & 100 & 2.347 & 0.032 & 0.055 & 12.631 & 6.903 & 100 & 3.114 & 2.740 \\
\hline ecc ap100 pg-200 8obj 0Man & 1.945 & 3.051 & 2.175 & 0.456 & 100 & 2.420 & 0.788 & 1.336 & 1.971 & 2.286 & 100 & 2.220 & 0.035 & 0.061 & 13.482 & 7.394 & 100 & 3.093 & 2.578 \\
\hline ecc ap-50 pg-200 8obj 0Man & 5.192 & 6.701 & 12.897 & 0.262 & 100 & 1.805 & 0.369 & 0.628 & 1.739 & 2.261 & 100 & 2.493 & 0.029 & 0.052 & 13.125 & 7.156 & 100 & 3.271 & 2.523 \\
\hline ecc ap-75 pg-200 8obj 0Man & 12.135 & 14.090 & 14.853 & 0.216 & 100 & 1.497 & 0.296 & 0.505 & 1.749 & 2.218 & 100 & 2.663 & 0.030 & 0.053 & 13.718 & 7.478 & 100 & 3.304 & 2.488 \\
\hline ecc ap-100 pg-200 8obj 0Man & 23.192 & 24.019 & 9.166 & 0.039 & 99.333 & 0.740 & 0.276 & 0.468 & 1.695 & 2.167 & 100 & 2.687 & 0.028 & 0.049 & 12.523 & 6.813 & 100 & 3.276 & 2.234 \\
\hline ecc ap200 pg0 8obj 0Man & 2.015 & 3.085 & 4.109 & 0.418 & 100 & 2.427 & 0.501 & 0.845 & 1.741 & 2.261 & 100 & 2.300 & 0.034 & 0.059 & 12.741 & 7.154 & 100 & 3.060 & 2.595 \\
\hline ecc ap0 pg-200 8obj 0Man & 1.862 & 2.739 & 5.506 & 0.315 & 100 & 2.474 & 0.434 & 0.755 & 1.824 & 2.280 & 100 & 2.423 & 0.029 & 0.051 & 12.553 & 6.859 & 100 & 3.212 & 2.703 \\
\hline ecc ap100 pg50 8obj 0Man & 1.594 & 2.429 & 2.230 & 0.381 & 100 & 2.644 & 0.725 & 1.247 & 1.773 & 2.344 & 100 & 2.175 & 0.038 & 0.067 & 13.446 & 7.539 & 100 & 3.004 & 2.608 \\
\hline ecc ap100 pg75 8obj 0Man & 1.832 & 2.632 & 3.403 & 0.330 & 100 & 2.429 & 0.580 & 0.981 & 1.775 & 2.294 & 100 & 2.254 & 0.034 & 0.058 & 12.146 & 6.843 & 100 & 2.990 & 2.557 \\
\hline ecc ap100 pg-50 8obj 0Man & 8.365 & 8.423 & 1.184 & 0.460 & 100 & 1.324 & 6.660 & 7.162 & 1.993 & 2.424 & 100 & 1.990 & 0.132 & 0.159 & 13.934 & 7.408 & 100 & 2.288 & 1.867 \\
\hline ecc ap100 pg-75 8obj 0Man & 16.583 & 15.438 & 0.903 & 0.351 & 100 & 0.906 & 8.293 & 10.125 & 1.653 & 1.868 & 100 & 1.588 & 0.121 & 0.215 & 13.650 & 7.368 & 100 & 2.213 & 1.569 \\
\hline ecc ap50 pg-100 8obj 0Man & 2.978 & 4.111 & 0.674 & 0.436 & 100 & 1.848 & 1.182 & 1.930 & 1.842 & 2.339 & 100 & 2.067 & 0.044 & 0.076 & 13.489 & 7.428 & 100 & 2.847 & 2.254 \\
\hline ecc ap75 pg-100 8obj 0Man & 4.238 & 6.125 & 0.683 & 0.435 & 100 & 1.522 & 2.901 & 3.420 & 2.068 & 2.493 & 100 & 2.117 & 0.056 & 0.092 & 12.451 & 6.889 & 100 & 2.520 & 2.053 \\
\hline ecc ap-50 pg-100 8obj 0Man & 1.547 & 2.239 & 3.205 & 0.351 & 100 & 2.752 & 0.462 & 0.787 & 1.825 & 2.260 & 100 & 2.384 & 0.031 & 0.055 & 12.946 & 7.090 & 100 & 3.164 & 2.767 \\
\hline ecc ap-75 pg-100 8obj 0Man & 2.609 & 3.255 & 5.748 & 0.305 & 100 & 2.114 & 0.481 & 0.838 & 1.790 & 2.304 & 100 & 2.354 & 0.031 & 0.053 & 12.764 & 6.976 & 100 & 3.173 & 2.547 \\
\hline ecc ap100 pg0 8obj 0Man & 2.196 & 3.374 & 1.064 & 0.450 & 100 & 2.190 & 1.117 & 1.968 & 1.804 & 2.361 & 100 & 2.061 & 0.045 & 0.077 & 13.267 & 7.406 & 100 & 2.805 & 2.352 \\
\hline ecc ap0 pg-100 8obj 0Man & 1.607 & 2.481 & 1.088 & 0.390 & 100 & 2.569 & 0.637 & 1.090 & 1.845 & 2.297 & 100 & 2.244 & 0.034 & 0.059 & 12.942 & 7.124 & 100 & 3.072 & 2.628 \\
\hline
\end{tabular}




\section{Phasing Maneuver Cases}

Based on the similarity between all three clusters in examining the non-maneuvering circular and elliptical cases, the impact of maneuvering cases is only analyzed for Cluster 1 . The impact that maneuvering has on Cluster 1 performance can be assumed consistent with the other 2 clusters. For the phasing maneuver, each OBS propagates its orbit for 10 days before analyzing its performance over those 10 days. If the performance lowers the overall average, the satellite will maneuver and phase 10 degrees along its orbit. If the 10 days of propagation improve its performance, it will continue to orbit for another 10 days and then reevaluate.

The maneuvering cases were hypothesized to allow the OBSs to settle on the optimal position within their orbit, thereby improving the performance for every case. The results show that maneuvering cases have mixed results, but overall perform worse than non-maneuvering cases for circular constellations and perform slightly better for elliptical constellations. There is not a drastic difference in their performance, but maneuvering cases spend a large portion of time on their phasing orbits, and tend to not conjunct with any debris objects while phasing. Even though OBS performance is mostly improved after maneuvers, improvement is only slight and their time spent phasing increases their average response time. The TAG and MRT metrics decreased, but PC and MD metrics increased across every Tier. Despite time spent phasing reducing the overall conjunction time, the phasing maneuvers increase the PC metric enough for a net gain. Figures 21-22 show the total score for each Tier. Tables 10-11 contain the full performance results for circular and elliptical cases. 


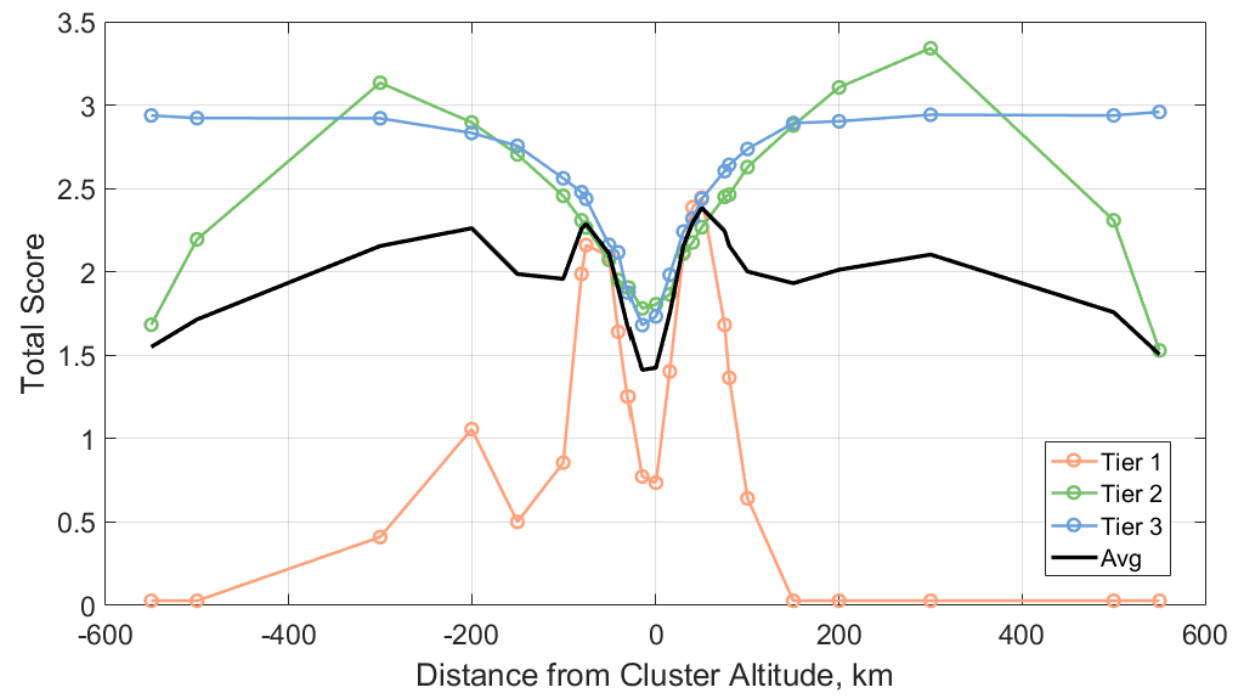

Fig. 21 Cluster 1 Circular Phasing Maneuver Total Score

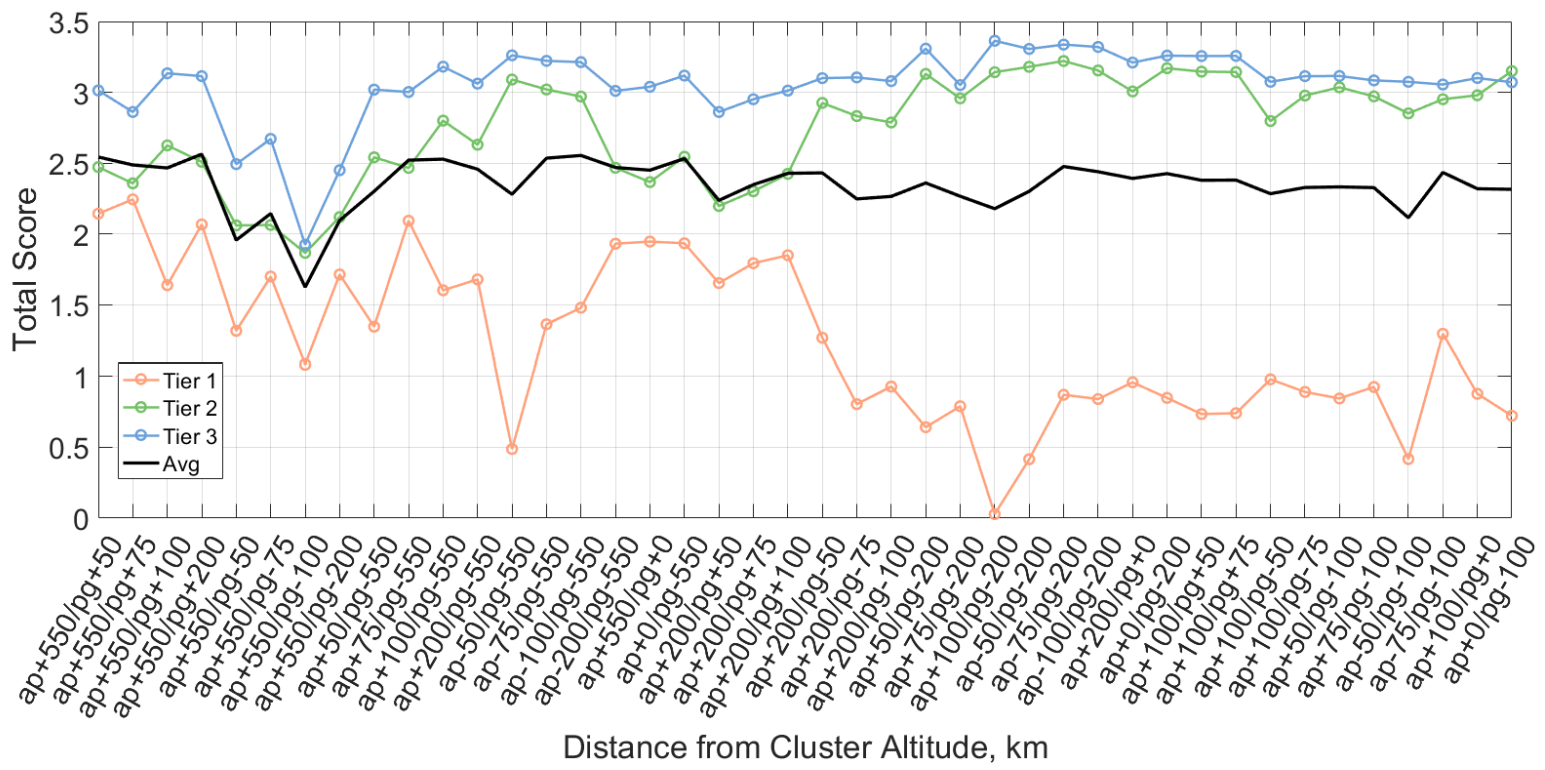

Fig. 22 Cluster 1 Elliptical Phasing Maneuver Total Score

It holds true for the phasing maneuver cases that elliptical constellations as a whole have better performance results than circular cases, but the best overall constellation remains the static, circular $-50 \mathrm{~km}$ offset case. Comparing the static and maneuvering cases for circular constellation designs, the static cases have an average score of 2.13 with a standard deviation of 0.33 , while the 
maneuvering cases have an average score of 1.99 with a standard deviation of 0.31 . The largest impact on the metrics was a doubling of the average Tier 3 TAG metric from 1.37 hours to 2.58 hours. The improvement in the PC metric is only slight, with a $4.8 \%$ increase in Tier 3 being the largest improvement.

Comparing the static and maneuvering cases for elliptical constellation designs, the static cases have an average score of 2.28 with a standard deviation of 0.19 , while the maneuvering cases have an average score of 2.35 with a standard deviation of 0.17 . Improvement is only slight, and with a $\Delta V$ of roughly $140 \mathrm{~m} / \mathrm{s}$ per maneuver, a steep improvement in at least one of the metrics is necessary for this maneuver to be considered a viable consideration in the constellation design. By any measure, adding a phasing maneuver to the constellation architecture does not provide an improvement in constellation performance.

Table 10 Cluster 1 Circular Phasing Maneuver Performance Results

\begin{tabular}{|c|c|c|c|c|c|c|c|c|c|c|c|c|c|c|c|c|c|c|c|}
\hline \multirow[b]{2}{*}{ Case } & \multicolumn{6}{|c|}{ Tier 1: $100 \mathrm{~km}$} & \multicolumn{6}{|c|}{ Tier 2: $600 \mathrm{~km}$} & \multicolumn{6}{|c|}{ Tier $3: 2400 \mathrm{~km}$} & \multirow{2}{*}{$\begin{array}{l}\text { Total } \\
\text { Score }\end{array}$} \\
\hline & $\begin{array}{c}\text { MRT } \\
\text { (days) }\end{array}$ & $\begin{array}{c}\text { TAG } \\
\text { (days) }\end{array}$ & PC (\%) & \begin{tabular}{|c} 
MeanD \\
$(\mathrm{min})$
\end{tabular} & CC (\%) & Score & $\begin{array}{c}\text { MRT } \\
\text { (days) }\end{array}$ & $\begin{array}{c}\text { TAG } \\
\text { (days) }\end{array}$ & PC (\%) & \begin{tabular}{|c} 
MeanD \\
$(\mathrm{min})$
\end{tabular} & CC (\%) & Score & $\begin{array}{c}\text { MRT } \\
\text { (days) }\end{array}$ & $\begin{array}{c}\text { TAG } \\
\text { (days) }\end{array}$ & PC (\%) & $\begin{array}{c}\text { MeanD } \\
(\mathrm{min}) \\
\end{array}$ & CC (\%) & Score & \\
\hline circ -50km 8obj 0Man & 0.938 & 1.573 & 0.905 & 0.534 & 100 & 2.556 & 0.454 & 0.857 & 1.865 & 2.849 & 100 & 2.333 & 0.026 & 0.050 & 14.408 & 8.669 & 100 & 3.006 & 2.632 \\
\hline circ $+550 \mathrm{~km} 8$ obj $1 \mathrm{Man}$ & 90.00 & 90.00 & 0.00 & 0.00 & 0 & 0.028 & 0.263 & 0.483 & 0.675 & 1.323 & 100 & 1.683 & 0.026 & 0.051 & 13.456 & 8.522 & 100 & 2.938 & 1.550 \\
\hline circ $+500 \mathrm{~km}$ 8obj $1 \mathrm{Man}$ & 90.00 & 90.00 & 0.00 & 0.00 & 0 & 0.028 & 0.197 & 0.356 & 0.804 & 1.717 & 100 & 2.195 & 0.027 & 0.051 & 13.741 & 8.525 & 100 & 2.922 & 1.715 \\
\hline circ $+300 \mathrm{~km}$ 8obj $1 \mathrm{Man}$ & 79.03 & 78.71 & 8.73 & 0.03 & 98.864 & 0.410 & 0.147 & 0.275 & 1.400 & 2.524 & 100 & 3.134 & 0.027 & 0.053 & 14.368 & 8.652 & 100 & 2.921 & 2.155 \\
\hline circ $+200 \mathrm{~km}$ 8obj $1 \mathrm{Man}$ & 61.23 & 65.77 & 17.85 & 0.31 & 96.591 & 1.057 & 0.193 & 0.357 & 1.589 & 2.711 & 100 & 2.896 & 0.030 & 0.057 & 14.693 & 8.688 & 100 & 2.833 & 2.262 \\
\hline circ $+150 \mathrm{~km}$ 8obj $1 \mathrm{Man}$ & 66.314 & 72.021 & 6.804 & 0.195 & 96.591 & 0.501 & 0.242 & 0.443 & 1.676 & 2.768 & 100 & 2.704 & 0.031 & 0.061 & 14.671 & 8.689 & 100 & 2.756 & 1.987 \\
\hline circ $+100 \mathrm{~km}$ 8obj $1 \mathrm{Man}$ & 5.413 & 8.095 & 4.206 & 0.328 & 100 & 0.856 & 0.336 & 0.631 & 1.747 & 2.812 & 100 & 2.456 & 0.038 & 0.073 & 15.106 & 8.685 & 100 & 2.561 & 1.958 \\
\hline circ $+80 \mathrm{~km}$ 8obj $1 \mathrm{Man}$ & 1.221 & 2.071 & 1.382 & 0.417 & 100 & 1.988 & 0.427 & 0.797 & 1.747 & 2.826 & 100 & 2.309 & 0.041 & 0.079 & 14.954 & 8.695 & 100 & 2.478 & 2.258 \\
\hline circ $+75 \mathrm{~km}$ 8obj $1 \mathrm{Man}$ & 1.115 & 1.920 & 1.257 & 0.464 & 100 & 2.161 & 0.455 & 0.847 & 1.724 & 2.833 & 100 & 2.266 & 0.043 & 0.082 & 14.817 & 8.698 & 100 & 2.438 & 2.288 \\
\hline circ $+50 \mathrm{~km} 8 \mathrm{obj} 1 \mathrm{Man}$ & 1.165 & 2.140 & 0.639 & 0.543 & 100 & 2.094 & 0.704 & 1.336 & 1.739 & 2.811 & 100 & 2.072 & 0.062 & 0.119 & 15.160 & 8.686 & 100 & 2.162 & 2.110 \\
\hline circ $+40 \mathrm{~km}$ 8obj $1 \mathrm{Man}$ & 1.636 & 2.993 & 0.330 & 0.545 & 100 & 1.641 & 1.021 & 1.883 & 1.702 & 2.808 & 100 & 1.951 & 0.066 & 0.127 & 15.204 & 8.645 & 100 & 2.117 & 1.903 \\
\hline circ +30km 8obj 1Man & 2.908 & 4.943 & 0.191 & 0.623 & 100 & 1.253 & 1.731 & 3.124 & 1.757 & 2.893 & 100 & 1.907 & 0.112 & 0.187 & 14.256 & 8.768 & 100 & 1.876 & 1.679 \\
\hline circ $+15 \mathrm{~km}$ 8obj 1 Man & 10.399 & 15.574 & 0.135 & 0.594 & 100 & 0.773 & 4.881 & 7.687 & 1.807 & 2.710 & 100 & 1.781 & 0.250 & 0.468 & 15.141 & 8.658 & 100 & 1.681 & 1.412 \\
\hline circ +0km 8obj 1Man & 12.654 & 10.484 & 0.258 & 0.516 & 100 & 0.736 & 3.821 & 5.702 & 1.736 & 2.830 & 100 & 1.806 & 0.203 & 0.383 & 15.182 & 8.796 & 100 & 1.733 & 1.425 \\
\hline circ-15km 8obj 1Man & 2.379 & 3.895 & 0.698 & 0.594 & 100 & 1.403 & 2.174 & 2.378 & 1.718 & 2.794 & 100 & 1.865 & 0.083 & 0.150 & 14.542 & 8.623 & 100 & 1.982 & 1.750 \\
\hline circ-30km 8obj 1Man & 1.166 & 2.121 & 0.611 & 0.566 & 100 & 2.120 & 0.691 & 1.245 & 1.794 & 2.798 & 100 & 2.109 & 0.057 & 0.109 & 15.726 & 8.681 & 100 & 2.242 & 2.157 \\
\hline circ $-40 \mathrm{~km}$ 8obj 1Man & 1.028 & 1.725 & 0.683 & 0.552 & 100 & 2.387 & 0.585 & 1.041 & 1.791 & 2.800 & 100 & 2.176 & 0.049 & 0.094 & 15.117 & 8.630 & 100 & 2.320 & 2.294 \\
\hline circ -50km 8obj 1Man & 0.960 & 1.643 & 0.881 & 0.490 & 100 & 2.445 & 0.461 & 0.866 & 1.756 & 2.823 & 100 & 2.268 & 0.043 & 0.083 & 15.233 & 8.645 & 100 & 2.438 & 2.384 \\
\hline circ-75km 8obj 1Man & 1.618 & 2.440 & 1.295 & 0.418 & 100 & 1.682 & 0.339 & 0.631 & 1.751 & 2.798 & 100 & 2.450 & 0.037 & 0.069 & 15.253 & 8.628 & 100 & 2.604 & 2.245 \\
\hline circ -80km 8obj 1Man & 2.165 & 3.275 & 1.768 & 0.392 & 100 & 1.365 & 0.331 & 0.607 & 1.726 & 2.802 & 100 & 2.463 & 0.035 & 0.067 & 15.207 & 8.620 & 100 & 2.642 & 2.157 \\
\hline circ-100km 8obj 1Man & 23.156 & 27.547 & 7.511 & 0.248 & 98.864 & 0.642 & 0.269 & 0.497 & 1.739 & 2.777 & 100 & 2.628 & 0.032 & 0.062 & 15.252 & 8.603 & 100 & 2.737 & 2.002 \\
\hline circ-150km 8obj 1Man & 90.000 & 90.000 & 0.000 & 0.000 & 0 & 0.028 & 0.201 & 0.374 & 1.666 & 2.711 & 100 & 2.878 & 0.028 & 0.055 & 15.114 & 8.560 & 100 & 2.892 & 1.932 \\
\hline circ-200km 8obj 1Man & 90.000 & 90.000 & 0.000 & 0.000 & 0 & 0.028 & 0.163 & 0.305 & 1.603 & 2.650 & 100 & 3.106 & 0.027 & 0.055 & 14.693 & 8.591 & 100 & 2.903 & 2.013 \\
\hline circ-300km 8obj 1Man & 90.000 & 90.000 & 0.000 & 0.000 & 0 & 0.028 & 0.130 & 0.241 & 1.447 & 2.401 & 100 & 3.341 & 0.026 & 0.053 & 14.368 & 8.552 & 100 & 2.942 & 2.104 \\
\hline circ-500km 8obj 1 Man & 90.000 & 90.000 & 0.000 & 0.000 & 0 & 0.028 & 0.173 & 0.309 & 0.754 & 1.576 & 100 & 2.309 & 0.026 & 0.051 & 13.741 & 8.516 & 100 & 2.938 & 1.758 \\
\hline circ $-550 \mathrm{~km}$ 8obj $1 \mathrm{Man}$ & 90.000 & 90.000 & 0.000 & 0.000 & 0 & 0.028 & 0.293 & 0.486 & 0.583 & 1.141 & 100 & 1.529 & 0.025 & 0.051 & 13.456 & 8.518 & 100 & 2.959 & 1.505 \\
\hline
\end{tabular}


Table 11 Cluster 1 Elliptical Phasing Maneuver Performance Results

\begin{tabular}{|c|c|c|c|c|c|c|c|c|c|c|c|c|c|c|c|c|c|c|c|}
\hline \multirow[b]{2}{*}{ Case } & \multicolumn{6}{|c|}{ Tier 1: $100 \mathrm{~km}$} & \multicolumn{6}{|c|}{ Tier 2: $600 \mathrm{~km}$} & \multicolumn{6}{|c|}{ Tier $3: 2400 \mathrm{~km}$} & \multirow[b]{2}{*}{$\begin{array}{l}\text { Total } \\
\text { Score }\end{array}$} \\
\hline & $\begin{array}{c}\text { MRT } \\
\text { (days) }\end{array}$ & $\begin{array}{l}\text { TAG } \\
\text { (days) }\end{array}$ & $\mathrm{PC}(\%)$ & \begin{tabular}{|c|} 
Meand \\
$(\mathrm{min})$ \\
\end{tabular} & CC (\%) & Score & $\begin{array}{l}\text { MRT } \\
\text { (days) }\end{array}$ & $\begin{array}{c}\text { TAG } \\
\text { (days) }\end{array}$ & $\mathrm{PC}(\%)$ & \begin{tabular}{|c|} 
Meand \\
$(\mathrm{min})$
\end{tabular} & $\mathrm{CC}(\%)$ & Score & $\begin{array}{l}\text { MRT } \\
\text { (days) }\end{array}$ & $\begin{array}{l}\text { TAG } \\
\text { (days) }\end{array}$ & PC (\%) & \begin{tabular}{|c|} 
Meand \\
$(\mathrm{min})$
\end{tabular} & $\mathrm{CC}(\%)$ & Score & \\
\hline circ -50km 8obj 0Man & 0.938 & 1.573 & 0.905 & 0.534 & 100 & 2.556 & 0.454 & 0.857 & 1.865 & 2.849 & 100 & 2.333 & 0.026 & 0.050 & 14.408 & 8.669 & 100 & 3.006 & 2.632 \\
\hline ecc ap550 pg50 8obj 1Man & 17.42 & 18.97 & 8.69 & 0.24 & 100 & 0.723 & 0.146 & 0.271 & 1.405 & 2.507 & 100 & 3.150 & 0.024 & 0.046 & 13.202 & 8.639 & 100 & 3.071 & 2.315 \\
\hline ecc ap550 pg100 8obj 1Man & 41.91 & 44.03 & 24.74 & 0.24 & 100 & .295 & 0.163 & 0.279 & 1.384 & 2.279 & 100 & 2.951 & 0.024 & 0.044 & 12.551 & 8.274 & 100 & 3.055 & 434 \\
\hline ecc ap550 pg200 8obj 1Man & 74.79 & 78.39 & 8.59 & 0.04 & 100 & .417 & 0.176 & 0.294 & 1.340 & 2.325 & 100 & 2.851 & 0.023 & 0.045 & 12.583 & 8.428 & 100 & 3.074 & 1114 \\
\hline ecc ap550 pg-50 8obj 1 Man & 5.082 & 8.383 & 5.416 & 0.345 & 100 & 0.926 & 0.177 & 0.305 & 1.557 & 2.483 & 100 & 2.971 & 0.025 & 0.048 & 14.097 & 9.077 & 100 & 3.084 & 327 \\
\hline ecc ap550 pg-75 8obj 1 Man & 5.761 & 8.303 & 4.283 & 0.329 & 100 & 0.845 & 0.191 & 0.328 & 1.747 & 2.723 & 100 & 3.035 & 0.022 & 0.042 & 12.376 & 7.960 & 100 & 3.115 & .332 \\
\hline ecc ap550 pg-100 8obj 1 Man & 5.270 & 7.781 & 5.046 & 0.316 & 100 & .891 & 0.181 & 0.295 & 1.575 & 2.446 & 100 & 2.977 & 0.022 & 0.043 & 12.474 & 7.998 & 100 & 3.114 & 328 \\
\hline ecc ap50 pg-550 8obj $1 \mathrm{Man}$ & 9.449 & 11.661 & 4.510 & 0.335 & 100 & 0.741 & 0.151 & 0.268 & 1.561 & 2.314 & 100 & 3.143 & 0.023 & 0.042 & 14.343 & 8.635 & 100 & 3.257 & .380 \\
\hline ecc ap75 pg-550 8obj 1 Man & 7.508 & 10.812 & 4.429 & 0.295 & 100 & 0.735 & 0.151 & 0.270 & 1.583 & 2.326 & 100 & 3.146 & 0.023 & 0.043 & 14.548 & 8.755 & 100 & 3.256 & 2.379 \\
\hline ecc ap100 pg-55 & 5.759 & 9.285 & 5.134 & 0.318 & 100 & 849 & 0.170 & 0.308 & 1.823 & 2.647 & 100 & 3.170 & 0.023 & 0.042 & 14.346 & 649 & 100 & 3.259 & 426 \\
\hline ecc ap200 pg-5 & 4.368 & 7.338 & 5.205 & 329 & 100 & 959 & 0.190 & 0.332 & 1.794 & 2.580 & 10 & 3.006 & 0.023 & 0.042 & 13.902 & 401 & 100 & 3.210 & 392 \\
\hline ecc ap-50 pg-550 8obj 1Man & 18.255 & 23.955 & 9.615 & 346 & 100 & 841 & 0.161 & 0.272 & 1.701 & 2.357 & 10 & .154 & 0.021 & 0.040 & 4.026 & 469 & & 320 & 439 \\
\hline ecc ap-75 pg-550 8obj 1 Man & 42.148 & 40.619 & 14.216 & 0.242 & 100 & 0.871 & 0.139 & 0.247 & 1.490 & 2.232 & 10 & 3.221 & 0.020 & 0.039 & 13.420 & 8.123 & 100 & 3.337 & 476 \\
\hline ecc ap-100 pg-550 8obj 1Man & 71.805 & 75.756 & 6.978 & & 94.318 & 0.416 & 0.154 & 0.273 & 1.624 & 2.451 & 100 & 3.180 & 0.022 & 0.042 & 14.431 & 8.714 & 100 & 3.306 & .301 \\
\hline ecc ap-20 & 90.000 & 90.000 & 0.000 & & 0 & 0.028 & 0.143 & 0.253 & 1.417 & 2. & 100 & 142 & 0.020 & 0.037 & 13.168 & 914 & 100 & 3.364 & 178 \\
\hline ecc ap550 pg0 8obj 1Man & 8.611 & 13.313 & 5.893 & 0.335 & 100 & 0.790 & 0.175 & 0.295 & 1.511 & 2.403 & 100 & 2.957 & 0.026 & 0.047 & 13.729 & 8.900 & 100 & 3.051 & .266 \\
\hline ecc ap0 pg-550 8obj 1Man & 13.086 & 16.701 & 4.650 & 297 & 100 & 0.642 & 0.159 & 0.275 & 1.607 & 2.412 & 10 & 3.130 & 0.021 & 0.038 & 13.270 & 7.992 & 100 & 3.308 & .360 \\
\hline ecc ap $200 \mathrm{pg}$ & 8.689 & 13.218 & 6.729 & & & 805 & 0.209 & 0.368 & 1.729 & & & 32 & 0.024 & 0.047 & 13.948 & & 00 & 05 & 247 \\
\hline ecc ap200 & 25.203 & 31.590 & 18.600 & 0.441 & 100 & 1.267 & 0.202 & 0.353 & 1.791 & 2.586 & 100 & 2.925 & 0.025 & 0.048 & 14.218 & 9.094 & 100 & 3.100 & 431 \\
\hline ecc ap200 pg-50 8obj 1Man & 1.574 & 2.539 & 1.596 & 0.586 & & 1.849 & 0.346 & 0.615 & 1.838 & 2.594 & & 424 & 0.024 & 0.046 & 2.907 & 16 & 00 & 12 & 428 \\
\hline ecc ap200 pg-75 8obj 1Man & 1.659 & 2.703 & 1.066 & 0.621 & 100 & 1.793 & 0.416 & 0.736 & 1.827 & 2.598 & 100 & 2.301 & 0.029 & 0.054 & 14.817 & 9.286 & 100 & 2.951 & 2.348 \\
\hline ecc ap200 pg-100 8obj 1Man & 2.366 & 3.401 & 4.651 & 0.624 & 100 & 1.653 & 0.518 & 0.924 & 1.842 & 2.629 & 100 & 2.197 & 0.030 & 0.056 & 14.504 & 9.074 & 100 & 2.861 & 2.237 \\
\hline ecc ap50 pg-200 & 1.774 & 2.531 & 6.232 & & 10 & 1.9 & 0.323 & 0.580 & 1.924 & & & & 0.024 & 0.046 & 14.073 & & 00 & & 532 \\
\hline ecc ap75 pg-200 & 1.554 & 2.455 & 4.030 & 0.555 & 100 & 1.946 & 0.362 & 0.638 & 1.819 & 2.531 & 100 & 2.366 & 0.026 & 0.046 & 13.973 & .547 & 100 & 3.039 & .450 \\
\hline ecc ap100 pg-200 8obj 1Man & 1.769 & 2.804 & 4.255 & 0.688 & & 1.931 & 0.493 & 0.884 & 2.179 & 2.907 & 10 & 2.467 & 0.026 & 0.049 & 14.038 & 8.576 & 100 & 3.010 & .469 \\
\hline ecc ap-50 pg-200 8obj 1Man & 5.653 & 6.902 & 17.609 & 0.384 & 100 & 1.479 & 0.234 & 0.414 & 2.057 & 2.886 & 100 & 2.970 & 0.022 & 0.042 & 13.604 & 340 & 100 & 3.213 & 2.554 \\
\hline ecc ap-75 pg-200 8obj 1Man & 13.748 & 15.303 & 15.690 & 0.574 & \begin{tabular}{|l|l|}
97.727 \\
\end{tabular} & 1.362 & 0.202 & 0.358 & 1.946 & 2.677 & & 3.020 & 0.023 & 0.042 & 14.063 & 09 & 00 & 3.222 & .535 \\
\hline ecc ap-10 & 25.301 & 25.991 & 7.378 & & 97.727 & 0.488 & 0.19 & 0.335 & 1.937 & 2.659 & & 90 & 0.022 & 0.042 & 14.071 & 02 & & 3.261 & 80 \\
\hline ecc ap200 & 2.062 & 3.145 & 3.874 & 0.585 & 100 & 1.679 & 0.287 & 0.498 & 1.879 & 2.676 & 10 & .630 & 0.026 & 0.049 & 14.499 & 9.146 & 100 & 3.061 & .457 \\
\hline ecc ap0 pg-200 8obj 1Man & 2.403 & 3.607 & 3.515 & 0.653 & 100 & 1.602 & 0.277 & 0.493 & 2.056 & 2.884 & 100 & 2.800 & 0.024 & 0.046 & 14.756 & 9.030 & 100 & 3.181 & 2.528 \\
\hline ecc ap100 pg50 8obj 1Man & 1.374 & 2.209 & 1.902 & 0.641 & 100 & 2.093 & 0.364 & 0.641 & 1.916 & 2.710 & 100 & 2.466 & 0.027 & 0.050 & 14.148 & 8.916 & 100 & 3.002 & 2.521 \\
\hline ecc ap100 pg75 8obj 1Man & 2.743 & 4.310 & 4.060 & & 100 & 1.345 & 0.300 & 0.535 & 1.855 & 2.591 & 10 & 2.5 & 0.025 & 0.047 & 13.540 & 40 & 100 & 3.019 & 2.301 \\
\hline ecc ap100 pg-50 8obj 1Man & 2.225 & 3.669 & 0.656 & & & & 1.38 & 2.45 & 2.086 & & 10 & & 0.044 & 0.083 & 14.668 & & & 2.450 & 2.095 \\
\hline ecc ap100 pg-75 8obj 1Man & 8.123 & 11.152 & 0.587 & & & 1.0 & 4.228 & 6.800 & 2.003 & 2.673 & & 66 & 0.100 & 0.186 & 14.870 & 8.777 & 100 & 1.924 & 1.624 \\
\hline ecc ap50 pg-100 8obj 1Man & 3.007 & 3.007 & 0.841 & 0.854 & 100 & 1.699 & 0.975 & 1.637 & 2.010 & 2.650 & 100 & 2.063 & 0.034 & 0.059 & 13.971 & 8.507 & 100 & 2.671 & 2.144 \\
\hline ecc ap75 pg-100 8obj 1Man & 17.757 & 6.906 & 0.886 & 1.030 & 100 & 1.316 & 3.577 & 3.196 & 2.182 & 2.880 & 100 & 2.060 & 0.062 & 0.070 & 15.870 & 9.513 & 100 & 2.493 & 1.956 \\
\hline ecc ap-50 pg-100 8obj 1 Man & 1.543 & 1.787 & 1.660 & 0.527 & 100 & 2.067 & 0.305 & 0.548 & 1.841 & 2.567 & 10 & 2.509 & 0.025 & 0.049 & 14.918 & 9.166 & 100 & 3.114 & 2.563 \\
\hline
\end{tabular}


An interesting area of note is how the phasing maneuver impacted the performance of each Tier differently. For both circular and elliptical constellations, the smaller the range constraint is, the better impact phasing maneuvers have on the performance metrics. Circular constellations have a 9\% improved Tier 1 performance with phasing maneuvers, while Tier 2 and 3 performance decreased by $4.4 \%$ and $15.2 \%$, respectively. Similarly, elliptical constellations have a $13.5 \%$, $1,5 \%$, and $0.4 \%$ improved performance for Tiers 1,2 , and 3 . This suggests that if increased weight is placed on lower Tiers, for example if a mission were focused more on minimizing the range at closest approach, phasing maneuvers could offer significantly better performance improvements than these results indicate. Especially since it has been shown that Tier 1 has a larger impact on the total score than Tiers 2 and 3.

A potential improvement that could be implemented in future research would be to calculate a different phasing angle for each maneuver that will position the OBS within each clusters' altitude range for the majority of the phasing orbit. The method used in this research phased each OBS by 10 degrees regardless of the constellation offset, which is what led to most observer satellites having no debris conjunctions while phasing. If the phase angle were targeted based on OBS offset, the phasing orbit could be tuned so phasing OBS pass through the cluster altitude range for the majority of the phasing orbit, hopefully increasing conjunction rate while phasing.

\section{RAAN Maneuver Cases}

As with the phasing maneuver, the RAAN maneuvering cases are only analyzed for Cluster 1. Large portions of the debris clusters surface area are unmonitored by OBSs, meaning conjunctions near the minimum and maximum latitudes are relied on for many of the RSOs conjunctions. RAAN maneuvers are intended to improve the spherical coverage of the clusters. Figure 23 highlights this gap in coverage, comparing a non-maneuvering constellation on the left with a RAAN- 
maneuvering constellation on the right. The orbit path for all maneuvering observer satellites are shown in green, to highlight the improved spherical coverage. RAAN maneuvers are executed by performing a 5 degree $\Delta \Omega$ shift every 15 days of orbit propagation, to rotate each orbit a total of 120 degrees over a yearlong simulation.

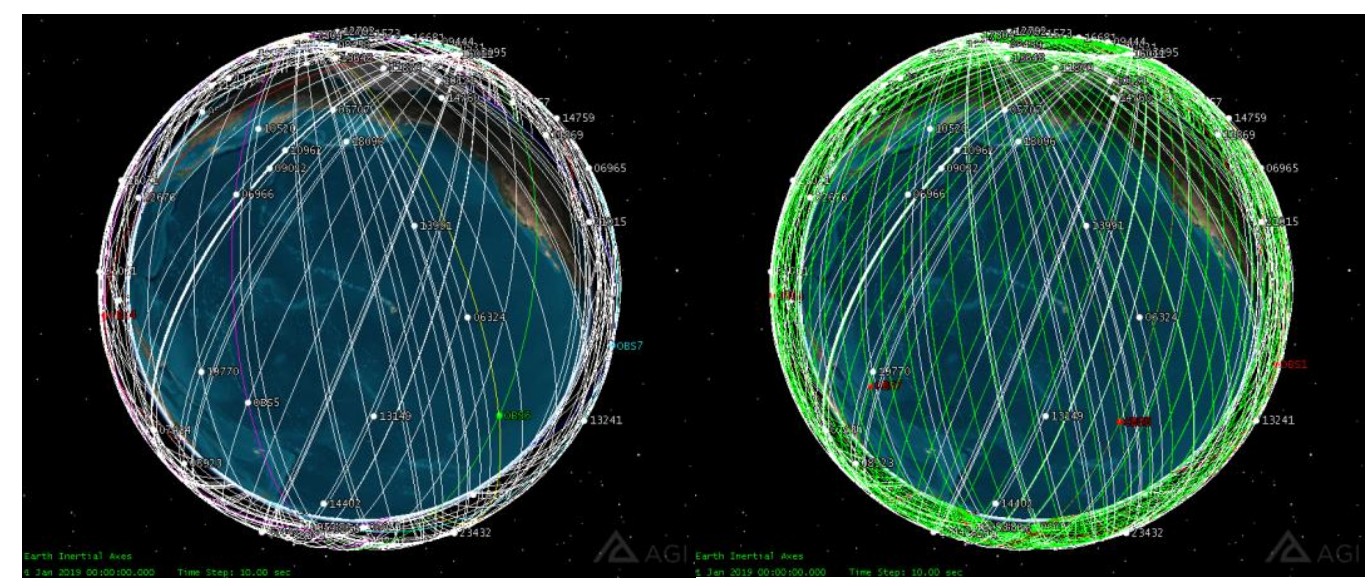

Fig. 23 Spherical Coverage Gaps

The results show that RAAN maneuvering cases performed similar to the phasing maneuver cases, with mixed results but an overall worse performance than static cases for both circular and elliptical constellations. RAAN maneuvers outperformed phasing maneuver cases, but ultimately the static circular cases provide the best performance based on the grading metrics. With RAAN maneuvers, observer satellites do not spend time on phasing orbits like they do with phasing maneuvers, reducing the impact on conjunction gaps. Figures 24-25 show the total score for each Tier. Tables 12-13 contain the full performance results for circular and elliptical cases.

Comparing the static and maneuvering cases for circular constellation designs, the static cases have an average score of 2.13 with a standard deviation of 0.33 , while the maneuvering cases have an average score of 2.13 with a standard deviation of 0.29 . The average results are equivalent for the static and maneuvering circular cases, but the best overall case remains the static circular -50 $\mathrm{km}$ offset constellation with a score of 2.63. The best performing maneuvering case is the $-80 \mathrm{~km}$ 


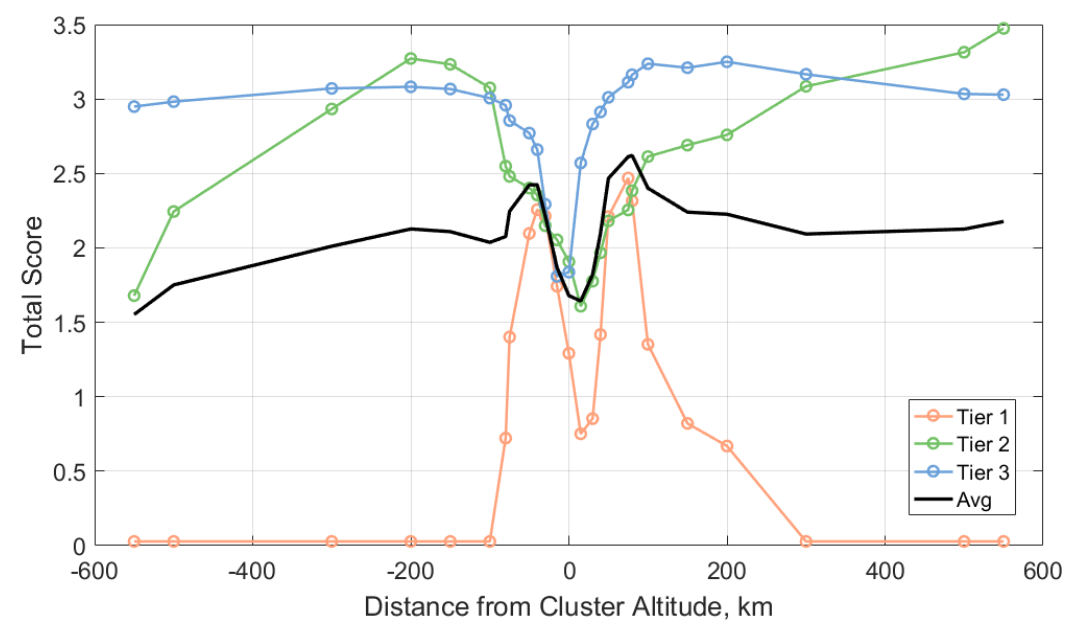

Fig. 24 Cluster 1 Circular RAAN Maneuver Total Score

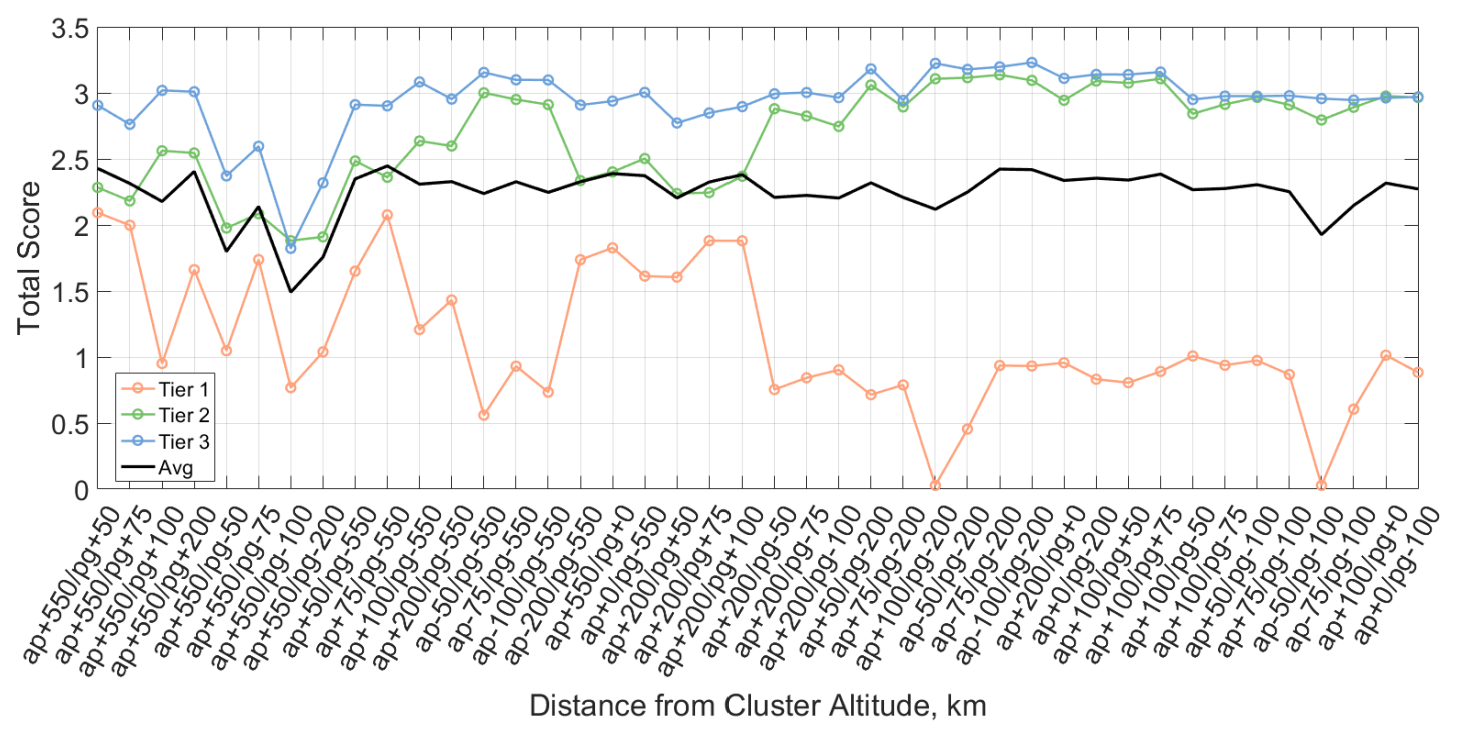

Fig. 25 Cluster 1 Elliptical RAAN Maneuver Total Score

offset with a total score of 2.62. For the elliptical constellations, the static cases have an average score of 2.28 with a standard deviation of 0.19 , while the maneuvering cases have an average score of 2.25 with a standard deviation of 0.19 . There are minor differences in all of the metrics between the static and maneuvering cases, but the results are virtually identical. RAAN maneuvering cases, though, require a large $\Delta V$ of roughly $650 \mathrm{~m} / \mathrm{s}$ per maneuver, meaning the maneuvering constellations require a significant improvement in performance metrics to be considered successful. 
Table 12 Cluster 1 Circular RAAN Maneuver Performance Results

\begin{tabular}{|c|c|c|c|c|c|c|c|c|c|c|c|c|c|c|c|c|c|c|c|}
\hline \multirow[b]{2}{*}{ Case } & \multicolumn{6}{|c|}{ Tier 1: $100 \mathrm{~km}$} & \multicolumn{6}{|c|}{ Tier 2: $600 \mathrm{~km}$} & \multicolumn{6}{|c|}{ Tier 3: $2400 \mathrm{~km}$} & \multirow{2}{*}{$\begin{array}{l}\text { Total } \\
\text { Score }\end{array}$} \\
\hline & $\begin{array}{l}\text { MRT } \\
\text { (days) }\end{array}$ & $\begin{array}{l}\text { TAG } \\
\text { (days) }\end{array}$ & PC (\%) & $\begin{array}{c}\text { MeanD } \\
(\mathrm{min})\end{array}$ & CC (\%) & Score & $\begin{array}{l}\text { MRT } \\
\text { (days) }\end{array}$ & $\begin{array}{c}\text { TAG } \\
\text { (days) }\end{array}$ & PC (\%) & \begin{tabular}{|c} 
MeanD \\
$(\mathrm{min})$
\end{tabular} & $\mathrm{CC}(\%)$ & Score & $\begin{array}{c}\text { MRT } \\
\text { (days) }\end{array}$ & $\begin{array}{c}\text { TAG } \\
\text { (days) }\end{array}$ & PC (\%) & $\begin{array}{c}\text { MeanD } \\
(\mathrm{min})\end{array}$ & CC (\%) & Score & \\
\hline circ - $50 \mathrm{~km}$ 8obj 0Man & 0.938 & 1.573 & 0.905 & 0.534 & 100 & 2.556 & 0.454 & 0.857 & 1.865 & 2.849 & 100 & 2.333 & 0.026 & 0.050 & 14.408 & 8.669 & 100 & 3.006 & 2.632 \\
\hline circ $+550 \mathrm{~km} 8$ obj $2 \mathrm{Man}$ & 90.00 & 90.00 & 0.00 & 0.00 & 0 & 0.028 & 0.254 & 0.469 & 0.609 & 1.312 & 100 & 1.679 & 0.025 & 0.048 & 12.305 & 8.497 & 100 & 2.949 & 1.552 \\
\hline circ $+500 \mathrm{~km} 8 \mathrm{obj} 2 \mathrm{Man}$ & 90.00 & 90.00 & 0.00 & 0.00 & 0 & 0.028 & 0.185 & 0.344 & 0.762 & 1.728 & 100 & 2.244 & 0.025 & 0.048 & 12.517 & 8.532 & 100 & 2.982 & 1.751 \\
\hline circ $+300 \mathrm{~km} 8 \mathrm{obj} 2 \mathrm{Man}$ & 90.00 & 90.00 & 0.00 & 0.00 & 0 & 0.028 & 0.146 & 0.275 & 1.172 & 2.210 & 100 & 2.933 & 0.024 & 0.046 & 13.141 & 8.654 & 100 & 3.071 & 2.011 \\
\hline circ $+200 \mathrm{~km}$ 8obj $2 \mathrm{Man}$ & 90.00 & 90.00 & 0.00 & 0.00 & 0 & 0.028 & 0.136 & 0.257 & 1.440 & 2.505 & 100 & 3.272 & 0.024 & 0.046 & 13.449 & 8.682 & 100 & 3.082 & 2.127 \\
\hline circ $+150 \mathrm{~km}$ 8obj 2Man & 90.000 & 90.000 & 0.000 & 0.000 & 0 & 0.028 & 0.147 & 0.278 & 1.558 & 2.627 & 100 & 3.233 & 0.025 & 0.047 & 13.609 & 8.697 & 100 & 3.067 & 2.109 \\
\hline circ $+100 \mathrm{~km}$ 8obj 2Man & 90.000 & 90.000 & 0.000 & 0.000 & 0 & 0.028 & 0.169 & 0.319 & 1.618 & 2.708 & 100 & 3.075 & 0.026 & 0.049 & 13.793 & 8.695 & 100 & 3.006 & 2.037 \\
\hline circ $+80 \mathrm{~km}$ 8obj $2 \mathrm{Man}$ & 7.033 & 9.281 & 2.817 & 0.314 & 100 & 0.722 & 0.300 & 0.559 & 1.747 & 2.819 & 100 & 2.549 & 0.027 & 0.051 & 13.864 & 8.700 & 100 & 2.958 & 2.076 \\
\hline circ $+75 \mathrm{~km}$ 8obj $2 \mathrm{Man}$ & 2.056 & 3.333 & 2.220 & 0.395 & 100 & 1.401 & 0.326 & 0.619 & 1.760 & 2.811 & 100 & 2.480 & 0.028 & 0.055 & 13.881 & 8.729 & 100 & 2.855 & 2.246 \\
\hline circ $+50 \mathrm{~km} 8 \mathrm{obj} 2 \mathrm{Man}$ & 1.148 & 2.012 & 1.543 & 0.449 & 100 & 2.097 & 0.370 & 0.696 & 1.762 & 2.830 & 100 & 2.402 & 0.031 & 0.057 & 13.908 & 8.671 & 100 & 2.771 & 2.423 \\
\hline $\mathrm{circ}+40 \mathrm{~km}$ 8obj $2 \mathrm{Man}$ & 1.037 & 1.814 & 1.085 & 0.454 & 100 & 2.257 & 0.388 & 0.743 & 1.743 & 2.822 & 100 & 2.355 & 0.033 & 0.064 & 14.018 & 8.691 & 100 & 2.660 & 2.424 \\
\hline circ $+30 \mathrm{~km}$ 8obj $2 \mathrm{Man}$ & 1.108 & 2.025 & 0.713 & 0.578 & 100 & 2.213 & 0.646 & 1.195 & 1.788 & 2.868 & 100 & 2.149 & 0.043 & 0.103 & 13.978 & 8.581 & 100 & 2.293 & 2.218 \\
\hline circ $+15 \mathrm{~km}$ 8obj $2 \mathrm{Man}$ & 1.519 & 2.704 & 0.341 & 0.545 & 100 & 1.742 & 0.853 & 1.628 & 1.807 & 2.845 & 100 & 2.054 & 0.108 & 0.168 & 13.503 & 8.156 & 100 & 1.809 & 1.868 \\
\hline circ $+0 \mathrm{~km}$ 8obj 2Man & 2.496 & 4.664 & 0.220 & 0.587 & 100 & 1.292 & 1.556 & 2.947 & 1.769 & 2.837 & 100 & 1.907 & 0.154 & 0.133 & 13.712 & 8.316 & 100 & 1.837 & 1.679 \\
\hline circ - $15 \mathrm{~km}$ 8obj 2Man & 8.884 & 12.741 & 0.424 & 0.520 & 100 & 0.751 & 5.788 & 7.177 & 1.594 & 2.486 & 100 & 1.608 & 0.037 & 0.066 & 13.944 & 8.597 & 100 & 2.570 & 1.643 \\
\hline circ -30km 8obj 2Man & 6.212 & 8.771 & 0.111 & 0.533 & 100 & 0.853 & 3.090 & 4.632 & 1.789 & 2.619 & 100 & 1.776 & 0.030 & 0.054 & 14.077 & 8.648 & 100 & 2.832 & 1.820 \\
\hline circ -40km 8obj 2Man & 2.178 & 3.702 & 0.291 & 0.568 & 100 & 1.418 & 1.091 & 2.007 & 1.781 & 2.792 & 100 & 1.967 & 0.028 & 0.051 & 14.114 & 8.648 & 100 & 2.913 & 2.099 \\
\hline circ - $50 \mathrm{~km}$ 8obj $2 \mathrm{Man}$ & 1.122 & 1.952 & 0.522 & 0.563 & 100 & 2.210 & 0.610 & 1.116 & 1.822 & 2.844 & 100 & 2.181 & 0.026 & 0.049 & 14.145 & 8.646 & 100 & 3.011 & 2.467 \\
\hline circ $-75 \mathrm{~km}$ 8obj 2Man & 0.954 & 1.649 & 0.702 & 0.519 & 100 & 2.469 & 0.475 & 0.895 & 1.776 & 2.804 & 100 & 2.255 & 0.024 & 0.046 & 14.154 & 8.627 & 100 & 3.113 & 2.612 \\
\hline circ -80km 8obj 2Man & 0.996 & 1.839 & 0.799 & 0.502 & 100 & 2.316 & 0.400 & 0.754 & 1.835 & 2.825 & 100 & 2.384 & 0.023 & 0.045 & 13.938 & 8.616 & 100 & 3.163 & 2.621 \\
\hline circ $-100 \mathrm{~km}$ 8obj 2 Man & 2.083 & 3.293 & 1.632 & 0.369 & 100 & 1.352 & 0.280 & 0.526 & 1.784 & 2.796 & 100 & 2.613 & 0.022 & 0.043 & 13.892 & 8.593 & 100 & 3.237 & 2.401 \\
\hline circ $-150 \mathrm{~km}$ 8obj 2 Man & 6.189 & 8.759 & 3.582 & 0.355 & 98.864 & 0.821 & 0.254 & 0.481 & 1.773 & 2.786 & 100 & 2.690 & 0.021 & 0.046 & 13.682 & 8.544 & 100 & 3.210 & 2.240 \\
\hline circ -200km 8obj 2Man & 22.025 & 27.100 & 8.219 & 0.243 & 98.864 & 0.669 & 0.237 & 0.445 & 1.771 & 2.776 & 100 & 2.759 & 0.021 & 0.043 & 13.159 & 8.587 & 100 & 3.250 & 2.226 \\
\hline circ-300km 8obj 2Man & 90.000 & 90.000 & 0.000 & 0.000 & 0 & 0.028 & 0.175 & 0.331 & 1.741 & 2.722 & 100 & 3.085 & 0.022 & 0.044 & 12.828 & 8.512 & 100 & 3.166 & 2.093 \\
\hline circ - $500 \mathrm{~km}$ 8obj 2 Man & 90.000 & 90.000 & 0.000 & 0.000 & 0 & 0.028 & 0.145 & 0.273 & 1.671 & 2.643 & 100 & 3.314 & 0.024 & 0.047 & 12.823 & 8.497 & 100 & 3.034 & 2.126 \\
\hline circ - $550 \mathrm{~km}$ 8obj $2 \mathrm{Man}$ & 90.000 & 90.000 & 0.000 & 0.000 & 0 & 0.028 & 0.130 & 0.241 & 1.629 & 2.551 & 100 & 3.474 & 0.023 & 0.049 & 12.567 & 8.503 & 100 & 3.029 & 2.177 \\
\hline
\end{tabular}

Table 13 Cluster 1 Elliptical RAAN Maneuver Performance Results

\begin{tabular}{|c|c|c|c|c|c|c|c|c|c|c|c|c|c|c|c|c|c|c|c|}
\hline \multirow[b]{2}{*}{ Case } & \multicolumn{6}{|c|}{ Tier 1: $100 \mathrm{~km}$} & \multicolumn{6}{|c|}{ Tier 2: $600 \mathrm{~km}$} & \multicolumn{6}{|c|}{ Tier $3: 2400 \mathrm{~km}$} & \multirow[b]{2}{*}{$\begin{array}{l}\text { Total } \\
\text { Score }\end{array}$} \\
\hline & $\begin{array}{l}\text { MRT } \\
\text { (days) }\end{array}$ & $\begin{array}{c}\text { TAG } \\
\text { (days) }\end{array}$ & PC (\%) & $\begin{array}{c}\text { MeanD } \\
\text { (min) }\end{array}$ & $\mathrm{CC}(\%)$ & Score & $\begin{array}{l}\text { MRT } \\
\text { (days) }\end{array}$ & $\begin{array}{c}\text { TAG } \\
\text { (days) }\end{array}$ & PC (\%) & $\begin{array}{c}\text { MeanD } \\
\text { (min) }\end{array}$ & $\mathrm{CC}(\%)$ & Score & $\begin{array}{l}\text { MRT } \\
\text { (days) }\end{array}$ & $\begin{array}{c}\text { TAG } \\
\text { (days) }\end{array}$ & PC (\%) & $\begin{array}{c}\text { MeanD } \\
\text { (min) }\end{array}$ & $\mathrm{CC}(\%)$ & Score & \\
\hline circ -50km 8obj 0Man & 0.938 & 1.573 & 0.905 & 0.534 & 100 & 2.556 & 0.454 & 0.857 & 1.865 & 2.849 & 100 & 2.333 & 0.026 & 0.050 & 14.408 & 8.669 & 100 & 3.006 & 2.632 \\
\hline ecc ap550 pg50 8obj 2Man & 13.81 & 20.66 & 11.63 & 0.28 & 100 & 0.884 & 0.164 & 0.298 & 1.364 & 2.513 & 100 & 2.965 & 0.026 & 0.047 & 12.922 & 8.456 & 100 & 2.970 & 2.273 \\
\hline ecc ap550 pg75 8obj 2Man & 20.92 & 27.91 & 19.12 & 0.14 & 100 & 1.014 & 0.161 & 0.294 & 1.358 & 2.481 & 100 & 2.976 & 0.027 & 0.049 & 13.435 & 8.853 & 100 & 2.962 & 2.317 \\
\hline ecc ap550 pg100 8obj 2Man & 63.69 & 64.46 & 12.50 & 0.06 & 100 & 0.606 & 0.159 & 0.292 & 1.252 & 2.331 & 100 & 2.891 & 0.026 & 0.045 & 12.186 & 8.034 & 100 & 2.946 & 2.148 \\
\hline ecc ap550 pg 200 8obj 2Man & 90.00 & 90.00 & 0.00 & 0.00 & 0 & 0.028 & 0.170 & 0.306 & 1.203 & 2.360 & 100 & 2.795 & 0.026 & 0.047 & 12.592 & 8.434 & 100 & 2.957 & 1.927 \\
\hline ecc ap550 pg-50 8obj 2Man & 5.527 & 8.652 & 5.349 & 0.309 & 100 & 0.868 & 0.177 & 0.326 & 1.441 & 2.598 & 100 & 2.909 & 0.026 & 0.047 & 13.009 & 8.377 & 100 & 2.979 & 2.252 \\
\hline ecc ap550 pg-75 8obj 2Man & 4.403 & 6.763 & 5.943 & 0.297 & 100 & 0.974 & 0.172 & 0.315 & 1.462 & 2.608 & 100 & 2.966 & 0.027 & 0.048 & 13.439 & 8.644 & 100 & 2.975 & 2.305 \\
\hline ecc ap550 pg-100 8obj 2Man & 4.318 & 6.722 & 5.061 & 0.290 & 100 & 0.938 & 0.181 & 0.318 & 1.466 & 2.574 & 100 & 2.913 & 0.027 & 0.049 & 13.614 & 8.729 & 100 & 2.976 & 2.276 \\
\hline ecc ap550 pg-200 8obj $2 \mathrm{Man}$ & 3.727 & 6.178 & 3.885 & 0.355 & 100 & 1.008 & 0.197 & 0.369 & 1.565 & 2.684 & 100 & 2.842 & 0.028 & 0.049 & 13.816 & 8.782 & 100 & 2.950 & 2.267 \\
\hline ecc ap50 pg-550 8obj $2 \mathrm{Man}$ & 7.558 & 12.312 & 6.073 & 0.406 & 100 & 0.891 & 0.152 & 0.289 & 1.508 & 2.492 & 100 & 3.107 & 0.023 & 0.040 & 13.221 & 7.959 & 100 & 3.158 & 2.385 \\
\hline ecc ap75 pg-550 8obj 2Man & 6.732 & 10.541 & 5.063 & 0.322 & 100 & .806 & 0.164 & 0.313 & 1.590 & 2.640 & 100 & 3.075 & 0.025 & 0.043 & 14.125 & 501 & 100 & 3.139 & 2.340 \\
\hline ecc ap100 pg-550 8obj 2Man & 5.819 & 9.298 & 4.672 & 0.322 & 100 & 0.832 & 0.151 & 0.293 & 1.502 & 2.466 & 100 & 3.090 & 0.024 & 0.043 & 14.058 & 8.475 & 100 & 3.140 & 2.354 \\
\hline ecc ap $200 \mathrm{pg}-550 \mathrm{8obj} 2 \mathrm{Man}$ & 4.744 & 7.331 & 4.188 & 0.387 & 100 & 0.957 & 0.191 & 0.358 & 1.676 & 2.724 & 100 & 2.944 & 0.024 & 0.041 & 12.974 & 7.841 & 100 & 3.110 & 2.337 \\
\hline ecc ap-50 pg-550 8obj $2 \mathrm{Man}$ & 18.289 & 22.768 & 14.059 & 0.250 & 100 & 0.932 & 0.153 & 0.292 & 1.490 & 2.523 & 100 & 3.095 & 0.021 & 0.039 & 12.796 & 7.726 & 100 & 3.230 & 2.419 \\
\hline ecc ap-75 pg-550 8obj $2 \mathrm{Man}$ & 35.161 & 37.701 & 16.180 & 0.220 & 100 & 936 & 0.143 & 0.274 & 1.425 & 2.414 & 100 & 3.137 & 0.023 & 0.041 & 13.574 & 217 & 100 & 3.197 & 2.423 \\
\hline ecc ap-100 pg-550 8obj $2 \mathrm{Man}$ & 72.548 & 72.161 & 9.769 & 0.026 & 96.591 & 0.455 & 0.147 & 0.278 & 1.430 & 2.441 & 100 & 3.115 & 0.024 & 0.044 & 14.593 & 8.812 & 100 & 3.178 & 2.249 \\
\hline ecc ap-200 pg-550 8obj $2 \mathrm{Man}$ & 90.000 & 90.000 & 0.000 & 0.000 & 0 & 0.028 & 0.131 & 0.251 & 1.211 & 2.159 & 100 & 3.107 & 0.022 & 0.039 & 13.218 & 7.944 & 100 & 3.224 & 2.119 \\
\hline ecc ap550 pg0 8obj $2 \mathrm{Man}$ & 8.583 & 13.079 & 5.827 & 0.334 & 100 & 0.789 & 0.173 & 0.313 & 1.388 & 2.495 & 100 & 2.894 & 0.027 & 0.048 & 13.226 & 8.574 & 100 & 2.944 & 2.209 \\
\hline ecc ap0 pg-550 8obj 2Man & 18.439 & 18.343 & 4.977 & 0.388 & 100 & 0.715 & 0.161 & 0.299 & 1.510 & 2.561 & 100 & 3.060 & 0.023 & 0.039 & 13.077 & 7.876 & 100 & 3.182 & 2.319 \\
\hline ecc ap200 pg50 8obj 2Man & 4.159 & 6.805 & 4.551 & 0.270 & 100 & 0.903 & 0.241 & 0.458 & 1.734 & 2.864 & 100 & 2.746 & 0.028 & 0.049 & 13.922 & 8.866 & 100 & 2.964 & 2.204 \\
\hline ecc ap200 pg75 8obj 2Man & 8.196 & 12.893 & 8.936 & 0.252 & 100 & 0.843 & 0.229 & 0.432 & 1.753 & 2.900 & 100 & 2.825 & 0.025 & 0.045 & 12.858 & 8.190 & 100 & 3.003 & 2.224 \\
\hline ecc ap200 pg100 8obj 2Man & 33.238 & 39.658 & 15.214 & 0.074 & 100 & 0.754 & 0.209 & 0.391 & 1.715 & 2.799 & 100 & 2.880 & 0.026 & 0.047 & 13.332 & 8.528 & 100 & 2.993 & 2.209 \\
\hline ecc ap200 pg-50 8obj 2Man & 1.429 & 2.425 & 1.436 & 0.532 & 100 & 1.880 & 0.342 & 0.653 & 1.685 & 2.689 & 100 & 2.369 & 0.029 & 0.052 & 13.972 & 8.786 & 100 & 2.896 & 2.381 \\
\hline ecc ap200 pg-75 8obj 2Man & 1.475 & 2.566 & 1.365 & 0.595 & 100 & 1.881 & 0.460 & 0.880 & 1.730 & 2.800 & 100 & 2.245 & 0.031 & 0.055 & 14.420 & .037 & 100 & 2.848 & 2.325 \\
\hline ecc ap200 pg-100 8obj $2 \mathrm{Man}$ & 2.166 & 3.380 & 1.140 & 0.680 & 100 & 1.605 & 0.549 & 1.051 & 1.812 & 2.923 & 100 & 2.238 & 0.034 & 0.059 & 14.596 & .132 & 100 & 2.772 & 2.205 \\
\hline ecc ap50 pg-200 8obj 2Man & 1.871 & 2.751 & 1.616 & 0.489 & 100 & 1.613 & 0.324 & 0.625 & 1.794 & 2.839 & 100 & 2.503 & 0.026 & 0.047 & 13.908 & 8.512 & 100 & 3.004 & 2.373 \\
\hline ecc ap75 pg-200 8obj 2Man & 1.494 & 2.551 & 1.192 & & 100 & & 0.390 & 0.737 & 1.817 & 2.858 & & 2.4 & 0.027 & 0.045 & & & & 2.938 & 2.389 \\
\hline ecc ap100 pg-200 8obj 2Man & 1.664 & 2.914 & 1.461 & 0.592 & 100 & 1.737 & 0.462 & 0.889 & 1.895 & 2.858 & 100 & 2.336 & 0.027 & 0.047 & 13.080 & 7.992 & 100 & 2.908 & 2.327 \\
\hline есc ap-50 pg-200 8obj 2Man & 8.414 & 11.839 & 5.665 & 0.268 & 100 & 0.734 & 0.202 & 0.381 & 1.744 & 2.747 & 100 & 2.911 & 0.024 & 0.042 & 13.209 & .098 & 100 & 3.098 & 2.247 \\
\hline ecc ap-75 pg-200 8obj 2Man & 19.797 & 23.975 & 14.088 & 0.256 & 100 & 0.931 & 0.200 & 0.381 & 1.789 & 2.781 & & 2.950 & 0.026 & 0.045 & 14.496 & 8.874 & 100 & 3.099 & 2.327 \\
\hline ecc ap-100 pg-200 8obj $2 \mathrm{Man}$ & 67.250 & 67.477 & 9.193 & 0.155 & 97.727 & 0.560 & 0.179 & 0.339 & 1.696 & 2.631 & & 3.00 & 0.023 & 0.041 & 13.209 & 8.075 & 100 & 3.155 & 2.238 \\
\hline ecc ap $200 \mathrm{pg} 08 \mathrm{obj} 2 \mathrm{Man}$ & 2.046 & 3.320 & 2.246 & 0.421 & 100 & 1.432 & 0.293 & 0.546 & 1.779 & 2.864 & 100 & 2.598 & 0.028 & 0.050 & 14.185 & 8.948 & 100 & 2.953 & 2.328 \\
\hline ecc ap0 pg-200 8obj 2Man & 3.075 & 3.891 & 2.457 & 0.412 & 100 & 1.208 & 0.242 & 0.461 & 1.665 & 2.640 & 100 & 2.636 & 0.024 & 0.043 & 13.227 & 8.094 & 100 & 3.083 & 2.309 \\
\hline ecc ap100 pg50 8obj 2Man & 1.162 & 2.000 & 1.129 & & & & 0.389 & 0.741 & 1.756 & 2.824 & & 2.361 & 0.030 & 0.053 & 14.465 & 116 & 100 & 2.902 & 2.447 \\
\hline ecc ap100 pg75 8obj 2Man & 1.573 & 2.699 & 1.805 & 0.410 & 100 & 1.650 & 0.298 & 0.569 & 1.706 & 2.695 & & 2.485 & 0.027 & 0.048 & 13.191 & 8.320 & 100 & 2.911 & 2.349 \\
\hline ecc ap100 pg-50 8obj 2Man & 5.126 & 6.892 & 0.165 & 0.640 & 100 & 1.039 & 1.236 & 2.353 & 1.731 & 2.779 & 100 & 1.910 & 0.043 & 0.077 & 13.012 & 8.085 & 100 & 2.319 & 1.756 \\
\hline ecc ap100 pg-75 8obj 2Man & 9.424 & 12.668 & 0.308 & 0.547 & 100 & 0.768 & 4.326 & 7.472 & 1.902 & 2.869 & 100 & 1.881 & 0.103 & 0.181 & 13.902 & 8.206 & 100 & 1.823 & 1.491 \\
\hline ecc ap50 pg-100 8obj 2Man & 1.614 & 2.779 & 0.668 & & & & 1.010 & 1.820 & 1.931 & 2.879 & & & 0.03 & 0.063 & 14.270 & 8.689 & 100 & 2.596 & 2.140 \\
\hline ecc ap75 pg-100 8obj 2Man & 7.615 & 5.071 & 0.439 & 0.615 & 100 & 1.048 & 3.472 & 3.332 & 1.966 & 2.933 & 100 & 1.977 & 0.063 & 0.067 & 14.452 & 8.663 & 100 & 2.371 & 1.799 \\
\hline ecc ap-50 pg-100 8obj 2Man & 1.603 & 2.537 & 1.285 & 0.418 & 100 & 1.662 & 0.332 & 0.640 & 1.858 & 2.929 & 100 & 2.545 & 0.025 & 0.046 & 13.446 & 8.262 & 100 & 3.009 & 2.405 \\
\hline ecc ap-75 pg-100 8obj 2Man & 3.782 & 5.553 & 2.828 & 0.315 & 100 & 0.951 & 0.270 & 0.512 & 1.717 & 2.650 & 100 & 2.562 & 0.027 & 0.048 & 14.521 & 8.901 & 100 & 3.019 & 2.178 \\
\hline ecc ap100 pg0 8obj 2Man & 1.354 & 2.335 & 0.729 & 0.620 & 100 & 1.998 & 0.532 & 1.013 & 1.737 & 2.812 & 100 & 2.182 & 0.032 & 0.056 & 13.838 & 8.646 & 100 & 2.762 & 2.314 \\
\hline ecc ap0 pg-100 8obj 2Man & 1.233 & 2.074 & 1.026 & 0.549 & 100 & 2.093 & 0.456 & 0.864 & 1.809 & 2.788 & 100 & 2.284 & 0.028 & 0.050 & 13.977 & 8.566 & 100 & 2.906 & 2.428 \\
\hline
\end{tabular}




\section{Conclusion}

This research effort aims to address two investigative research questions: can a constellation of observer satellites engage in meaningful SSA and collision mitigation of massive orbital debris clusters, and how successful is the employed methodology at optimizing constellation designs. The results of the analysis contrasted with the hypothesized performance of the various constellation architectures, with maneuvering cases ultimately underperforming static cases. The methodology and metrics used for the analysis were specifically geared for the mission objectives, and intended to reward traits that improve both SSA and LDR intervention. The difference between the hypothesized results and the actual results highlights the effectiveness of the methodology at identifying the key aspects of the constellation design that provide the best environment for meaningful debris management and optimize debris remediation. While maneuvering cases and elliptical orbits were expected to provide performance improvements, the methodology identified constellations that most closely reflect the orbits of the debris objects, circular and static, as the ideal design. These much simpler orbits reduce the cost associated with deploying such a constellation, meaning larger constellations could be launched than would otherwise be possible. Were the clusters less uniformly distributed, I would expect more complex orbits to have improved performance and maneuvering constellations to be more useful. All constellations analyzed included eight observer satellites, but increasing the number of satellites would improve overall performance.

For a constellation to be effective, it must provide adequate coverage and interaction between the OBSs and RSOs. The constellation must be capable of monitoring every object within the cluster with enough quality and timeliness to predict all potential collisions accurately and have the time necessary to reliably intervene. The results show that a constellation of circular, non- 
maneuvering observer satellites placed at the ideal offset would provide highly effective coverage of the debris clusters. For an eight object constellation, each debris object would have an adequate LDR engagement opportunity every hour with a conjunction duration over eight minutes, and a close range conjunction every day for roughly one-minute. Based on the LDR performance figures noted in section III.B.2, roughly 52 eight-minute conjunctions are required to successfully nudge the SMA of a 1000kg object by $40 \mathrm{~km}$. The results suggest this would be achievable in under three days with an eight object constellation. While still capable of providing adequate and effective coverage, maneuvering cases provided inferior performance compared to the non-maneuvering cases, in addition to adding major mission constraints to fuel and time resources.

The methodology is successful in demonstrating its value as a systems engineering tool in constellation design and analysis of alternatives. The method is modular and can be modified and focused to fit various scenarios in which an evaluation of constellation coverage is required. The system additionally allows for easy manipulation of the weighing scheme as mission success criteria change. For example, if it were determined that duration gaps are more important than average conjunction duration due to initial LDR commissioning time being an issue, an engineer could recalculate scoring with TAG weighed heavier than MD, and a new constellation may be found to achieve superior performance. The results and processes explored in this research effort can be applied to various areas of ongoing research in an effort to develop the best practices and solutions to modern space situational awareness and satellite traffic management problems. 


\section{References}

[1] Johnson, N., "The Collision of Iridium 33 and Cosmos 2251: The Shape of Things to Come," $60^{\text {th }}$ International Astronautical Congress, Daejeon, Republic of Korea, 16 Oct. 2009

[2] McKnight, D., Walbert, K., Ohriner, E., Fairbanks, J., Lobo, A., "Proposed Series of Orbital Debris Remediation Activities," $7^{\text {th }}$ European Conference on Space Debris, Vol. 7, Darmstadt, Germany, June 2017 doi: 10.4172/2618-9792-C1-017

[3] Bonnal, C., McKnight. D., Phipps, C., Dupont, C., Missonnier, S., Lequette, L., Merle, M., Rommelaere, S., "Just in time collision avoidance - A review," Acta Astronautica, Vol. 170, May 2020, pp. 637-651 doi: 10.1016/j.actaastro.2020.02.016

[4] Phipps, C., "LADROIT- A Spaceborne Ultraviolet Laser System for Space Debris Clearing," Acta Astronautica, Vol. 104, Nov 2014, pp. 243-255

doi: 10.1016/j.actaastro.2014.08.0057

[5] Yates, J., Spanbauer, B., Black, J.T., "Geostationary Orbit Development and Evaluation for Space Situational Awareness," Acta Astronautica, Vol. 81, Iss. 1, Dec. 2012, pp. 256-272

doi: 10.1016/j.actaastro.2012.05.011

[6] McKnight, D., Rosenblatt, J., Garber, D., "Responsible Behavior for Constellations and Clusters," Space Traffic Management Conference. 8. 2018

[7] Phipps, C., Bonnal, C., "A Spaceborne, Pulsed UV Laser System for Re-entering or Nudging LEO Debris, and Re-orbiting GEO Debris," Acta Astronautica, Vol. 118, Jan 2016, pp. 224-236

doi: 10.1016/j.actaastro.2015.10.005

[8] Bekey, I., "Project ORION: Orbital Debris Removal Using Ground-Based Sensors and Lasers", $2^{\text {nd }}$ European Conference on Space Debris, ESA-SP 393, Vol. 2, 17-19 Mar. 1997, pp.699-701

[9] Cambell, J., Taylor, C., "Project ORION: results of a feasibility study on removing orbital debris using ground-based lasers and sensors", Proc. SPIE, Vol. 3126, 17 Oct. 1997

doi: $10.1117 / 12.290176$

[10] Soulard, R., Quinn, M.,"ICAN: A Novel Laser Architecture for Space Debris Removal," Acta Astronautica, Vol. 105, Dec. 2014, pp.192-200

doi: 10.1016/j.actaastro.2014.09.004

[11] Wertz, J. R., Larson, W. J., Space Mission Analysis and Design, $3^{\text {rd }}$ ed., Microcosm Press, Kluwer Academic Publishers, 1999, pp.159-202

[12] Vallado, D. A., Fundamentals of Astrodynamics and Applications, $4^{\text {th }}$ ed., Mar. 2013, pp.345-350 


\section{Appendix A: Cluster Information}

\section{$\underline{\text { Cluster 1 }}$}

\begin{tabular}{|c|c|c|c|c|c|c|c|}
\hline SAT\# & Name & Intl Desig. & Period & Inclination & Apogee & Perigee & Mass (kg) \\
\hline \multicolumn{8}{|c|}{ SL-16 Rocket Bodies } \\
\hline 16182 & SL-16 R/B & 1985-097B & 101.68 & 71 & 845 & 832 & 8,300 \\
\hline 17590 & SL-16 R/B & 1987-027B & 101.64 & 71 & 841 & 832 & 8,300 \\
\hline 17974 & SL-16 R/B & 1987-041B & 101.61 & 71.01 & 846 & 823 & 8,300 \\
\hline 19120 & SL-16 R/B & 1988-039B & 101.47 & 71.02 & 842 & 814 & 8,300 \\
\hline 19650 & SL-16 R/B & 1988-102B & 101.71 & 71 & 849 & 831 & 8,300 \\
\hline 20625 & SL-16 R/B & 1990-046B & 101.8 & 71 & 855 & 833 & 8,300 \\
\hline 22220 & SL-16 R/B & 1992-076B & 101.67 & 71 & 846 & 829 & 8,300 \\
\hline 22285 & SL-16 R/B & 1992-093B & 101.77 & 71.02 & 845 & 839 & 8,300 \\
\hline 22566 & SL-16 R/B & 1993-016B & 101.77 & 71.01 & 850 & 835 & 8,300 \\
\hline 22803 & SL-16 R/B & 1993-059B & 101.64 & 70.99 & 850 & 822 & 8,300 \\
\hline 23088 & SL-16 R/B & 1994-023B & 101.79 & 71 & 847 & 840 & 8,300 \\
\hline 23405 & SL-16 R/B & 1994-077B & 101.75 & 70.98 & 845 & 839 & 8,300 \\
\hline 23705 & SL-16 R/B & 1995-058B & 101.76 & 71.02 & 851 & 833 & 8,300 \\
\hline 24298 & SL-16 R/B & 1996-051B & 101.95 & 70.83 & 860 & 842 & 8,300 \\
\hline 25407 & SL-16 R/B & 1998-045B & 101.71 & 71.01 & 844 & 836 & 8,300 \\
\hline 26070 & SL-16 R/B & 2000-006B & 101.74 & 71 & 854 & 828 & 8,300 \\
\hline 28353 & SL-16 R/B & 2004-021B & 101.83 & 71 & 847 & 843 & 8,300 \\
\hline 31793 & SL-16 R/B & 2007-029B & 101.81 & 70.97 & 846 & 843 & 8,300 \\
\hline \multicolumn{8}{|c|}{ SL-16 Payloads } \\
\hline 16181 & COSMOS 1697 & 1985-097A & 101.88 & 70.96 & 860 & 836 & 3,250 \\
\hline 17589 & COSMOS 1833 & 1987-027A & 101.92 & 70.92 & 866 & 833 & 3,250 \\
\hline 17973 & COSMOS 1844 & 1987-041A & 101.84 & 70.9 & 868 & 823 & 3,250 \\
\hline 19119 & COSMOS 1943 & 1988-039A & 101.76 & 71 & 852 & 832 & 3,250 \\
\hline 19649 & COSMOS 1980 & 1988-102A & 101.79 & 71 & 848 & 839 & 3,250 \\
\hline 20624 & COSMOS 2082 & 1990-046A & 101.82 & 71.04 & 858 & 832 & 3,250 \\
\hline 22219 & COSMOS 2219 & 1992-076A & 101.87 & 71.06 & 861 & 833 & 3,250 \\
\hline
\end{tabular}




\begin{tabular}{|c|c|c|c|c|c|c|c|}
\hline 22284 & COSMOS 2227 & 1992-093A & 101.89 & 70.98 & 861 & 835 & 3,250 \\
\hline 22565 & COSMOS 2237 & 1993-016A & 101.95 & 70.84 & 857 & 846 & 3,250 \\
\hline 22802 & COSMOS 2263 & 1993-059A & 101.9 & 70.93 & 864 & 834 & 3,250 \\
\hline 23087 & COSMOS 2278 & 1994-023A & 101.86 & 71.05 & 852 & 842 & 3,250 \\
\hline 23404 & COSMOS 2297 & 1994-077A & 101.88 & 71.02 & 858 & 837 & 3,250 \\
\hline 23704 & COSMOS 2322 & 1995-058A & 101.89 & 70.98 & 856 & 839 & 3,250 \\
\hline 24297 & COSMOS 2333 & 1996-051A & 101.89 & 70.9 & 865 & 832 & 3,250 \\
\hline 25406 & COSMOS 2360 & $1998-045 \mathrm{~A}$ & 101.95 & 70.85 & 855 & 847 & 3,250 \\
\hline 26069 & COSMOS 2369 & 2000-006A & 101.89 & 71 & 855 & 841 & 3,250 \\
\hline 28352 & COSMOS 2406 & 2004-021A & 102.02 & 71 & 865 & 844 & 3,250 \\
\hline 31792 & COSMOS 2428 & 2007-029A & 101.96 & 70.94 & 856 & 846 & 3,250 \\
\hline \multicolumn{8}{|c|}{ Other Payloads } \\
\hline 8519 & METEOR 1-23 & $1975-124 \mathrm{~A}$ & 102.07 & 81.24 & 866 & 848 & 1200 \\
\hline 7574 & METEOR 1-20 & 1974-099A & 102.03 & 81.23 & 866 & 843 & 1200 \\
\hline 4419 & METEOR 1-5 & 1970-047A & 101.74 & 81.22 & 870 & 812 & 1200 \\
\hline 11288 & METEOR 2-4 & 1979-021A & 102 & 81.21 & 870 & 837 & 2750 \\
\hline 5731 & METEOR 1-10 & 1971-120A & 102.02 & 81.26 & 872 & 837 & 2300 \\
\hline 10514 & METEOR 2-3 & 1977-117A & 102.1 & 81.21 & 874 & 842 & 2750 \\
\hline 7209 & METEOR 1-16 & 1974-011A & 101.85 & 81.23 & 875 & 817 & 1200 \\
\hline 8026 & METEOR 2-1 & $1975-064 \mathrm{~A}$ & 102.11 & 81.28 & 875 & 842 & 2750 \\
\hline 9481 & METEOR 1-26 & 1976-102A & 102.15 & 81.22 & 877 & 843 & 1200 \\
\hline 7274 & METEOR 1-17 & 1974-025A & 102.25 & 81.23 & 877 & 853 & 1200 \\
\hline 11605 & METEOR 2-5 & 1979-095A & 102.34 & 81.21 & 878 & 861 & 2750 \\
\hline 6659 & METEOR 1-15 & 1973-034A & 102.14 & 81.19 & 879 & 841 & 1200 \\
\hline 8799 & METEOR 1-24 & 1976-032A & 102.02 & 81.26 & 879 & 830 & 1200 \\
\hline 6392 & METEOR 1-14 & 1973-015A & 102.31 & 81.25 & 879 & 856 & 1200 \\
\hline 13718 & METEOR 2-9 & 1982-116A & 101.74 & 81.25 & 880 & 802 & 2750 \\
\hline 7490 & METEOR 1-19 & 1974-083A & 102.13 & 81.19 & 882 & 837 & 1200 \\
\hline 8845 & METEOR 1-25 & 1976-043A & 102.03 & 81.26 & 882 & 827 & 1200 \\
\hline 6256 & METEOR 1-13 & $1972-085 \mathrm{~A}$ & 102.21 & 81.27 & 884 & 843 & 1200 \\
\hline 11962 & METEOR 2-6 & 1980-073A & 102.09 & 81.22 & 884 & 831 & 2750 \\
\hline 5917 & METEOR 1-11 & $1972-022 \mathrm{~A}$ & 102.23 & 81.22 & 886 & 843 & 1200 \\
\hline
\end{tabular}




\begin{tabular}{|c|c|c|c|c|c|c|c|}
\hline 7714 & METEOR 1-21 & $1975-023 \mathrm{~A}$ & 102.28 & 81.21 & 888 & 845 & 1200 \\
\hline 9661 & METEOR 2-2 & $1977-002 \mathrm{~A}$ & 102.6 & 81.27 & 889 & 875 & 2750 \\
\hline 6079 & METEOR 1-12 & $1972-049 \mathrm{~A}$ & 102.63 & 81.23 & 891 & 876 & 1200 \\
\hline 9903 & METEOR 1-27 & $1977-024 \mathrm{~A}$ & 102.25 & 81.26 & 892 & 839 & 1200 \\
\hline 12456 & METEOR 2-7 & $1981-043 \mathrm{~A}$ & 102.11 & 81.27 & 893 & 824 & 2750 \\
\hline 11165 & COSMOS 1066 & $1978-121 \mathrm{~A}$ & 102.01 & 81.24 & 889 & 819 & 2750 \\
\hline 15334 & SL-12 R/B(2) & $1984-106 \mathrm{~B}$ & 101.77 & 71 & 844 & 841 & 2458 \\
\hline 15772 & SL-12 R/B(2) & $1985-042 \mathrm{D}$ & 101.32 & 71.11 & 847 & 795 & 2458 \\
\hline 32958 & FENGYUN 3A & 2008-026A & 101.44 & 98.45 & 834 & 819 & 2295 \\
\hline 20322 & COBE & $1989-089 \mathrm{~A}$ & 102.51 & 98.97 & 884 & 870 & 2260 \\
\hline 37214 & FENGYUN 3B & 2010-059A & 101.71 & 98.85 & 852 & 827 & 2234 \\
\hline 22823 & SPOT 3 & $1993-061 \mathrm{~A}$ & 101.47 & 98.9 & 841 & 815 & 1907 \\
\hline 37849 & NPP & 2011-061A & 101.44 & 98.72 & 828 & 826 & 1842 \\
\hline 15427 & NOAA 9 & $1984-123 \mathrm{~A}$ & 101.7 & 98.76 & 850 & 829 & 1712 \\
\hline 19531 & NOAA 11 & $1988-089 \mathrm{~A}$ & 101.75 & 98.6 & 849 & 834 & 1712 \\
\hline 28654 & NOAA 18 & 2005-018A & 101.96 & 99.2 & 861 & 842 & 1479 \\
\hline 11166 & SL-3 R/B & $1978-121 \mathrm{~B}$ & 101.86 & 81.24 & 895 & 798 & 1440 \\
\hline 13719 & SL-3 R/B & $1982-116 \mathrm{~B}$ & 101.8 & 81.26 & 895 & 792 & 1440 \\
\hline 11289 & SL-3 R/B & $1979-021 \mathrm{~B}$ & 102.02 & 81.25 & 910 & 799 & 1440 \\
\hline
\end{tabular}

\section{Cluster 2}

\begin{tabular}{|c|c|c|c|c|c|c|c|}
\hline SAT\# & Name & Intl Desig & Period & Inclination & Apogee & Perigee & Mass (kg) \\
\hline \multicolumn{7}{|c|}{ SL-8 Rocket Bodies } \\
\hline 5707 & SL-8 R/B & $1971-114 \mathrm{~B}$ & 100.09 & 74.03 & 781 & 745 & 1,434 \\
\hline 6061 & SL-8 R/B & $1972-043 \mathrm{~B}$ & 99.97 & 74.06 & 771 & 743 & 1,434 \\
\hline 6324 & SL-8 R/B & $1972-104 \mathrm{~B}$ & 99.77 & 74.08 & 755 & 740 & 1,434 \\
\hline 6966 & SL-8 R/B & $1973-098 \mathrm{~B}$ & 99.8 & 74.05 & 765 & 733 & 1,434 \\
\hline 7434 & SL-8 R/B & $1974-071 \mathrm{~B}$ & 100.24 & 74.04 & 785 & 755 & 1,434 \\
\hline 8344 & SL-8 R/B & $1975-094 \mathrm{~B}$ & 100.07 & 74.06 & 776 & 747 & 1,434 \\
\hline 8459 & SL-8 R/B & $1975-112 \mathrm{~B}$ & 100.23 & 74.06 & 781 & 758 & 1,434 \\
\hline 8924 & SL-8 R/B & $1976-061 \mathrm{~B}$ & 100.21 & 74.06 & 778 & 759 & 1,434 \\
\hline
\end{tabular}




\begin{tabular}{|c|c|c|c|c|c|c|c|}
\hline 9023 & SL-8 R/B & 1976-069B & 100.04 & 74.04 & 773 & 747 & 1,434 \\
\hline 9444 & SL-8 R/B & 1976-098B & 100.15 & 74.05 & 775 & 757 & 1,434 \\
\hline 10121 & SL-8 R/B & 1977-059B & 100.3 & 74.05 & 786 & 759 & 1,434 \\
\hline 10521 & SL-8 R/B & 1977-119B & 99.96 & 74.03 & 772 & 741 & 1,434 \\
\hline 10677 & SL-8 R/B & 1978-019B & 100 & 74.04 & 769 & 748 & 1,434 \\
\hline 10962 & SL-8 R/B & 1978-063B & 99.93 & 74.08 & 773 & 737 & 1,434 \\
\hline 11112 & SL-8 R/B & 1978-105B & 100.2 & 74.03 & 792 & 744 & 1,434 \\
\hline 11427 & SL-8 R/B & 1979-060B & 100.19 & 74.02 & 782 & 753 & 1,434 \\
\hline 11511 & SL-8 R/B & 1979-078B & 100.23 & 74.04 & 781 & 758 & 1,434 \\
\hline 11574 & SL-8 R/B & 1979-089B & 99.96 & 74.07 & 768 & 745 & 1,434 \\
\hline 11870 & SL-8 R/B & 1980-056B & 100.22 & 74.05 & 781 & 757 & 1,434 \\
\hline 12443 & SL-8 R/B & 1981-041B & 100.37 & 74.05 & 780 & 772 & 1,434 \\
\hline 12792 & SL-8 R/B & 1981-084B & 100.18 & 74.03 & 777 & 757 & 1,434 \\
\hline 13028 & SL-8 R/B & 1982-001B & 100.12 & 74.04 & 778 & 750 & 1,434 \\
\hline 13149 & SL-8 R/B & 1982-037B & 100.34 & 74.04 & 783 & 766 & 1,434 \\
\hline 13242 & SL-8 R/B & 1982-051B & 100.29 & 74.04 & 791 & 754 & 1,434 \\
\hline 13649 & SL-8 R/B & 1982-109B & 100.21 & 74 & 782 & 755 & 1,434 \\
\hline 13992 & SL-8 R/B & 1983-031B & 100.28 & 74.05 & 775 & 768 & 1,434 \\
\hline 14241 & SL-8 R/B & 1983-079B & 100.27 & 74.06 & 784 & 759 & 1,434 \\
\hline 14402 & SL-8 R/B & 1983-103B & 100.29 & 74.05 & 791 & 754 & 1,434 \\
\hline 14760 & SL-8 R/B & 1984-019B & 100.29 & 74.04 & 792 & 753 & 1,434 \\
\hline 15032 & SL-8 R/B & 1984-056B & 100.37 & 74.07 & 789 & 763 & 1,434 \\
\hline 15483 & SL-8 R/B & 1985-006B & 100.27 & 74.05 & 785 & 758 & 1,434 \\
\hline 16012 & SL-8 R/B & 1985-079B & 100.27 & 74.06 & 781 & 761 & 1,434 \\
\hline 16682 & SL-8 R/B & 1986-030B & 100.3 & 74.02 & 784 & 761 & 1,434 \\
\hline 16864 & SL-8 R/B & 1986-052B & 99.97 & 74.03 & 781 & 733 & 1,434 \\
\hline 16953 & SL-8 R/B & 1986-070B & 100.19 & 74.01 & 776 & 759 & 1,434 \\
\hline 17304 & SL-8 R/B & 1987-006B & 100.17 & 74.06 & 788 & 745 & 1,434 \\
\hline 18096 & SL-8 R/B & 1987-049B & 100.25 & 74.04 & 785 & 756 & 1,434 \\
\hline 18586 & SL-8 R/B & 1987-098B & 100.23 & 74.01 & 784 & 755 & 1,434 \\
\hline 19039 & SL-8 R/B & 1988-029B & 100.12 & 74.05 & 781 & 747 & 1,434 \\
\hline
\end{tabular}




\begin{tabular}{|c|c|c|c|c|c|c|c|}
\hline 19257 & SL-8 R/B & 1988-053B & 100.19 & 74.05 & 782 & 753 & 1,434 \\
\hline 19770 & SL-8 R/B & 1989-005B & 100.09 & 74.05 & 766 & 759 & 1,434 \\
\hline 21015 & SL-8 R/B & 1990-111B & 100.29 & 74.05 & 787 & 757 & 1,434 \\
\hline 22676 & SL-8 R/B & 1993-036B & 100.43 & 74.04 & 793 & 764 & 1,434 \\
\hline 23432 & SL-8 R/B & 1994-083B & 100.46 & 74.03 & 784 & 776 & 1,434 \\
\hline \multicolumn{8}{|c|}{ SL-8 Payloads } \\
\hline 5705 & cosmos 468 & $1971-114 \mathrm{~A}$ & 100.31 & 74.02 & 785 & 762 & 750 \\
\hline 6059 & cosmos 494 & 1972-043A & 100.31 & 74.06 & 782 & 765 & 750 \\
\hline 6323 & cosmos 540 & $1972-104 \mathrm{~A}$ & 100.26 & 74.08 & 783 & 758 & 750 \\
\hline 6965 & cosmos 614 & 1973-098A & 100.08 & 74.06 & 778 & 746 & 750 \\
\hline 7433 & cosmos 676 & $1974-071 \mathrm{~A}$ & 100.53 & 74.05 & 794 & 773 & 750 \\
\hline 8343 & cosmos 773 & $1975-094 \mathrm{~A}$ & 100.36 & 74.05 & 784 & 767 & 750 \\
\hline 8458 & cosmos 783 & $1975-112 A$ & 100.51 & 74.06 & 791 & 775 & 750 \\
\hline 8923 & cosmos 836 & $1976-061 \mathrm{~A}$ & 100.49 & 74.05 & 795 & 768 & 750 \\
\hline 9022 & cosmos 841 & 1976-069A & 100.3 & 74.04 & 781 & 764 & 750 \\
\hline 9443 & cosmos 858 & 1976-098A & 100.42 & 74.05 & 789 & 768 & 750 \\
\hline 10120 & cosmos 923 & 1977-059A & 100.57 & 74.05 & 796 & 775 & 750 \\
\hline 10520 & cosmos 968 & $1977-119 \mathrm{~A}$ & 100.28 & 74.03 & 784 & 760 & 750 \\
\hline 10676 & cosmos 990 & 1978-019A & 100.27 & 74.04 & 783 & 760 & 750 \\
\hline 10961 & cosmos 1023 & 1978-063A & 100.25 & 74.08 & 783 & 758 & 750 \\
\hline 11111 & COSMOS 1048 & $1978-105 A$ & 100.41 & 74.03 & 789 & 767 & 750 \\
\hline 11425 & cosmos 1110 & 1979-060A & 100.48 & 74.02 & 792 & 770 & 750 \\
\hline 11510 & cosmos 1125 & 1979-078A & 100.48 & 74.04 & 790 & 773 & 750 \\
\hline 11573 & cosmos 1140 & 1979-089A & 100.24 & 74.07 & 782 & 758 & 750 \\
\hline 11869 & COSMOS 1190 & $1980-056 \mathrm{~A}$ & 100.45 & 74.05 & 791 & 769 & 750 \\
\hline 12442 & cosmos 1269 & 1981-041A & 100.55 & 74.05 & 792 & 777 & 750 \\
\hline 12791 & COSMOS 1302 & 1981-084A & 100.42 & 74.03 & 791 & 766 & 750 \\
\hline 13027 & cosmos 1331 & $1982-001 \mathrm{~A}$ & 100.24 & 74.05 & 787 & 753 & 750 \\
\hline 13148 & COSMOS 1354 & 1982-037A & 100.57 & 74.04 & 793 & 778 & 750 \\
\hline 13241 & COSMOS 1371 & $1982-051 \mathrm{~A}$ & 100.59 & 74.04 & 796 & 777 & 750 \\
\hline 13648 & cosmos 1420 & $1982-109 A$ & 100.44 & 74 & 794 & 764 & 750 \\
\hline
\end{tabular}




\begin{tabular}{|l|l|l|l|l|l|l|l|}
\hline 13991 & COSMOS 1452 & $1983-031 \mathrm{~A}$ & 100.49 & 74.05 & 794 & 770 & 750 \\
\hline 14240 & COSMOS 1486 & $1983-079 \mathrm{~A}$ & 100.43 & 74.06 & 789 & 769 & 750 \\
\hline 14401 & COSMOS 1503 & $1983-103 \mathrm{~A}$ & 100.55 & 74.05 & 795 & 775 & 750 \\
\hline 14759 & COSMOS 1538 & $1984-019 \mathrm{~A}$ & 100.45 & 74.04 & 794 & 766 & 750 \\
\hline 15031 & COSMOS 1570 & $1984-056 \mathrm{~A}$ & 100.58 & 74.07 & 795 & 777 & 750 \\
\hline 15482 & COSMOS 1624 & $1985-006 \mathrm{~A}$ & 100.49 & 74.04 & 792 & 772 & 750 \\
\hline 16011 & COSMOS 1680 & $1985-079 \mathrm{~A}$ & 100.48 & 74.05 & 792 & 771 & 750 \\
\hline 16681 & COSMOS 1741 & $1986-030 \mathrm{~A}$ & 100.5 & 74.02 & 796 & 768 & 750 \\
\hline 16860 & COSMOS 1763 & $1986-052 \mathrm{~A}$ & 100.13 & 74.03 & 788 & 742 & 750 \\
\hline 16952 & COSMOS 1777 & $1986-070 \mathrm{~A}$ & 100.49 & 74.01 & 801 & 762 & 750 \\
\hline 17303 & COSMOS 1814 & $1987-006 \mathrm{~A}$ & 100.36 & 74.06 & 793 & 758 & 750 \\
\hline 18095 & COSMOS 1850 & $1987-049 \mathrm{~A}$ & 100.48 & 74.04 & 791 & 771 & 750 \\
\hline 18585 & COSMOS 1898 & $1987-098 \mathrm{~A}$ & 100.45 & 74.01 & 794 & 766 & 750 \\
\hline 19038 & COSMOS 1937 & $1988-029 \mathrm{~A}$ & 100.34 & 74.05 & 792 & 757 & 750 \\
\hline 19256 & COSMOS 1954 & $1988-053 \mathrm{~A}$ & 100.43 & 74.05 & 790 & 768 & 750 \\
\hline 19769 & COSMOS 1992 & $1989-005 \mathrm{~A}$ & 100.37 & 74.05 & 791 & 760 & 750 \\
\hline 21014 & COSMOS 2112 & $1990-111 \mathrm{~A}$ & 100.47 & 74.05 & 800 & 761 & 750 \\
\hline 22675 & COSMOS 2251 & $1993-036 \mathrm{~A}$ & 100.53 & 74.04 & 801 & 766 & 750 \\
\hline 23431 & COSMOS 2298 & $1994-083 \mathrm{~A}$ & 100.68 & 74.03 & 802 & 780 & 750 \\
\hline
\end{tabular}

\section{Cluster 3}

\begin{tabular}{|c|c|c|c|c|c|c|c|}
\hline Sat\# & Name & Intl Desig & Period & Inclination & Apogee & Perigee & Mass (kg) \\
\hline \multicolumn{7}{|c|}{ SL-8 Rocket Bodies } \\
\hline 4784 & SL-8 R/B & $1970-102 B$ & 104.6 & 74.03 & 995 & 957 & 1,434 \\
\hline 4800 & SL-8 R/B & $1970-108 B$ & 104.45 & 74.02 & 975 & 962 & 1,434 \\
\hline 5239 & SL-8 R/B & $1971-046 \mathrm{~B}$ & 104.82 & 74.03 & 993 & 978 & 1,434 \\
\hline 5685 & SL-8 R/B & $1971-111 B$ & 104.59 & 74.03 & 993 & 958 & 1,434 \\
\hline 5847 & SL-8 R/B & $1972-009 B$ & 104.41 & 74.05 & 989 & 944 & 1,434 \\
\hline 6020 & SL-8 R/B & $1972-035 B$ & 104.44 & 74.02 & 984 & 952 & 1,434 \\
\hline
\end{tabular}




\begin{tabular}{|c|c|c|c|c|c|c|c|}
\hline 6149 & SL-8 R/B & 1972-062B & 104.13 & 82.97 & 960 & 948 & 1,434 \\
\hline 6207 & SL-8 R/B & 1972-074B & 104.68 & 65.82 & 1002 & 957 & 1,434 \\
\hline 6708 & SL-8 R/B & 1973-042B & 104.8 & 82.95 & 994 & 976 & 1,434 \\
\hline 6829 & SL-8 R/B & 1973-065B & 104.57 & 82.94 & 990 & 958 & 1,434 \\
\hline 7009 & SL-8 R/B & 1973-109B & 104.59 & 82.95 & 989 & 962 & 1,434 \\
\hline 7095 & SL-8 R/B & 1974-001B & 104.46 & 82.95 & 997 & 941 & 1,434 \\
\hline 7350 & SL-8 R/B & 1974-048B & 104.52 & 82.94 & 984 & 960 & 1,434 \\
\hline 7477 & SL-8 R/B & 1974-079B & 104.8 & 82.94 & 1011 & 959 & 1,434 \\
\hline 7594 & SL-8 R/B & 1974-105B & 104.44 & 82.95 & 980 & 956 & 1,434 \\
\hline 7737 & SL-8 R/B & 1975-028B & 104.32 & 83 & 976 & 949 & 1,434 \\
\hline 7769 & SL-8 R/B & 1975-034B & 104.74 & 82.96 & 996 & 969 & 1,434 \\
\hline 8073 & SL-8 R/B & $1975-074 \mathrm{~B}$ & 104.66 & 82.9 & 995 & 962 & 1,434 \\
\hline 8421 & SL-8 R/B & 1975-103B & 104.6 & 82.97 & 991 & 960 & 1,434 \\
\hline 8597 & SL-8 R/B & 1976-005B & 104.72 & 82.97 & 999 & 964 & 1,434 \\
\hline 8646 & SL-8 R/B & 1976-011B & 104.82 & 82.97 & 989 & 983 & 1,434 \\
\hline 8874 & SL-8 R/B & 1976-051B & 104.74 & 82.96 & 999 & 965 & 1,434 \\
\hline 9044 & SL-8 R/B & 1976-070B & 104.64 & 82.99 & 987 & 968 & 1,434 \\
\hline 9062 & SL-8 R/B & 1976-078B & 104.47 & 82.93 & 990 & 949 & 1,434 \\
\hline 9510 & SL-8 R/B & 1976-108B & 104.54 & 82.93 & 992 & 954 & 1,434 \\
\hline 9613 & SL-8 R/B & 1976-122B & 104.55 & 82.95 & 994 & 952 & 1,434 \\
\hline 9638 & SL-8 R/B & 1976-128B & 104.48 & 82.94 & 993 & 948 & 1,434 \\
\hline 9738 & SL-8 R/B & 1977-004B & 104.83 & 82.96 & 998 & 975 & 1,434 \\
\hline 9848 & SL-8 R/B & 1977-013B & 104.69 & 82.95 & 991 & 969 & 1,434 \\
\hline 10020 & SL-8 R/B & 1977-039B & 104.5 & 82.94 & 993 & 949 & 1,434 \\
\hline 10138 & SL-8 R/B & 1977-062B & 104.82 & 82.94 & 1002 & 970 & 1,434 \\
\hline 10142 & SL-8 R/B & 1977-064B & 104.45 & 82.96 & 1001 & 937 & 1,434 \\
\hline 10355 & SL-8 R/B & 1977-087B & 104.67 & 82.97 & 1005 & 953 & 1,434 \\
\hline 10461 & SL-8 R/B & 1977-107B & 104.59 & 82.95 & 1000 & 950 & 1,434 \\
\hline 10513 & SL-8 R/B & 1977-116B & 104.46 & 65.83 & 995 & 943 & 1,434 \\
\hline 10537 & SL-8 R/B & $1977-122 \mathrm{~B}$ & 104.71 & 82.93 & 996 & 966 & 1,434 \\
\hline 10600 & SL-8 R/B & 1978-007B & 104.47 & 82.94 & 1004 & 935 & 1,434 \\
\hline
\end{tabular}




\begin{tabular}{|c|c|c|c|c|c|c|c|}
\hline 10693 & SL-8 R/B & 1978-022B & 104.54 & 82.99 & 990 & 956 & 1,434 \\
\hline 10732 & SL-8 R/B & 1978-028B & 104.7 & 82.93 & 994 & 967 & 1,434 \\
\hline 10745 & SL-8 R/B & 1978-031B & 104.46 & 82.93 & 996 & 942 & 1,434 \\
\hline 10777 & SL-8 R/B & 1978-034B & 104.55 & 82.94 & 992 & 954 & 1,434 \\
\hline 10918 & SL-8 R/B & 1978-053B & 104.55 & 82.92 & 996 & 951 & 1,434 \\
\hline 10992 & SL-8 R/B & 1978-074B & 104.51 & 82.93 & 987 & 957 & 1,434 \\
\hline 11239 & SL-8 R/B & 1979-003B & 104.67 & 82.94 & 1010 & 948 & 1,434 \\
\hline 11309 & SL-8 R/B & 1979-026B & 104.57 & 82.97 & 989 & 959 & 1,434 \\
\hline 11321 & SL-8 R/B & 1979-028B & 104.62 & 82.92 & 990 & 963 & 1,434 \\
\hline 11327 & SL-8 R/B & 1979-030B & 104.58 & 82.95 & 995 & 954 & 1,434 \\
\hline 11379 & SL-8 R/B & 1979-046B & 104.55 & 82.95 & 988 & 958 & 1,434 \\
\hline 11586 & SL-8 R/B & 1979-090B & 104.43 & 82.94 & 987 & 948 & 1,434 \\
\hline 11668 & SL-8 R/B & 1980-003B & 104.68 & 82.95 & 995 & 964 & 1,434 \\
\hline 11681 & SL-8 R/B & 1980-007B & 104.7 & 82.93 & 1007 & 954 & 1,434 \\
\hline 11736 & SL-8 R/B & 1980-022B & 104.62 & 82.94 & 1002 & 951 & 1,434 \\
\hline 11751 & SL-8 R/B & 1980-026B & 104.57 & 65.84 & 987 & 962 & 1,434 \\
\hline 11804 & SL-8 R/B & 1980-039B & 104.64 & 82.94 & 992 & 963 & 1,434 \\
\hline 12088 & SL-8 R/B & 1980-097B & 104.58 & 82.94 & 1012 & 938 & 1,434 \\
\hline 12092 & SL-8 R/B & 1980-099B & 104.58 & 82.94 & 998 & 952 & 1,434 \\
\hline 12298 & SL-8 R/B & 1981-013B & 104.61 & 82.95 & 997 & 955 & 1,434 \\
\hline 12508 & SL-8 R/B & 1981-053B & 104.63 & 82.96 & 1004 & 950 & 1,434 \\
\hline 12682 & SL-8 R/B & 1981-077B & 104.46 & 82.92 & 994 & 944 & 1,434 \\
\hline 12836 & SL-8 R/B & 1981-091B & 104.62 & 82.92 & 993 & 960 & 1,434 \\
\hline 13034 & SL-8 R/B & 1982-003B & 104.7 & 82.94 & 1004 & 957 & 1,434 \\
\hline 13066 & SL-8 R/B & 1982-012B & 104.56 & 82.9 & 1005 & 943 & 1,434 \\
\hline 13111 & SL-8 R/B & 1982-024B & 104.67 & 82.93 & 1010 & 948 & 1,434 \\
\hline 13128 & SL-8 R/B & 1982-030B & 104.67 & 82.92 & 1001 & 957 & 1,434 \\
\hline 13260 & SL-8 R/B & 1982-055B & 104.78 & 65.83 & 1018 & 950 & 1,434 \\
\hline 13618 & SL-8 R/B & 1982-102B & 104.6 & 82.97 & 1000 & 951 & 1,434 \\
\hline 13758 & SL-8 R/B & 1983-001B & 104.45 & 82.91 & 988 & 949 & 1,434 \\
\hline 13917 & SL-8 R/B & 1983-021B & 104.59 & 82.94 & 995 & 955 & 1,434 \\
\hline
\end{tabular}




\begin{tabular}{|c|c|c|c|c|c|c|c|}
\hline 13950 & SL-8 R/B & 1983-023B & 104.59 & 82.96 & 1001 & 949 & 1,434 \\
\hline 14059 & SL-8 R/B & 1983-042B & 104.46 & 82.97 & 999 & 939 & 1,434 \\
\hline 14085 & SL-8 R/B & 1983-048B & 104.63 & 82.95 & 1003 & 952 & 1,434 \\
\hline 14451 & SL-8 R/B & 1983-108B & 104.5 & 82.93 & 996 & 946 & 1,434 \\
\hline 14547 & SL-8 R/B & 1983-120B & 104.57 & 82.93 & 1008 & 941 & 1,434 \\
\hline 14625 & SL-8 R/B & 1984-003B & 104.78 & 82.93 & 1001 & 967 & 1,434 \\
\hline 14680 & SL-8 R/B & 1984-010B & 104.61 & 82.95 & 1005 & 948 & 1,434 \\
\hline 14966 & SL-8 R/B & 1984-043B & 104.77 & 82.97 & 996 & 971 & 1,434 \\
\hline 14974 & SL-8 R/B & 1984-046B & 104.58 & 82.94 & 1008 & 941 & 1,434 \\
\hline 15056 & SL-8 R/B & 1984-062B & 104.63 & 82.96 & 997 & 958 & 1,434 \\
\hline 15078 & SL-8 R/B & 1984-067B & 104.57 & 82.96 & 994 & 955 & 1,434 \\
\hline 15293 & SL-8 R/B & 1984-100B & 104.73 & 82.94 & 999 & 964 & 1,434 \\
\hline 15360 & SL-8 R/B & 1984-109B & 104.6 & 82.94 & 1007 & 944 & 1,434 \\
\hline 15399 & SL-8 R/B & 1984-118B & 104.65 & 82.94 & 1003 & 953 & 1,434 \\
\hline 15506 & SL-8 R/B & 1985-011B & 104.64 & 82.92 & 1005 & 950 & 1,434 \\
\hline 15598 & SL-8 R/B & 1985-022B & 104.61 & 82.94 & 993 & 959 & 1,434 \\
\hline 15752 & SL-8 R/B & 1985-041B & 104.87 & 82.95 & 1007 & 970 & 1,434 \\
\hline 16292 & SL-8 R/B & 1985-110B & 104.58 & 82.93 & 998 & 952 & 1,434 \\
\hline 16369 & SL-8 R/B & 1985-116B & 104.59 & 82.94 & 1003 & 948 & 1,434 \\
\hline 16494 & SL-8 R/B & 1986-005B & 104.62 & 82.93 & 990 & 963 & 1,434 \\
\hline 16511 & SL-8 R/B & 1986-008B & 104.67 & 82.94 & 996 & 962 & 1,434 \\
\hline 16728 & SL-8 R/B & 1986-037B & 104.6 & 82.96 & 998 & 953 & 1,434 \\
\hline 17067 & SL-8 R/B & 1986-086B & 104.53 & 82.95 & 997 & 947 & 1,434 \\
\hline 17160 & SL-8 R/B & 1986-093B & 104.76 & 82.93 & 1012 & 954 & 1,434 \\
\hline 17240 & SL-8 R/B & 1986-100B & 104.81 & 82.93 & 1007 & 964 & 1,434 \\
\hline 17360 & SL-8 R/B & 1987-009B & 104.6 & 82.92 & 999 & 952 & 1,434 \\
\hline 17526 & SL-8 R/B & 1987-017B & 104.59 & 82.9 & 1004 & 946 & 1,434 \\
\hline 18130 & SL-8 R/B & 1987-054B & 104.61 & 82.92 & 989 & 964 & 1,434 \\
\hline 18161 & SL-8 R/B & 1987-057B & 104.58 & 82.93 & 997 & 952 & 1,434 \\
\hline 18710 & SL-8 R/B & 1987-106B & 104.65 & 82.91 & 996 & 960 & 1,434 \\
\hline 18986 & SL-8 R/B & 1988-023B & 104.45 & 82.95 & 999 & 939 & 1,434 \\
\hline
\end{tabular}




\begin{tabular}{|c|c|c|c|c|c|c|c|}
\hline 19325 & SL-8 R/B & 1988-062B & 104.54 & 82.95 & 997 & 949 & 1,434 \\
\hline 19827 & SL-8 R/B & 1989-017B & 104.8 & 82.95 & 1007 & 963 & 1,434 \\
\hline 19922 & SL-8 R/B & 1989-028B & 104.56 & 82.96 & 1000 & 948 & 1,434 \\
\hline 20046 & SL-8 R/B & 1989-042B & 104.49 & 82.94 & 1003 & 939 & 1,434 \\
\hline 20104 & SL-8 R/B & 1989-050B & 104.56 & 82.96 & 1005 & 942 & 1,434 \\
\hline 20150 & SL-8 R/B & 1989-059B & 104.68 & 82.94 & 1000 & 959 & 1,434 \\
\hline 20509 & SL-8 R/B & 1990-017B & 104.69 & 82.95 & 1012 & 948 & 1,434 \\
\hline 20528 & SL-8 R/B & 1990-023B & 104.89 & 82.93 & 1013 & 965 & 1,434 \\
\hline 20578 & SL-8 R/B & 1990-036B & 104.63 & 82.94 & 986 & 968 & 1,434 \\
\hline 20805 & SL-8 R/B & 1990-083B & 104.67 & 82.94 & 1002 & 956 & 1,434 \\
\hline 21088 & SL-8 R/B & 1991-006B & 104.57 & 82.94 & 990 & 958 & 1,434 \\
\hline 21090 & SL-8 R/B & 1991-007B & 104.63 & 82.92 & 993 & 961 & 1,434 \\
\hline 21153 & SL-8 R/B & 1991-019B & 104.66 & 82.92 & 1001 & 957 & 1,434 \\
\hline 21231 & SL-8 R/B & 1991-029B & 104.67 & 82.95 & 1004 & 954 & 1,434 \\
\hline 21667 & SL-8 R/B & 1991-059B & 104.72 & 82.9 & 996 & 967 & 1,434 \\
\hline 21797 & SL-8 R/B & 1991-081B & 104.55 & 82.95 & 1002 & 944 & 1,434 \\
\hline 21876 & SL-8 R/B & 1992-008B & 104.68 & 82.93 & 1006 & 953 & 1,434 \\
\hline 21903 & SL-8 R/B & 1992-012B & 104.77 & 82.94 & 1016 & 951 & 1,434 \\
\hline 21938 & SL-8 R/B & 1992-020B & 104.71 & 82.93 & 1002 & 960 & 1,434 \\
\hline 22007 & SL-8 R/B & 1992-036B & 104.51 & 82.93 & 993 & 950 & 1,434 \\
\hline 22208 & SL-8 R/B & 1992-073B & 104.72 & 82.92 & 1007 & 955 & 1,434 \\
\hline 22308 & SL-8 R/B & 1993-001B & 104.68 & 82.93 & 996 & 963 & 1,434 \\
\hline 22488 & SL-8 R/B & 1993-008B & 104.52 & 82.94 & 990 & 954 & 1,434 \\
\hline 22591 & SL-8 R/B & 1993-020B & 104.55 & 82.93 & 992 & 954 & 1,434 \\
\hline 22889 & SL-8 R/B & 1993-070B & 104.51 & 82.95 & 1007 & 936 & 1,434 \\
\hline 23093 & SL-8 R/B & 1994-024B & 104.52 & 82.95 & 980 & 964 & 1,434 \\
\hline 23180 & SL-8 R/B & 1994-041B & 104.5 & 82.95 & 992 & 951 & 1,434 \\
\hline 23190 & SL-8 R/B & 1994-045B & 104.8 & 74.03 & 999 & 971 & 1,434 \\
\hline 23466 & SL-8 R/B & 1995-002D & 104.78 & 82.93 & 1009 & 959 & 1,434 \\
\hline 23527 & SL-8 R/B & 1995-012B & 104.84 & 82.94 & 1001 & 973 & 1,434 \\
\hline 23604 & SL-8 R/B & 1995-032B & 104.78 & 82.9 & 1001 & 967 & 1,434 \\
\hline
\end{tabular}




\begin{tabular}{|c|c|c|c|c|c|c|c|}
\hline 23774 & SL-8 R/B & 1996-004B & 104.62 & 82.98 & 1009 & 944 & 1,434 \\
\hline 24306 & SL-8 R/B & 1996-052C & 104.73 & 82.93 & 1002 & 962 & 1,434 \\
\hline 24678 & SL-8 R/B & 1996-071B & 104.83 & 82.94 & 1004 & 969 & 1,434 \\
\hline 24773 & SL-8 R/B & 1997-017B & 104.84 & 82.92 & 1004 & 970 & 1,434 \\
\hline 24955 & SL-8 R/B & $1997-052 C$ & 104.24 & 82.92 & 981 & 936 & 1,434 \\
\hline 25569 & SL-8 R/B & $1998-072 C$ & 104.81 & 82.95 & 1002 & 969 & 1,434 \\
\hline 25592 & SL-8 R/B & 1998-076B & 104.8 & 82.94 & 1004 & 967 & 1,434 \\
\hline 25893 & SL-8 R/B & 1999-045B & 104.62 & 82.92 & 998 & 954 & 1,434 \\
\hline 26819 & SL-8 R/B & 2001-023B & 104.69 & 82.93 & 997 & 963 & 1,434 \\
\hline 27437 & SL-8 R/B & 2002-026B & 104.61 & 82.95 & 1009 & 943 & 1,434 \\
\hline 27535 & SL-8 R/B & 2002-046B & 104.78 & 82.93 & 1012 & 956 & 1,434 \\
\hline 27819 & SL-8 R/B & 2003-023B & 104.79 & 82.95 & 1020 & 949 & 1,434 \\
\hline 28381 & SL-8 R/B & 2004-028B & 104.49 & 82.96 & 994 & 946 & 1,434 \\
\hline 32053 & SL-8 R/B & 2007-038B & 104.63 & 82.98 & 998 & 957 & 1,434 \\
\hline \multicolumn{8}{|c|}{ SI-8 Payloads } \\
\hline 4783 & cosMOS 381 & $1970-102 A$ & 104.75 & 74.03 & 1005 & 961 & 700 \\
\hline 4799 & cosmos 385 & $1970-108 \mathrm{~A}$ & 104.6 & 74.02 & 978 & 973 & 700 \\
\hline 5238 & cosmos 422 & $1971-046 \mathrm{~A}$ & 104.95 & 74.02 & 1003 & 981 & 700 \\
\hline 5683 & cosmos 465 & $1971-111 \mathrm{~A}$ & 104.79 & 74.03 & 1004 & 965 & 700 \\
\hline 5846 & cosmos 475 & $1972-009 A$ & 104.64 & 74.05 & 994 & 961 & 700 \\
\hline 6019 & cosmos 489 & 1972-035A & 104.66 & 74.02 & 997 & 960 & 700 \\
\hline 6148 & cosmos 514 & $1972-062 A$ & 104.22 & 82.97 & 966 & 949 & 700 \\
\hline 6206 & cosmos 521 & $1972-074 \mathrm{~A}$ & 104.92 & 65.83 & 995 & 987 & 700 \\
\hline 6707 & cosmos 574 & $1973-042 A$ & 104.95 & 82.95 & 1008 & 976 & 700 \\
\hline 6828 & cosmos 586 & $1973-065 A$ & 104.72 & 82.94 & 1002 & 961 & 700 \\
\hline 7008 & cosmos 627 & 1973-109A & 104.89 & 82.95 & 1014 & 965 & 700 \\
\hline 7094 & cosmos 628 & $1974-001 \mathrm{~A}$ & 104.67 & 82.96 & 1006 & 953 & 700 \\
\hline 7349 & cosmos 663 & $1974-048 \mathrm{~A}$ & 104.69 & 82.95 & 999 & 960 & 700 \\
\hline 7476 & cosmos 689 & 1974-079A & 104.95 & 82.94 & 1013 & 971 & 700 \\
\hline 7593 & cosmos 700 & $1974-105 A$ & 104.6 & 82.95 & 994 & 957 & 700 \\
\hline 7736 & cosmos 726 & 1975-028A & 104.49 & 83 & 988 & 952 & 700 \\
\hline
\end{tabular}




\begin{tabular}{|c|c|c|c|c|c|c|c|}
\hline 7768 & cosmos 729 & 1975-034A & 104.85 & 82.96 & 1004 & 971 & 700 \\
\hline 8072 & cosmos 755 & 1975-074A & 104.81 & 82.9 & 1005 & 966 & 700 \\
\hline 8419 & cosmos 778 & 1975-103A & 104.76 & 82.97 & 1000 & 966 & 700 \\
\hline 8591 & cosmos 789 & $1976-005 A$ & 104.88 & 82.98 & 1011 & 967 & 700 \\
\hline 8645 & cosmos 800 & $1976-011 \mathrm{~A}$ & 104.96 & 82.97 & 1009 & 976 & 700 \\
\hline 8873 & cosmos 823 & $1976-051 \mathrm{~A}$ & 104.86 & 82.96 & 1005 & 971 & 700 \\
\hline 9025 & cosmos 842 & 1976-070A & 104.8 & 82.99 & 1007 & 964 & 700 \\
\hline 9061 & cosmos 846 & 1976-078A & 104.62 & 82.93 & 1007 & 946 & 700 \\
\hline 9509 & COSMOS 864 & 1976-108A & 104.7 & 82.94 & 1002 & 959 & 700 \\
\hline 9610 & cosmOS 883 & $1976-122 \mathrm{~A}$ & 104.66 & 82.95 & 1005 & 952 & 700 \\
\hline 9637 & cosmos 887 & $1976-128 \mathrm{~A}$ & 104.64 & 82.94 & 1012 & 943 & 700 \\
\hline 9737 & cosmos 890 & $1977-004 \mathrm{~A}$ & 104.99 & 82.96 & 1014 & 974 & 700 \\
\hline 9846 & cosmos 894 & 1977-013A & 104.81 & 82.94 & 1008 & 963 & 700 \\
\hline 10019 & COSMOS 911 & 1977-039A & 104.71 & 82.95 & 999 & 962 & 700 \\
\hline 10137 & cosmos 926 & $1977-062 A$ & 104.94 & 82.94 & 1016 & 967 & 700 \\
\hline 10141 & COSMOS 928 & 1977-064A & 104.61 & 82.97 & 1003 & 949 & 700 \\
\hline 10352 & cosmos 951 & 1977-087A & 104.8 & 82.97 & 1010 & 960 & 700 \\
\hline 10459 & cosmos 962 & 1977-107A & 104.75 & 82.95 & 1003 & 963 & 700 \\
\hline 10512 & cosmos 967 & $1977-116 \mathrm{~A}$ & 104.73 & 65.83 & 1004 & 960 & 700 \\
\hline 10536 & cosmos 971 & $1977-122 \mathrm{~A}$ & 104.87 & 82.94 & 1004 & 972 & 700 \\
\hline 10599 & cosmOS 985 & 1978-007A & 104.6 & 82.94 & 1015 & 936 & 700 \\
\hline 10692 & COSMOS 991 & $1978-022 A$ & 104.63 & 82.99 & 1005 & 950 & 700 \\
\hline 10731 & cosmos 994 & 1978-028A & 104.87 & 82.93 & 1006 & 970 & 700 \\
\hline 10744 & cosmos 996 & $1978-031 \mathrm{~A}$ & 104.62 & 82.93 & 1003 & 950 & 700 \\
\hline 10776 & cosmos 1000 & $1978-034 \mathrm{~A}$ & 104.7 & 82.94 & 1005 & 956 & 700 \\
\hline 10917 & COSMOS 1011 & 1978-053A & 104.71 & 82.92 & 1009 & 953 & 700 \\
\hline 10991 & cosmos 1027 & $1978-074 \mathrm{~A}$ & 104.62 & 82.94 & 996 & 958 & 700 \\
\hline 11238 & cosmos 1072 & 1979-003A & 104.79 & 82.94 & 1013 & 957 & 700 \\
\hline 11308 & COSMOS 1089 & 1979-026A & 104.72 & 82.97 & 997 & 965 & 700 \\
\hline 11320 & COSMOS 1091 & $1979-028 \mathrm{~A}$ & 104.76 & 82.92 & 1005 & 961 & 700 \\
\hline 11326 & COSMOS 1092 & 1979-030A & 104.7 & 82.95 & 1001 & 960 & 700 \\
\hline
\end{tabular}




\begin{tabular}{|c|c|c|c|c|c|c|c|}
\hline 11378 & COSMOS 1104 & 1979-046A & 104.67 & 82.95 & 1004 & 954 & 700 \\
\hline 11667 & cosmos 1150 & 1980-003A & 104.85 & 82.95 & 1012 & 963 & 700 \\
\hline 11680 & cosmos 1153 & 1980-007A & 104.83 & 82.93 & 1015 & 958 & 700 \\
\hline 11735 & COSMOS 1168 & $1980-022 A$ & 104.73 & 82.94 & 1008 & 956 & 700 \\
\hline 11750 & COSMOS 1171 & 1980-026A & 104.83 & 65.84 & 1009 & 964 & 700 \\
\hline 11803 & COSMOS 1181 & 1980-039A & 104.81 & 82.95 & 1003 & 968 & 700 \\
\hline 12087 & cosmos 1225 & 1980-097A & 104.77 & 82.94 & 1024 & 943 & 700 \\
\hline 12091 & cosmos 1226 & 1980-099A & 104.77 & 82.94 & 1008 & 960 & 700 \\
\hline 12297 & COSMOS 1244 & 1981-013A & 104.73 & 82.95 & 1004 & 959 & 700 \\
\hline 12504 & cosmos 1275 & 1981-053A & 104.78 & 82.96 & 1013 & 955 & 700 \\
\hline 12681 & COSMOS 1295 & 1981-077A & 104.62 & 82.92 & 1010 & 943 & 700 \\
\hline 12835 & COSMOS 1308 & 1981-091A & 104.7 & 82.92 & 1000 & 961 & 700 \\
\hline 13033 & COSMOS 1333 & 1982-003A & 104.87 & 82.94 & 1012 & 965 & 700 \\
\hline 13065 & cosmos 1339 & 1982-012A & 104.69 & 82.9 & 1011 & 949 & 700 \\
\hline 13110 & cosmos 1344 & 1982-024A & 104.81 & 82.92 & 1007 & 964 & 700 \\
\hline 13127 & cosmos 1349 & $1982-030 \mathrm{~A}$ & 104.82 & 82.93 & 1009 & 964 & 700 \\
\hline 13259 & COSMOS 1375 & 1982-055A & 104.14 & 65.83 & 974 & 934 & 600 \\
\hline 13757 & COSMOS 1428 & 1983-001A & 104.59 & 82.91 & 1002 & 949 & 810 \\
\hline 13916 & cosmos 1447 & 1983-021A & 104.72 & 82.95 & 1009 & 954 & 810 \\
\hline 13949 & cosMOS 1448 & 1983-023A & 104.66 & 82.96 & 1000 & 958 & 810 \\
\hline 14057 & cosmos 1459 & 1983-042A & 104.61 & 82.97 & 1013 & 939 & 810 \\
\hline 14084 & COSMOS 1464 & 1983-048A & 104.77 & 82.95 & 1006 & 962 & 810 \\
\hline 14450 & cosmos 1506 & 1983-108A & 104.64 & 82.93 & 1009 & 946 & 810 \\
\hline 14546 & cosmos 1513 & 1983-120A & 104.79 & 82.94 & 1014 & 955 & 810 \\
\hline 14624 & COSMOS 1531 & 1984-003A & 104.95 & 82.93 & 1006 & 978 & 810 \\
\hline 14679 & cosmos 1535 & 1984-010A & 104.72 & 82.96 & 1013 & 950 & 810 \\
\hline 14965 & COSMOS 1550 & 1984-043A & 104.9 & 82.98 & 1009 & 970 & 810 \\
\hline 14973 & cosmos 1553 & 1984-046A & 104.71 & 82.94 & 1005 & 956 & 810 \\
\hline 15055 & COSMOS 1574 & 1984-062A & 104.78 & 82.96 & 1004 & 965 & 810 \\
\hline 15077 & COSMOS 1577 & 1984-067A & 104.7 & 82.96 & 1007 & 954 & 810 \\
\hline 15292 & COSMOS 1598 & 1984-100A & 104.88 & 82.95 & 1013 & 965 & 810 \\
\hline
\end{tabular}




\begin{tabular}{|c|c|c|c|c|c|c|c|}
\hline 15359 & COSMOS 1605 & 1984-109A & 104.72 & 82.94 & 1016 & 946 & 810 \\
\hline 15398 & COSMOS 1610 & 1984-118A & 104.83 & 82.95 & 1009 & 964 & 810 \\
\hline 15505 & COSMOS 1627 & $1985-011 \mathrm{~A}$ & 104.78 & 82.92 & 1016 & 952 & 810 \\
\hline 15597 & COSMOS 1634 & $1985-022 \mathrm{~A}$ & 104.72 & 82.94 & 1007 & 956 & 2200 \\
\hline 15751 & cosmOS 1655 & $1985-041 \mathrm{~A}$ & 104.98 & 82.95 & 1013 & 974 & 810 \\
\hline 16291 & COSMOS 1704 & 1985-110A & 104.76 & 82.93 & 1006 & 961 & 810 \\
\hline 16368 & cosmos 1709 & 1985-116A & 104.77 & 82.94 & 1008 & 959 & 810 \\
\hline 16493 & cosmos 1725 & 1986-005A & 104.76 & 82.93 & 1000 & 967 & 810 \\
\hline 16510 & COSMOS 1727 & 1986-008A & 104.78 & 82.95 & 1013 & 955 & 810 \\
\hline 16727 & cosmos 1745 & 1986-037A & 104.79 & 82.96 & 1008 & 962 & 810 \\
\hline 17066 & COSMOS 1791 & 1986-086A & 104.67 & 82.95 & 1010 & 948 & 810 \\
\hline 17159 & COSMOS 1802 & 1986-093A & 104.9 & 82.93 & 1020 & 960 & 810 \\
\hline 17239 & COSMOS 1808 & $1986-100 \mathrm{~A}$ & 104.96 & 82.93 & 1016 & 969 & 810 \\
\hline 17359 & COSMOS 1816 & 1987-009A & 104.77 & 82.93 & 1008 & 960 & 810 \\
\hline 17525 & COSMOS 1821 & 1987-017A & 104.83 & 82.91 & 1015 & 958 & 810 \\
\hline 18129 & COSMOS 1861 & 1987-054A & 104.88 & 82.92 & 999 & 979 & 810 \\
\hline 18160 & COSMOS 1864 & 1987-057A & 104.69 & 82.93 & 1004 & 956 & 810 \\
\hline 18709 & COSMOS 1904 & 1987-106A & 104.79 & 82.91 & 1005 & 964 & 810 \\
\hline 18985 & cosmos 1934 & 1988-023A & 104.62 & 82.96 & 1007 & 947 & 810 \\
\hline 19324 & cosmos 1959 & $1988-062 A$ & 104.65 & 82.95 & 1002 & 954 & 810 \\
\hline 19826 & COSMOS 2004 & 1989-017A & 104.94 & 82.95 & 1014 & 969 & 810 \\
\hline 19921 & cosmos 2016 & 1989-028A & 104.73 & 82.96 & 1011 & 952 & 810 \\
\hline 20045 & cosmOS 2026 & $1989-042 A$ & 104.65 & 82.94 & 1008 & 948 & 810 \\
\hline 20103 & NADEZHDA 1 & 1989-050A & 104.76 & 82.96 & 1010 & 957 & 825 \\
\hline 20149 & cosmOS 2034 & 1989-059A & 104.85 & 82.94 & 1011 & 964 & 810 \\
\hline 20508 & NADEZHDA 2 & 1990-017A & 104.79 & 82.96 & 1016 & 953 & 825 \\
\hline 20527 & COSMOS 2061 & 1990-023A & 104.95 & 82.94 & 1015 & 969 & 825 \\
\hline 20577 & cosmOS 2074 & $1990-036 \mathrm{~A}$ & 104.76 & 82.94 & 1003 & 963 & 825 \\
\hline 20804 & cosmos 2100 & 1990-083A & 104.78 & 82.94 & 1012 & 956 & 825 \\
\hline 21087 & INFORMATOR 1 & 1991-006A & 104.71 & 82.94 & 1008 & 954 & 825 \\
\hline 21089 & cosmos 2123 & 1991-007A & 104.76 & 82.92 & 1006 & 960 & 825 \\
\hline
\end{tabular}




\begin{tabular}{|c|c|c|c|c|c|c|c|}
\hline 21152 & NADEZHDA 3 & 1991-019A & 104.79 & 82.92 & 1014 & 955 & 825 \\
\hline 21230 & cosmos 2142 & 1991-029A & 104.87 & 82.96 & 1017 & 960 & 825 \\
\hline 21666 & COSMOS 2154 & 1991-059A & 104.86 & 82.9 & 1006 & 970 & 825 \\
\hline 21796 & COSMOS 2173 & 1991-081A & 104.69 & 82.96 & 1017 & 944 & 825 \\
\hline 21875 & cosmos 2180 & 1992-008A & 104.83 & 82.93 & 1015 & 958 & 825 \\
\hline 21902 & COSMOS 2181 & $1992-012 \mathrm{~A}$ & 104.93 & 82.94 & 1012 & 971 & 825 \\
\hline 21937 & COSMOS 2184 & $1992-020 \mathrm{~A}$ & 104.87 & 82.93 & 1012 & 965 & 825 \\
\hline 22006 & cosmos 2195 & 1992-036A & 104.74 & 82.93 & 1010 & 954 & 825 \\
\hline 22207 & COSMOS 2218 & 1992-073A & 104.89 & 82.92 & 1014 & 965 & 825 \\
\hline 22307 & cosmos 2230 & $1993-001 \mathrm{~A}$ & 104.84 & 82.94 & 1004 & 970 & 825 \\
\hline 22487 & cosMOS 2233 & 1993-008A & 104.67 & 82.95 & 1006 & 952 & 825 \\
\hline 22590 & COSMOS 2239 & 1993-020A & 104.7 & 82.93 & 998 & 962 & 825 \\
\hline 22888 & cosmos 2266 & 1993-070A & 104.74 & 82.95 & 1018 & 947 & 825 \\
\hline 23092 & cosmOS 2279 & 1994-024A & 104.63 & 82.95 & 1004 & 950 & 825 \\
\hline 23179 & NADEZHDA 4 & $1994-041 \mathrm{~A}$ & 104.61 & 82.95 & 1001 & 951 & 825 \\
\hline 23189 & COSMOS 2285 & 1994-045A & 104.94 & 74.03 & 1010 & 973 & 825 \\
\hline 23463 & TSIKADA & $1995-002 \mathrm{~A}$ & 104.92 & 82.93 & 1018 & 963 & 825 \\
\hline 23526 & cosmos 2310 & $1995-012 \mathrm{~A}$ & 104.97 & 82.94 & 1010 & 976 & 825 \\
\hline 23603 & cosmos 2315 & 1995-032A & 104.89 & 82.9 & 1012 & 967 & 825 \\
\hline 23773 & cosmos 2327 & 1996-004A & 104.77 & 82.98 & 1020 & 947 & 795 \\
\hline 24304 & cosmos 2334 & $1996-052 A$ & 104.84 & 82.93 & 1010 & 964 & 825 \\
\hline 24677 & cosmos 2336 & 1996-071A & 104.99 & 82.94 & 1012 & 976 & 795 \\
\hline 24772 & cosmos 2341 & 1997-017A & 105 & 82.92 & 1014 & 976 & 795 \\
\hline 24953 & cosmos 2346 & 1997-052A & 104.38 & 82.92 & 994 & 937 & 795 \\
\hline 25567 & NADEZHDA 5 & 1998-072A & 104.97 & 82.95 & 1011 & 975 & 825 \\
\hline 25590 & cosmos 2361 & 1998-076A & 104.89 & 82.94 & 1011 & 967 & 825 \\
\hline 25892 & cosmos 2366 & 1999-045A & 104.77 & 82.93 & 1007 & 960 & 795 \\
\hline 26818 & cosmos 2378 & 2001-023A & 104.81 & 82.93 & 1009 & 962 & 795 \\
\hline 27436 & COSMOS 2389 & $2002-026 \mathrm{~A}$ & 104.73 & 82.95 & 1017 & 947 & 795 \\
\hline 27534 & NADEZHDA 7 & $2002-046 \mathrm{~A}$ & 104.91 & 82.93 & 1018 & 962 & 825 \\
\hline 27818 & cosmOS 2398 & 2003-023A & 104.95 & 82.95 & 1016 & 968 & 795 \\
\hline
\end{tabular}




\begin{tabular}{|c|c|c|c|c|c|c|c|}
\hline 28380 & COSMOS 2407 & $2004-028 \mathrm{~A}$ & 104.65 & 82.96 & 1008 & 948 & 820 \\
\hline 32052 & COSMOS 2429 & $2007-038 \mathrm{~A}$ & 104.74 & 82.98 & 1011 & 953 & 795 \\
\hline \multicolumn{7}{|c|}{ Other Payloads } \\
\hline 12409 & COSMOS 1266 & $1981-037 \mathrm{~A}$ & 103.57 & 64.76 & 963 & 891 & 1295 \\
\hline 12783 & COSMOS 1299 & $1981-081 \mathrm{~A}$ & 103.94 & 65.12 & 968 & 922 & 1295 \\
\hline 13243 & COSMOS 1372 & $1982-052 \mathrm{~A}$ & 103.9 & 64.9 & 973 & 913 & 1237 \\
\hline 10358 & COSMOS 952 & $1977-088 \mathrm{~A}$ & 104.08 & 64.94 & 975 & 928 & 1295 \\
\hline 13600 & COSMOS 1412 & $1982-099 \mathrm{~A}$ & 103.88 & 64.81 & 979 & 905 & 1295 \\
\hline 7005 & COSMOS 626 & $1973-108 \mathrm{~A}$ & 103.95 & 65.44 & 983 & 907 & 1295 \\
\hline 16917 & COSMOS 1771 & $1986-062 \mathrm{~A}$ & 104.16 & 64.98 & 983 & 927 & 1295 \\
\hline 15085 & COSMOS 1579 & $1984-069 \mathrm{~A}$ & 103.89 & 65.05 & 984 & 901 & 1295 \\
\hline 18122 & COSMOS 1860 & $1987-052 \mathrm{~A}$ & 103.99 & 65.01 & 989 & 905 & 1295 \\
\hline 9486 & COSMOS 860 & $1976-103 \mathrm{~A}$ & 104.25 & 64.7 & 989 & 930 & 1295 \\
\hline 15930 & COSMOS 1670 & $1985-064 \mathrm{~A}$ & 104.07 & 64.94 & 991 & 910 & 1295 \\
\hline 18957 & COSMOS 1932 & $1988-019 \mathrm{~A}$ & 104.37 & 65.04 & 994 & 937 & 1295 \\
\hline 6206 & COSMOS 521 & $1972-074 \mathrm{~A}$ & 104.92 & 65.83 & 995 & 987 & 750 \\
\hline 5050 & COSMOS 400 & $1971-020 \mathrm{~A}$ & 104.95 & 65.83 & 997 & 987 & 750 \\
\hline 12149 & COSMOS 1241 & $1981-006 \mathrm{~A}$ & 104.94 & 65.82 & 997 & 986 & 750 \\
\hline
\end{tabular}


$11869,12442,12791,13027,13148,13241,13648,13991,14240, \ldots$ $14401,14759,15031,15482,16011,16681,16860,16952,17303, \ldots$ $18095,18585,19038,19256,19769,21014,22675,23431]$;

cluster_alt $=7146 ; \% \mathrm{~km}$

cluster_inc $=74 ; \%$ deg

elseif cluster $==2$

\% SL-16 Cluster with Plds and Miscellaneous Debris (850 km) (75 objects) $\mathrm{SSC}=[16182,16181,22285,22284,23405,23404,22220,22219, \ldots$ $31793,31792,28353,28352,23088,23087,19650,19649,22803, \ldots$ $22802,23705,23704,26070,26069,20625,20624,22566,22565, \ldots$ $17974,17973,17590,17589,25407,25406,24298,24297,19120, \ldots$ $19119,08519,07574,04419,11288,05731,10514,07209,08026, \ldots$ 09481, 07274, 11605, 06659, 08799, 06392, 13718, 07490, 08845, . . 06256, 11962, 05917, 07714, 09661, 06079, 09903, 12456, 11165, . . $15334,15772,32958,20322,37214,22823,37849,15427,19531, \ldots$ 28654, 11166, 13719, 11289] ;

cluster_a7t $=7210 ; \% \mathrm{~km}$

cluster_inc $=71 ; \%$ deg

elseif cluster $==3$

\% SL-8 Cluster ( $975 \mathrm{~km}$ Cluster) (304 objects)

$\mathrm{SSC}=[04784,04800,05239,05685,05847,06020,06149,06207, \ldots$ 06708, 06829, 07009, 07095, 07350, 07477, 07594, 07737, 07769, . . 08073, 08421, 08597, 08646, 08874, 09044, 09062, 09510, 09613, .. $09638,09738,09848,10020,10138,10142,10355,10461,10513, \ldots$ $10537,10600,10693,10732,10745,10777,10918,10992,11239, \ldots$ $11309,11321,11327,11379,11586,11668,11681,11736,11751, \ldots$ $11804,12088,12092,12298,12508,12682,12836,13034,13066, \ldots$ $13111,13128,13260,13618,13758,13917,13950,14059,14085, \ldots$ $14451,14547,14625,14680,14966,14974,15056,15078,15293, \ldots$ $15360,15399,15506,15598,15752,16292,16369,16494,16511, \ldots$ $16728,17067,17160,17240,17360,17526,18130,18161,18710, \ldots$ $18986,19325,19827,19922,20046,20104,20150,20509,20528, \ldots$ $20578,20805,21088,21090,21153,21231,21667,21797,21876, \ldots$ $21903,21938,22007,22208,22308,22488,22591,22889,23093, \ldots$ $23180,23190,23466,23527,23604,23774,24306,24678,24773, \ldots$ $24955,25569,25592,25893,26819,27437,27535,27819,28381, \ldots$ 32053, 04783, 04799, 05238, 05683, 05846, 06019, 06148, 06206, . . 06707, 06828, 07008, 07094, 07349, 07476, 07593, 07736, 07768, . . 08072, 08419, 08591, 08645, 08873, 09025, 09061, 09509, 09610, . . 09637, 09737, 09846, 10019, 10137, 10141, 10352, 10459, 10512, . . $10536,10599,10692,10731,10744,10776,10917,10991,11238, \ldots$ $11308,11320,11326,11378,11667,11680,11735,11750,11803, \ldots$ $12087,12091,12297,12504,12681,12835,13033,13065,13110, \ldots$ $13127,13259,13757,13916,13949,14057,14084,14450,14546, \ldots$ $14624,14679,14965,14973,15055,15077,15292,15359,15398, \ldots$ $15505,15597,15751,16291,16368,16493,16510,16727,17066, \ldots$ $17159,17239,17359,17525,18129,18160,18709,18985,19324, \ldots$ $19826,19921,20045,20103,20149,20508,20527,20577,20804, \ldots$ $21087,21089,21152,21230,21666,21796,21875,21902,21937, \ldots$ $22006,22207,22307,22487,22590,22888,23092,23179,23189, \ldots$ 
$23463,23526,23603,23773,24304,24677,24772,24953,25567, \ldots$

$25590,25892,26818,27436,27534,27818,28380,32052,12409, \ldots$

$12783,13243,10358,13600,07005,16917,15085,18122,09486, \ldots$

$15930,18957,05050,12149]$;

cluster_a7t $=7346 ; \% \mathrm{~km}$

cluster_inc $=83 ; \%$ deg

end

\section{Open STK and connect}

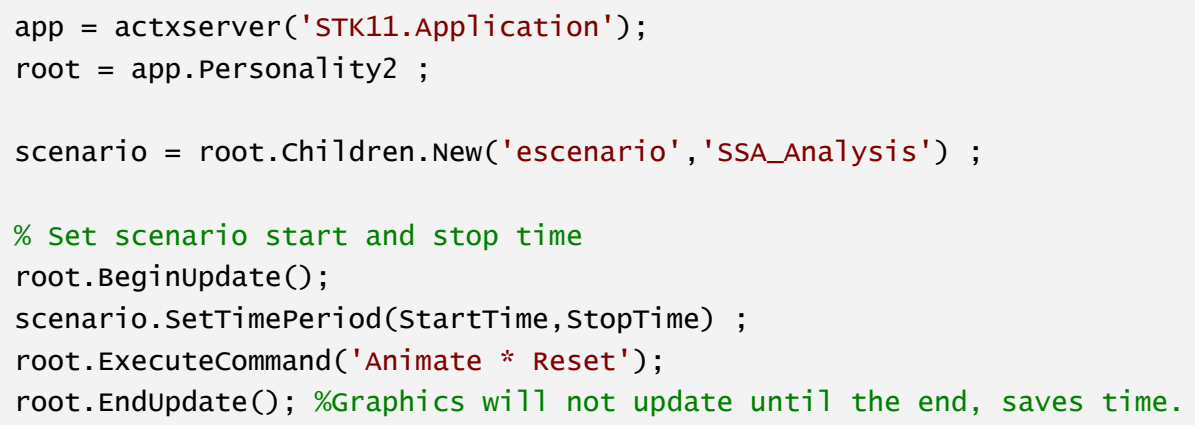

\section{Define data storage structure:}

conjunctiondata = struct ('Primarysate11ite', \{\} , 'ConjunctionInfo', \{\} );

conjInfostruct $=$ struct ('Conjunctingsatel1ite', \{\}, 'TCA' $^{\prime}\{\}$, 'RangeMag', \{\}$, \ldots$

'Radial', \{\}, 'InPlane', \{\}, 'Norma1', \{\}, 'VelocityMag', \{\}, 'V_Radial', \{\}$, \ldots$

'V_InPlane', \{\}, 'V_Normal', \{\}, 'Longitude', \{\}, 'Latitude', \{\}, 'Altitude', \{\}$, \ldots$

'Accessstart', \{\} , 'Accessstop', \{\} , 'Duration', \{\} );

subptElems = \{'Lat'; 'Lon'; 'ATt' $\}$;

\section{RSO Initialization}

Load the satellites into STK.

$\mathrm{h}=$ waitbar(0, 'RSO Initialization') ;

\% Change time to Epoch Seconds

root.UnitPreferences. Item('DateFormat') . SetCurrentunit( 'EPSEC');

root. Beginupdate();

for $i=1$ : 1ength(SSC)

\% Grab the satellite ID

sscinc $=\operatorname{ssc}(i)$;

\% Load satellite in STK

sate11ite= scenario.Chi1dren. New('esate11ite', num2str(SSCinc, '\%05d')) ;

cmd = ['SetState */Sate11ite/' num2str(SSCinc, '\%05d') ' SGP4 "', startTime,...

'" "', StopTime,'" 60 ' num2str(SSCinc, \%05d') ' TLESource Automatic'] ;

root. ExecuteCommand (cmd); 


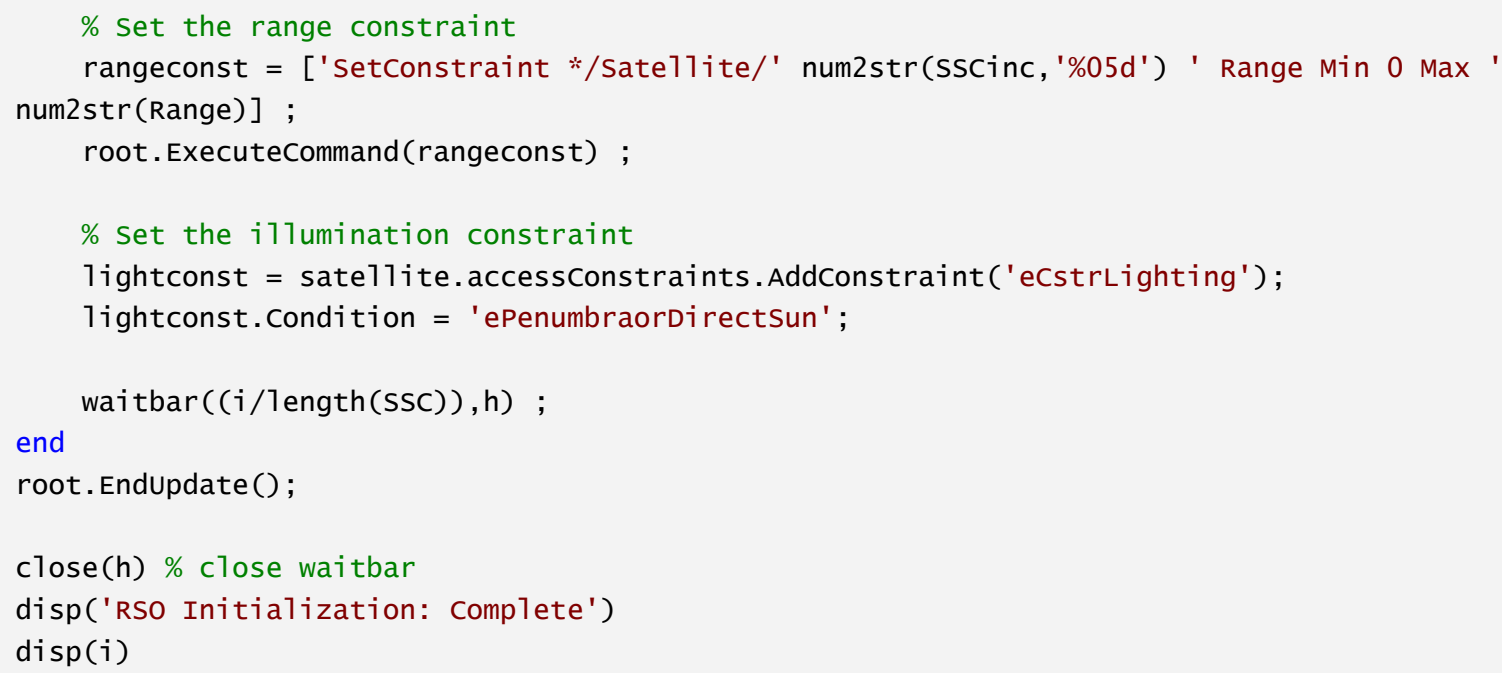

OBS Initialization

Load the constellation into STK.

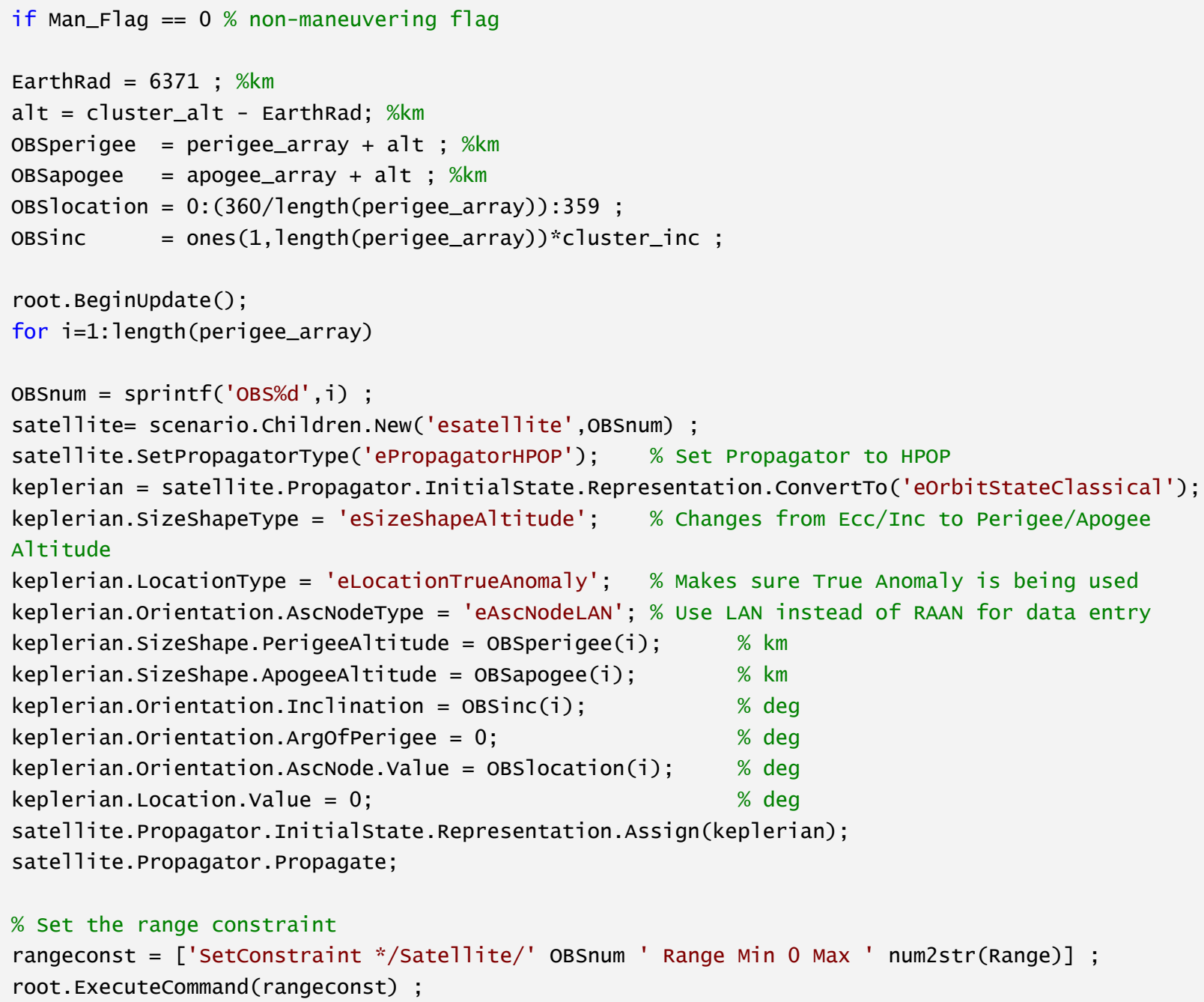


end

root. Endupdate();

disp('OBS Initialization: Complete')

$\operatorname{disp}(i)$

end

\section{Maneuver Manager 1}

\% Phasing Maneuver

if Man_Flag == $1 \%$ phasing maneuver flag

alt = cluster_alt; \%km

OBSperigee = perigee_array + alt ; \%km

OBSapogee = apogee_array + a1t ; \%km

OBS1ocation $=0:(360 /$ length (perigee_array $)): 359 ;$

OBSinc = ones ( 1 , length(perigee_array))*cluster_inc ;

circular_con $=$ (OBSperigee $(1)==$ OBSapogee $(1))$;

for $i=1$ : length(OBS1ocation)

$\%$ start satellite

OBSnum = sprintf('OBS\%d',i);

sate11ite= scenario.Chi1dren. New('esate11ite', obsnum);

\% start waitbar

OBSstring = strcat (OBSnum,' Loading Maneuvers');

h2 = waitbar(0, OBSstring);

$\%$ set propagator type to Astrogator

sate11ite.SetPropagatorType('ePropagatorAstrogator');

ASTG = satel1ite. Propagator $;$

ASTG.Mainsequence.RemoveA11(); \%Clear a11 segments from the MCS

\% Insert Initial State segment into MCS and configure

initState = ASTG.Mainsequence.Insert('eVASegmentTypeInitialstate', 'Initial State', '-');

initstate. Initialstate. . ppoch = scenario. StartTime;

initstate. SetElementType ('eVAElementTypeKeplerian');

kep = initstate. Element;

kep.PeriapsisRadiussize $=$ oBSperigee $(i)$;

kep. Eccentricity $=1-2 /($ OBSapogee $(i) /$ oBSperigee $(i))+1)$;

kep. ArgofPeriapsis $=0$;

kep. RAAN $=$ oBSlocation $(i)$;

kep. Inclination $=\operatorname{OBSinc}(i)$;

kep. TrueAnomaly $=0$;

\% Propagate to start

propagate1 = ASTG.Mainsequence. Insert('eVASegmentTypePropagate', 'Propagate 1', '-');

propagate1. PropagatorName = 'Earth Point Mass';

propagate1. Properties.Color $=255 ; \%$ Red 
propagate1.StoppingConditions.Item('Duration'). Properties.Trip $=3600 * 24 * 10$;

if circular_con

\% Insert a Propagate state segment into the MCS and Add/Remove stopping Conditions

propagate2 = ASTG.Mainsequence. Insert('eVASegmentTypePropagate', 'Propagate 2', '-');

propagate2. PropagatorName = 'Earth Point Mass';

propagate2. Properties.Color $=255$; \% Red

propagate2.StoppingConditions.Add ('AscendingNode');

propagate2.StoppingConditions.Remove('Duration');

else

\% Insert a Propagate state segment into the MCS and Add/Remove Stopping Conditions

propagate2 = ASTG.Mainsequence. Insert('eVASegmentTypePropagate', 'Propagate 2', '-');

propagate2. PropagatorName = 'Earth Point Mass';

propagate2. Properties.Color $=255$; \% Red

propagate2.StoppingConditions.Add ('Apoapsis ') ;

propagate2.StoppingConditions.Remove ('Duration');

end

Propcount $=2$;

$\%$ Run the MCS

ASTG. RunMCS;

$\%$ set the range constraint

rangeconst $=[$ 'SetConstraint */Sate11ite/' OBSnum ' Range Min 0 Max ' num2str(Range)]; root. Executecommand (rangeconst) ;

$\%$ Get time of apoapsis

EndTime = satel1ite.Propagator. Mainsequence.Item(2).Finalstate. Epoch ;

EndTime_old = scenario.StartTime ;

1oop_count $=1 ; \%$ Initialize 1oop counter

while(1)

$\%$ check access to see if burn is needed

Conjcount $=0$;

for $i d x=1:$ length (SSC)

sat1 = scenario. Chi1dren. Item (num2str $\left.\left(\operatorname{Ssc}(i d x),{ }^{\prime} \% 05 d^{\prime}\right)\right)$;

acc=sate11ite. GetAccessToobject (sat1);

acc. AccessTimePeriod = 'eUserSpecAccessTime';

acc. AccessTimePeriodData. AccessInterval. State = 'ecrdnSmartIntervalstatestartstop';

accStartEpoch = acc.AccessTimePeriodData.AccessInterva1.GetStartEpoch();

accStartEpoch.SetExplicitTime(EndTime_old) ;

acc. AccessTimePeriodData.AccessInterva1. SetStartEpoch (accstartEpoch);

accStopEpoch = acc.AccessTimePeriodData.AccessInterva1.GetStopEpoch();

accStopEpoch.SetExplicitTime(EndTime) ;

acc. AccessTimePeriodData.AccessInterva1. SetStopEpoch (accStopEpoch);

acc. ComputeAccess;

accessDP $=$ acc.DataProviders.Item ('Access Data').Exec(EndTime_old, EndTime) ;

if acc. ComputedAccessintervalTimes. Count $\sim=0$

Conjcount $=$ ConjCount + acc.ComputedAccessIntervalTimes. Count ;

$\operatorname{Duration}(\mathrm{idx})=$

sum (ce112mat (accessDP.DataSets.GetDataSetByName ('Duration') .Getvalues)) ;

end 


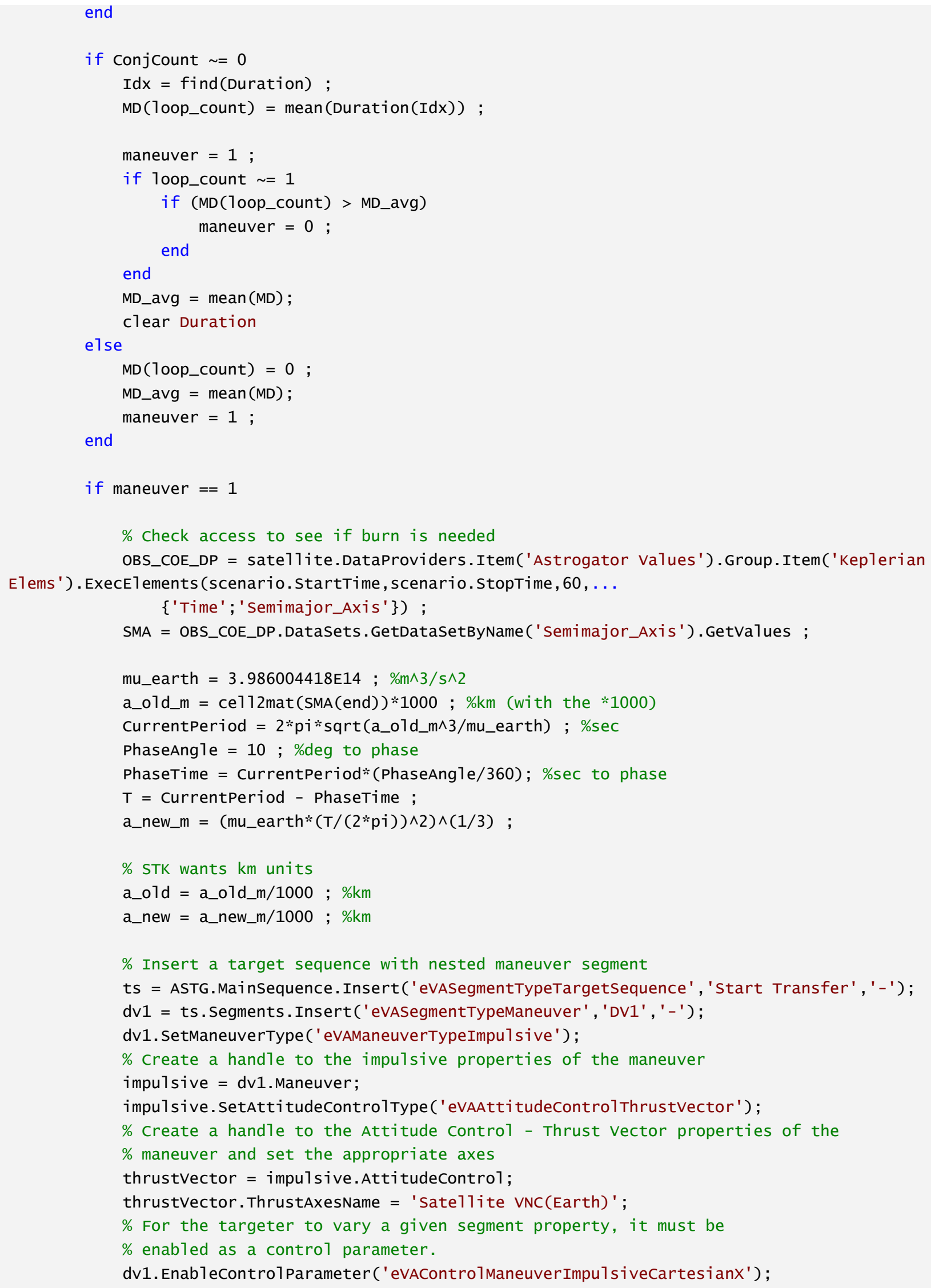


\% Segment Results, which can be used as targeter goals, are also stored in a collection

dv1.Results.Add('Keplerian Elems/Semimajor Axis');

\% Targter Profiles are also stored as a collection

dc = ts.Profiles.Item('Differential Corrector');

\% create a handle to the targeter control and set its properties

xControlParam = dc. ControlParameters.GetControlByPaths('DV1',

'Impulsivemnvr. Cartesian. $X$ ');

xcontrolParam. Enable = true;

xControlparam. Maxstep $=0.3$;

\% create a handle to the targeter results and set its properties

Result = dc.Resu1ts.GetResultByPaths('DV1', 'Semimajor Axis');

Result. Enable = true;

Resu1t.Desiredvalue = a_new ;

Result. Tolerance $=0.1$;

$\%$ Set final DC and targeter properties and run modes

dc. Maxiterations $=50$;

dc. Enabledisplaystatus = true;

dc. Mode = 'evAProfilemodeIterate' ;

ts. Action = 'evATargetSeqActionRunActiveProfiles';

\% Propagate the Transfer orbit to Apogee

transferE11ipse = ASTG. MainSequence. Insert('eVASegmentTypePropagate' , 'Transfer

E11ipse', '- ');

transferE17ipse. PropagatorName = 'Earth Point Mass';

propagate2. Properties. Color $=65280$;

\% Add an Apoapsis Stopping Condition and remove the Duration Stopping Condition

transferE11ipse.StoppingConditions.Add ('Apoapsis');

transferE17ipse.StoppingConditions.Remove('Duration');

if circular_con

\% Propagate the Transfer orbit to Apogee

transferEl1ipse = ASTG. Mainsequence. Insert('eVASegmentTypePropagate', 'Transfer

E11ipse', '- ');

transferE11ipse. PropagatorName = 'Earth Point Mass';

propagate2. Properties. Color $=65280$;

\%Add an Apoapsis stopping Condition and remove the Duration Stopping Condition transferE11ipse.StoppingConditions.Add ('AscendingNode');

transferE11ipse.StoppingConditions.Remove('Duration');

else

\% Propagate the Transfer orbit to Apogee

transferE11ipse = ASTG. MainSequence. Insert ('eVASegmentTypePropagate' , 'Transfer

E11ipse', '- ');

transferE11ipse.PropagatorName = 'Earth Point Mass';

propagate2. Properties. Color $=65280$;

\%Add an Apoapsis Stopping Condition and remove the Duration Stopping Condition

transferEl1ipse.StoppingConditions.Add('Apoapsis');

transferE11ipse.StoppingConditions.Remove('Duration');

end

\% Maneuver into the Outer orbit 


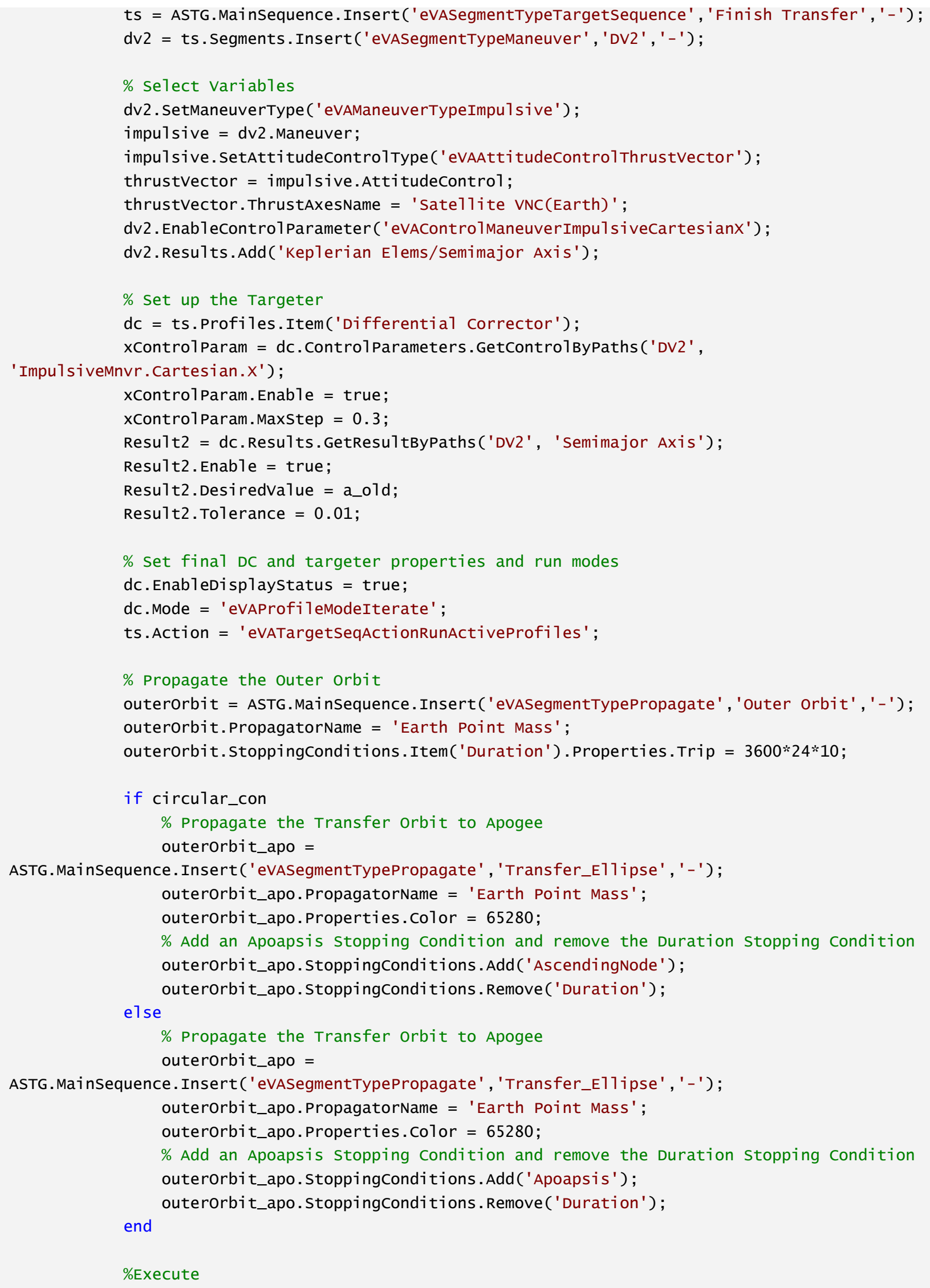


ASTG. RunMCS;

else

\% Propagate

Propcount $=$ Propcount +1 ;

prop_string $=[$ 'Propagate' num2str(PropCount) $]$;

propagate = ASTG.MainSequence.Insert('eVASegmentTypePropagate', prop_string, '-');

propagate. PropagatorName = 'Earth Point Mass';

propagate.StoppingConditions. Item('Duration'). Properties.Trip $=3600 * 24 * 10$;

if circular_con

\% Propagate the Transfer orbit to Apogee

outerorbit_apo $=$

ASTG. Mainsequence. Insert ('eVASegmentTypePropagate ' , 'Transfer_E11 ipse' , ' - ') ; outerorbit_apo.PropagatorName = 'Earth Point Mass'; outerorbit_apo.Properties.Color $=65280$;

\% Add an Apoapsis Stopping Condition and remove the Duration stopping Condition outerorbit_apo.StoppingConditions.Add ('AscendingNode') ;

outerorbit_apo.StoppingConditions.Remove('Duration');

e1se

\% Propagate the Transfer orbit to Apogee

outerorbit_apo =

ASTG.Mainsequence. Insert (' eVASegmentTypePropagate ' , 'Transfer_E11 ipse' , ' - ') ;

outerorbit_apo.PropagatorName = 'Earth Point Mass';

outerorbit_apo.Properties.Color $=65280$;

\% Add an Apoapsis Stopping Condition and remove the Duration stopping Condition outerorbit_apo.StoppingConditions.Add('Apoapsis');

outerorbit_apo.StoppingConditions.Remove('Duration');

end

\% Execute

ASTG. RunMCS;

end

$\%$ Get end time

EndTime_old = EndTime ;

count = sate11ite. Propagator. Mainsequence. count;

EndTime = sate11ite. Propagator. Mainsequence. Item(count-1). Fina1State. Epoch ;

waitbar ((EndTime/ScenarioLength), h2, oBSstring) ;

if EndTime > (ScenarioLength- $(3600 * 24))$

break

end

1oop_count = 1oop_count+1;

end

propagate_end = ASTG.MainSequence.Insert('eVASegmentTypePropagate', 'Propagate End', '-'); propagate_end.PropagatorName = 'Earth Point Mass';

propagate_end.StoppingConditions.Add ('Epoch');

propagate_end.StoppingConditions.Remove('Duration'); 
propagate_end.StoppingConditions.Item('Epoch').Properties.Trip = ScenarioLength;

ASTG . RunMCS;

\% close waitbar

close (h2)

end

end

\section{Maneuver Manager 2}

\% RAAN Maneuver

if Man_Flag $==2 \%$ maneuver flag

alt = cluster_alt; \%km

OBSperigee = perigee_array + alt ; \%km

OBSapogee = apogee_array + a7t ; \% km

OBS1ocation $=0:(360 /$ length (perigee_array $)): 359 ;$

OBSinc = ones(1, length(perigee_array))*cluster_inc ;

for $i=1$ : length(OBSlocation)

\% start satellite

OBSnum = sprintf('OBS\%d',i) ;

sate11ite= scenario. Children. New('esate11ite', obsnum);

\% start waitbar

OBSstring = strcat (OBSnum,' Loading Maneuvers');

h2 = waitbar(0, OBSstring);

\% set propagator type to astrogator

sate11ite.SetPropagatorType('ePropagatorAstrogator');

ASTG = satel1ite. Propagator $;$

ASTG.Mainsequence.RemoveA11(); \%Clear a11 segments from the MCS

\% Insert Initial State segment into MCS and configure

initState = ASTG.Mainsequence.Insert('eVASegmentTypeInitialstate', 'Initial State', '-');

initstate. Initialstate. Epoch = scenario.StartTime;

initstate. SetElementType ('eVAElementTypeKeplerian');

kep = initstate. Element;

kep.PeriapsisRadiussize $=$ oBsperigee $(i)$;

kep. Eccentricity $=1-2 /($ OBSapogee $(i) /$ oBSperigee $(i))+1)$;

kep. Argofperiapsis $=0$;

kep. RAAN $=$ oBSlocation $(i)$;

kep. Inclination $=\operatorname{OBSinc}(i)$;

kep. TrueAnomaly $=0$;

\% Propagate for a ful1 day

propagate1 = ASTG.Mainsequence. Insert('eVASegmentTypePropagate', 'Propagate 1', '-');

propagate1. PropagatorName = 'Earth Point Mass';

propagate1.Properties.Color $=255 ; \%$ Red 
propagate1.StoppingConditions.Item('Duration'). Properties.Trip $=3600 * 24 * 5$;

dRAAN $=5 ;$

$v u=\operatorname{acosd}(\operatorname{cosd}($ OBSinc $(i)) \wedge 2+\operatorname{sind}($ OBSinc $(i)) \wedge 2 * \cos d($ dRAAN $)) ;$

ArgLat_i $=\operatorname{acosd}(\operatorname{tand}(\operatorname{OBSin} c(i)) *((\operatorname{cosd}(\operatorname{drAAN})-\operatorname{cosd}(v u)) /(\operatorname{sind}(v u))))$;

ArgLat_f $=\operatorname{acosd}(\operatorname{cosd}($ OBSinc $(i)) * \operatorname{sind}(\operatorname{OBSinc}(i)) *((1-\operatorname{cosd}(\operatorname{drAAN})) /(\operatorname{sind}(\operatorname{vu}))))$;

\% Insert a Propagate State segment into the MCS and Add/Remove Stopping Conditions

propagate2 = ASTG.Mainsequence.Insert('eVASegmentTypePropagate', 'Propagate 2', '-');

propagate2. PropagatorName = 'Earth Point Mass';

propagate2. Properties.Color $=255$; \% Red

propagate2.StoppingConditions.Add('Argument of Latitude');

propagate2.StoppingConditions.Remove('Duration');

propagate2.StoppingConditions.Item('Argument_of_Latitude').Properties.Trip = ArgLat_i ;

Propcount $=2$;

\% Run the MCS

ASTG . RunMCS;

$\%$ set the range constraint

rangeconst $=[$ 'SetConstraint */Sate11ite/' OBSnum ' Range Min 0 Max ' num2str(Range)]; root. Executecommand (rangeconst) ;

$\%$ Get time of apoapsis

EndTime = satel1ite.Propagator. MainSequence.Item(2).Finalstate. Epoch ;

1oop_count = 1 ;

maneuver=1;

while(1)

if maneuver $==1$

\% Insert a target sequence with nested maneuver segment

ts = ASTG.Mainsequence. Insert ('eVASegmentTypeTargetSequence', 'Start Transfer' , '- ');

dv1 = ts. Segments. Insert ('eVASegmentTypeManeuver' , 'DV1' , ' - ');

dv1. SetManeuverType ('evaManeuverTypeImpulsive');

$\%$ create a handle to the impulsive properties of the maneuver

impulsive = dv1. Maneuver;

impulsive.SetAttitudeControlType ('evaAttitudecontrolThrustVector') ;

$\%$ create a handle to the Attitude control - Thrust vector properties of the

$\%$ maneuver and set the appropriate axes

thrustvector = impulsive. Attitudecontrol;

thrustvector. ThrustAxesName = 'Sate11ite VNC(Earth)';

$\%$ For the targeter to vary a given segment property, it must be

$\%$ enabled as a control parameter.

dv1. Enablecontrol Parameter ('evAControlManeuverImpulsivecartesianX') ;

dv1. Enablecontrol Parameter ('evAControlManeuver Impulsivecartesiany') ;

$\%$ segment Results, which can be used as targeter goals, are also stored in a

collection

dv1.Resu1ts.Add ('Keplerian Elems/Argument of Latitude') ;

dv1.Resu1ts.Add('Keplerian Elems/Radius of Apoapsis');

$\%$ Targter Profiles are also stored as a collection

dc = ts.Profiles.Item('Differential Corrector'); 


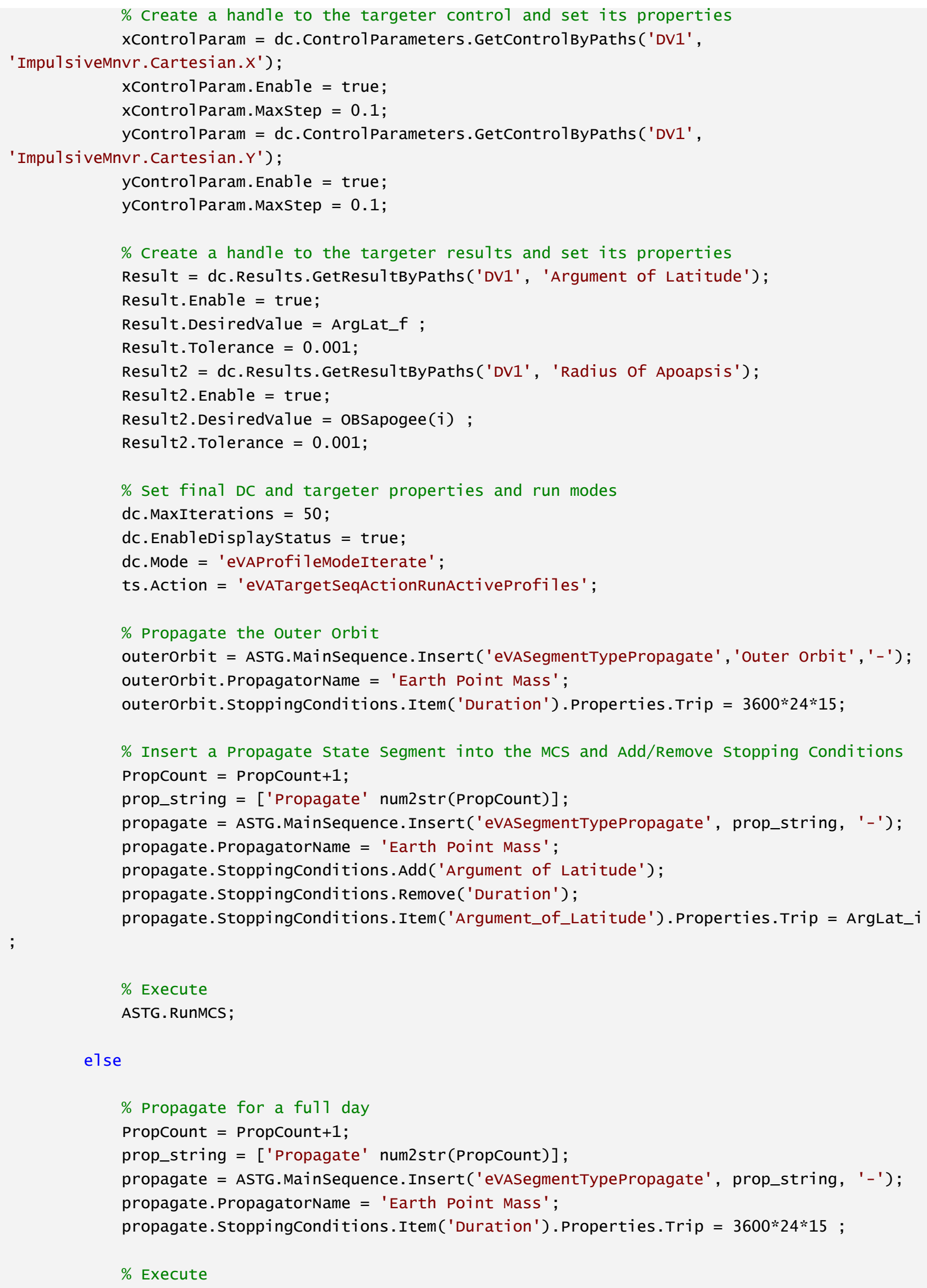




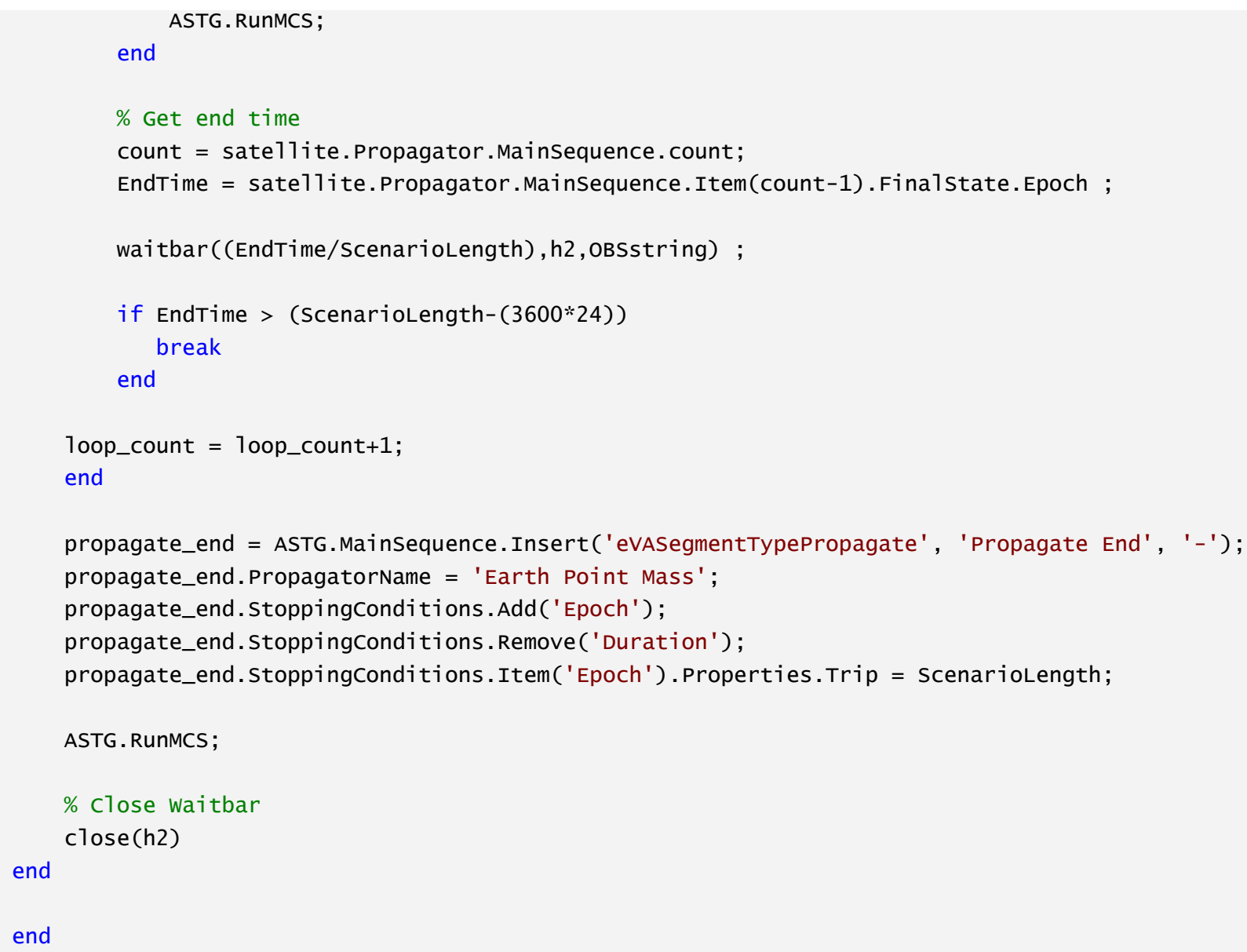

\section{Conjunction Data Generation}

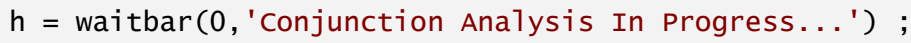

for $i=1$ : length (SSC)

Sscinc $=\operatorname{Ssc}(i)$

sat1 = scenario.Chi1dren.Item (num2str(SSCinc, '\%05d')) ;

\%set up data storage, in structure, for current satel1ite

conjunctionData(i). Primarysatellite = sscinc;

conjunctionData (i). ConjunctionInfo = conjinfostruct;

\% Compare to each other satellite in SSC vector

numconjunctions $=0$;

for $j=1: 1$ ength (apogee_array) \%1ength(OBS)

OBSinc $=$ sprintf('OBS\%d',j);

sat2 = scenario. Children. Item(oBsinc);

acc=sat1. GetAccessToobject (sat2);

acc. ComputeAccess ;

\% create displacement vector between the two satellites and a

\% corresponding vector Magnitude calculation scalar to query the 
$\%$ time of min and min range values:

if (sat1.vgt.Vectors.Contains ('ToSat2') ==0)

relativePositionvector $=$ sat1.vgt.Vectors.Factory.Create ('ToSat2' , 'Displacement

vector', 'ecrdnvectorTypeDisplacement');

else

relativePositionVector $=$ sat1.Vgt.Vectors.Item('ToSat2');

end

relativePositionvector.origin.SetPoint(sat1.Vgt.Points.Item('Center'));

\% create vector parameter set in the RIC frame to get access to the

$\%$ vector components:

if (sat1.vgt.Parametersets.Contains ('ToSat2_RIC') $==0$ ) positionvectorsetRIC =

sat1.vgt.ParameterSets. Factory.CreateParametersetVector('ToSat2_RIC', 'Vector parameter set expressing relative position in the RIC frame');

else

positionvectorSetRIC = sat1.Vgt.ParameterSets.Item('ToSat2_RIC');

end

positionvectorSetRIC.Vector = relativePositionvector;

positionvectorSetRIC. ReferenceAxes = sat1.Vgt.Axes.Item('RIC');

relativePositionMagnitude $=$

positionvectorSetRIC.EmbeddedComponents.Item('ToSat2_RIC.Cartesian.Magnitude'); relativeposition $\mathrm{X}=$

positionvectorSetRIC.EmbeddedComponents.Item('ToSat2_RIC.Cartesian.X'); relativePositionY $=$

positionvectorSetRIC.EmbeddedComponents.Item('ToSat2_RIC.Cartesian.Y') ; relativepositionz $=$

positionVectorSetRIC.EmbeddedComponents.Item('ToSat2_RIC.Cartesian.Z');

$\%$ create a velocity vector by taking the derivative of the position

$\%$ vector in ECEF:

if (sat1.vgt.Vectors.Contains('dxToSat2') ==0)

relativevelocityvector = sat1.vgt.vectors.Factory.Create('dxToSat2', 'Derivative of

relative position vector', 'ecrdnvectorTypeDerivative');

relativeVelocityvector.vector.SetVector(relativePositionvector);

relativeVelocityvector. ReferenceAxes.SetAxes (root.Vgt. We11knownAxes. Earth. Fixed); relativeVelocityvector. DifferencingTimestep $=0.1$;

else

relativevelocityvector $=$ sat1.Vgt.Vectors.Item $\left({ }^{\prime d x T o S a t 2}{ }^{\prime}\right)$;

end

\% create vector parameter set in the RIC frame to get access to the

$\%$ vector components:

if (sat1.vgt.Parametersets.Contains ('dxToSat2_RIC') ==0)

velocityvectorsetRIC $=$

sat1.vgt.Parametersets.Factory.CreateParameterSetVector('dxToSat2_RIC', 'Vector parameter set expressing relative position in the RIC frame');

e1se

end

velocityvectorSetRIC $=$ sat1.Vgt.ParameterSets.Item('dxToSat2_RIC');

velocityvectorSetRIC. Vector = relativeVelocityvector;

velocityvectorSetRIC. ReferenceAxes = sat1.vgt.Axes.Item('RIC'); 
relativevelocityMagnitude $=$

velocityvectorSetRIC. EmbeddedComponents. Item('dxToSat2_RIC.Cartesian.Magnitude') ; relativevelocityx $=$

velocityvectorSetRIC.EmbeddedComponents.Item('dxToSat2_RIC.Cartesian.X'); relativeVelocityY $=$

velocityvectorSetRIC.EmbeddedComponents.Item('dxToSat2_RIC.Cartesian.Y'); relativevelocityz $=$

velocityvectorSetRIC.EmbeddedComponents.Item('dxToSat2_RIC.Cartesian.Z');

\% create an event array to find the time of minimum range per access interval:

if (sat1.vgt. EventArrays. Contains ('TimesofMinRange') $==0$ ) extremaArray $=$

sat1.vgt.EventArrays. Factory.CreateEventArrayExtrema('TimesofMinRange','Times for min range over access intervals');

extremaArray. ExtremumType = 'ecrdnExtremumminimum';

extremaArray.Calculation = relativePositionMagnitude;

extremaArray. IsGlobal = true;

e1se

extremaArray = sat1.vgt.EventArrays.Item('TimesofMinRange');

end

subptDP = sat1. DataProviders.GetDataPrVTimeVarFromPath('LLA State/Fixed');

if acc. ComputedAccessintervalTimes. Count $\sim=0$

$\%$ update the end point of the position vector to the current

$\%$ satellite and pass the access intervals to the event array:

relativePositionVector.Destination.SetPoint(sat2.vgt.Points.Item('Center'));

interva1cmd $=$ ['TimeTool * Sate11ite/', sat1. InstanceName, ' Modify "Time Array"

TimesofMinRange "Times of Extrema" "TimeIntervalList" "Access/Sate11ite-', sat1. InstanceName, '-To-

Sate11ite-', sat2. InstanceName, ' Accessinterva1s"'];

root. Executecommand (intervalcmd);

1oca1MinTimes = extremaArray.FindTimes().Times;

\% Compute Duration of Access Intervals

accDP $=$ acc. . DataProviders. Item ('Access

Data').Exec(scenario.StartTime, scenario.stopTime) ;

DurationstartTimes = accDP.Datasets.GetDataSetByName('start Time').GetValues ;

DurationstopTimes = accDP.Datasets.GetDatasetByName('Stop Time').GetValues ;

DurationTimes = accDP.DataSets.GetDatasetByName('Duration').Getvalues ;

for nRows $=1$ : 1ength (1ocalMinTimes)

numconjunctions $=$ numconjunctions +1 ;

minTime = localMinTimes $\{$ nRows $\}$;

\% Evaluate the relative range and velocity at the

$\%$ minimum range time:

rangeAtMinTime = relativePositionMagnitude. Evaluate (minTime). value;

rangeXAtMinTime = relativePositionX.Evaluate(minTime).Value;

rangeYAtMinTime = relativePositionY.Evaluate(minTime). value;

rangeZAtMinTime $=$ relativePositionz. Evaluate $($ minTime) $)$ Value;

velocityAtMinTime = relativeVelocityMagnitude. Evaluate (minTime). value;

velocityXAtMinTime = relativeVelocityx. Evaluate (minTime). Value;

velocityYAtMinTime $=$ relativeVelocityY. Evaluate (minTime). value; 
velocityzAtMinTime $=$ relativeVelocityz. Evaluate (minTime) . value;

subptRes = subptDP. ExecSingleElements (minTime, subptElems);

deticLatitude $=\operatorname{ce112mat}$ (subptRes. Datasets.GetDataSetByName ('Lat'). GetValues);

deticLongitude = ce112mat (subptRes.DataSets.GetDataSetByName ('Lon'). GetVa1ues);

wgs84A7t = ce112mat (subptRes.DataSets.GetDataSetByName('A1t').GetValues);

\% Populate conjunction data structure:

conjunctionData $(i)$. ConjunctionInfo(numConjunctions). Conjunctingsate11ite $=$

num2str(OBSinc);

conjunctionData(i). ConjunctionInfo(numConjunctions). TCA = minTime;

conjunctionData(i). ConjunctionInfo(numConjunctions). RangeMag = rangeAtMinTime;

conjunctionData(i). ConjunctionInfo(numConjunctions). Radial = rangeXAtMinTime;

conjunctionData(i). ConjunctionInfo(numConjunctions).InPlane = rangeYAtMinTime;

conjunctionData(i). ConjunctionInfo(numConjunctions). Normal = rangeZAtMinTime;

velocityAtMinTime;

conjunctionData(i). ConjunctionInfo(numConjunctions). velocityMag =

velocityXAtMinTime;

conjunctionData(i). ConjunctionInfo(numConjunctions). v_Radial =

velocityYATMinTime;

conjunctionData(i). ConjunctionInfo(numConjunctions). __InPlane =

velocityzatMinTime;

conjunctionData(i). ConjunctionInfo(numConjunctions). v_Normal =

conjunctionData(i). ConjunctionInfo(numConjunctions). Latitude = deticLatitude; conjunctiondata( $i$ ). ConjunctionInfo(numConjunctions). Longitude = deticLongitude; conjunctiondata(i).ConjunctionInfo(numConjunctions).A1titude $=$ wgs $84 \mathrm{~A} 7 \mathrm{t}$;

conjunctionData(i). ConjunctionInfo(numConjunctions). Accessstart =

ce112mat (DurationstartTimes (nRows)) ;

conjunctionData(i). ConjunctionInfo(numConjunctions). Accessstop =

ce112mat (DurationstopTimes (nRows));

conjunctionData(i). ConjunctionInfo(numConjunctions). Duration=

ce112mat (DurationTimes(nRows));

end

end

end

\% Remove computed access to free up memory

acc. RemoveAccess;

\% Remove created components before moving on to next object:

sat1.vgt.Calcscalars. Remove (positionvectorsetRIC. Name);

sat1.vgt. CalcScalars. Remove (veloci tyvectorSetRIC. Name);

sat1.vgt. EventArrays. Remove (extremaArray. Name);

sat1.vgt.vectors. Remove (relativePositionvector. Name);

sat1.Vgt. Vectors. Remove (relativeVelocityvector. Name);

clear acc;

waitbar (i/length (SSC), h) ;

fprintf('sat \%d Complete $\backslash n$ ',i) 
end

close (h)

disp('Data Generation: Complete')

\section{Post Process of Results}

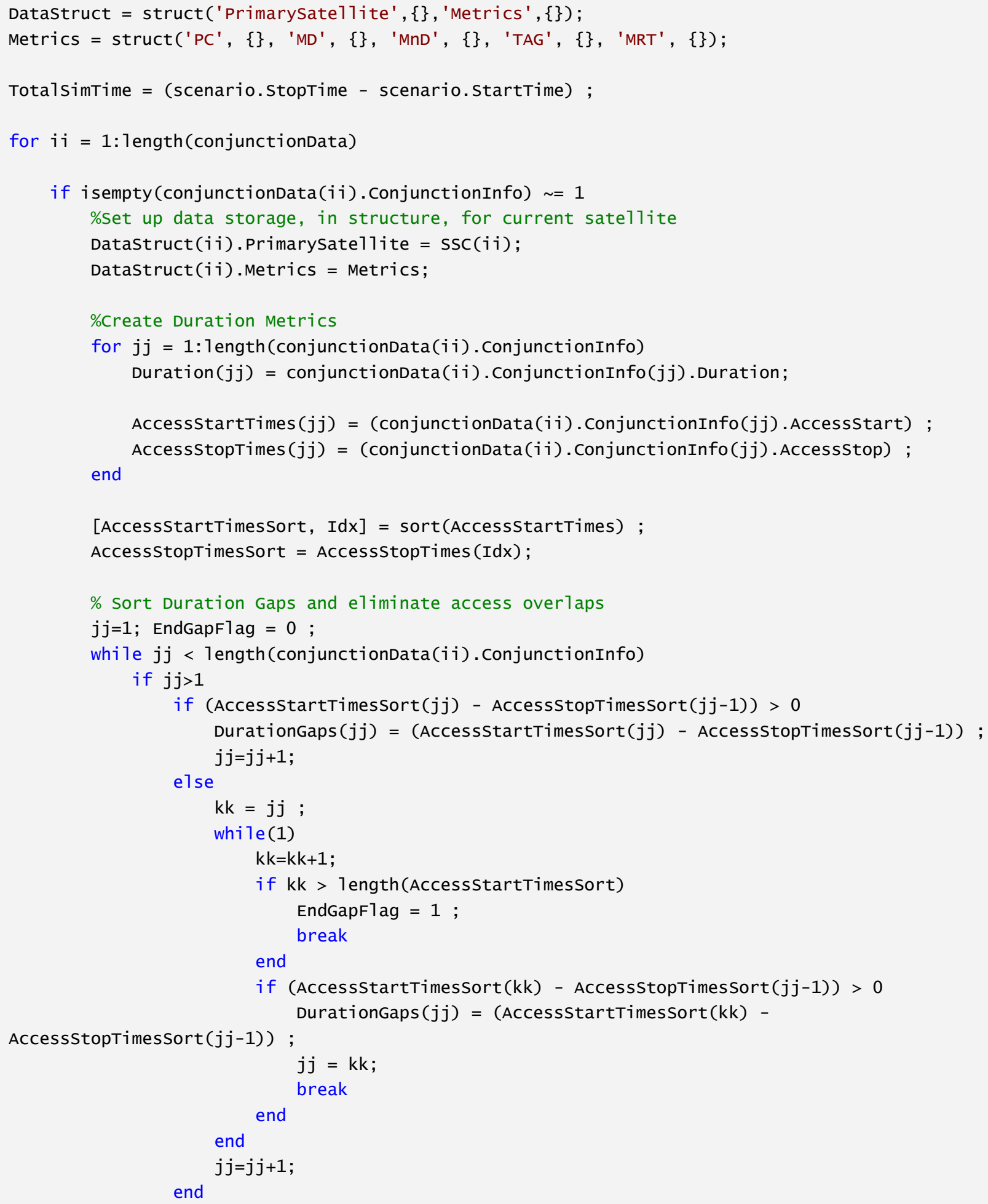




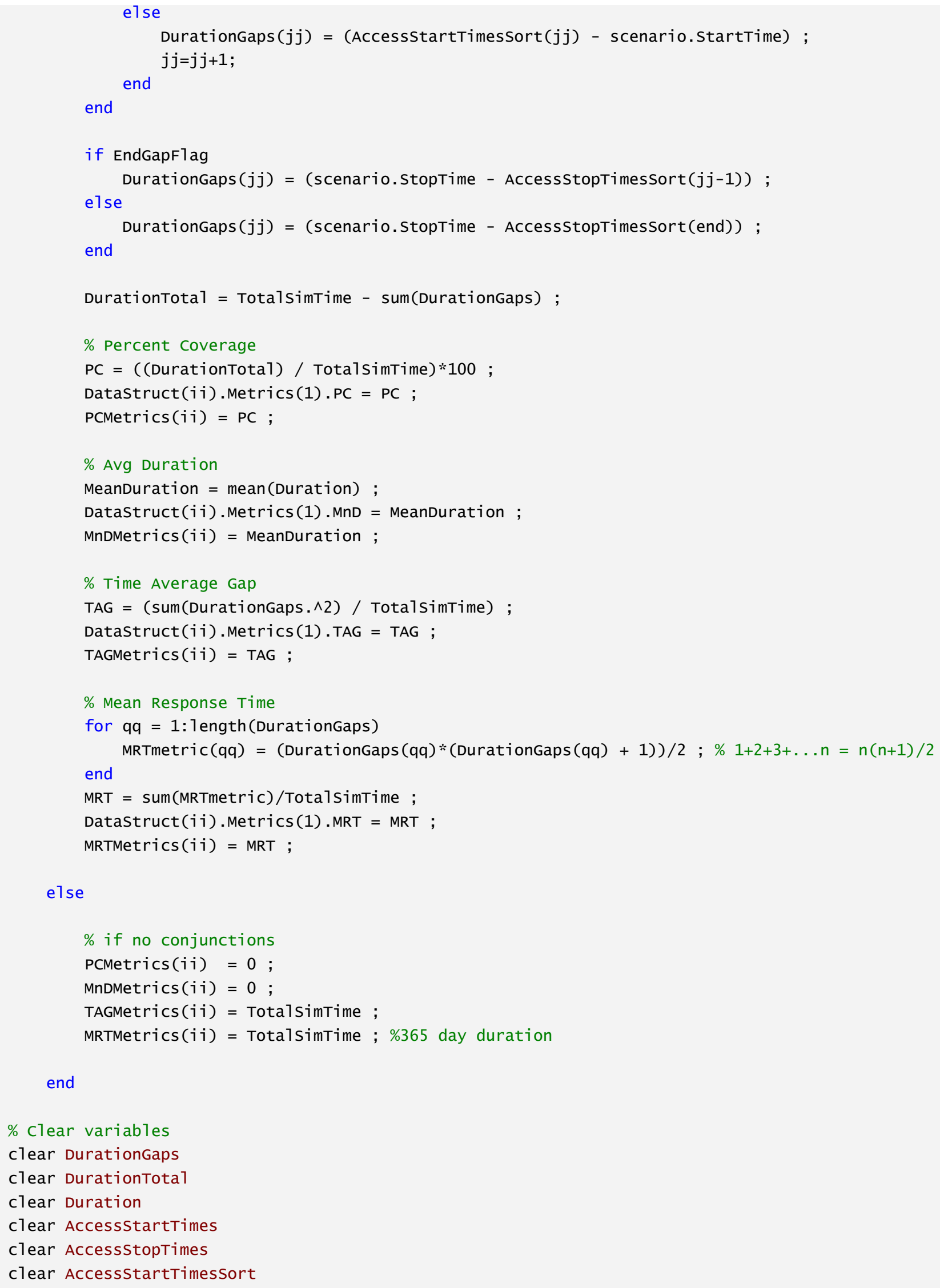




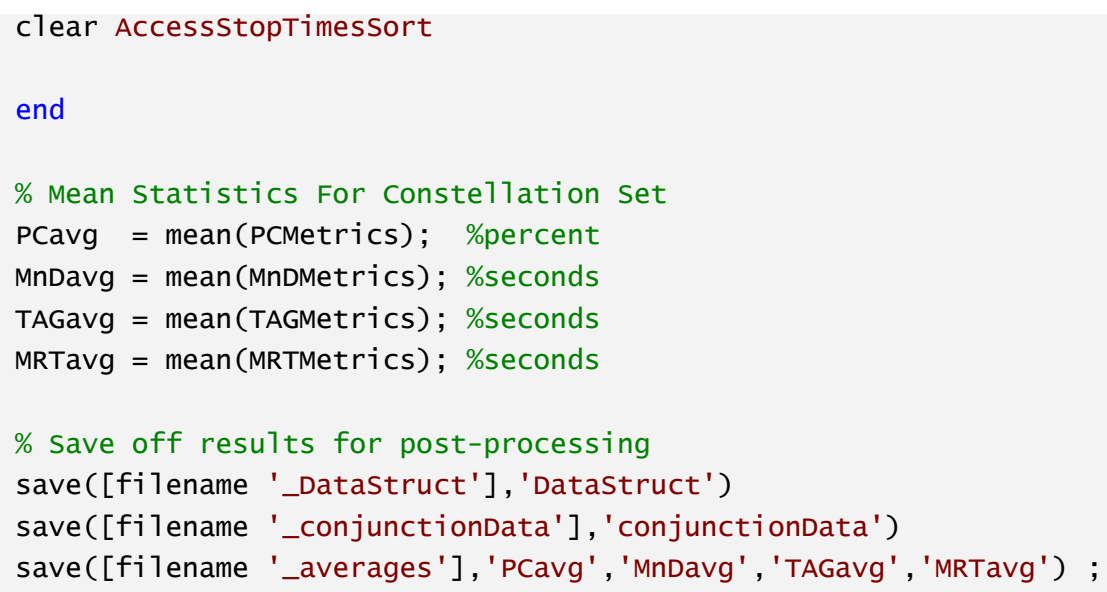

Published with MATLAB®R2016b 